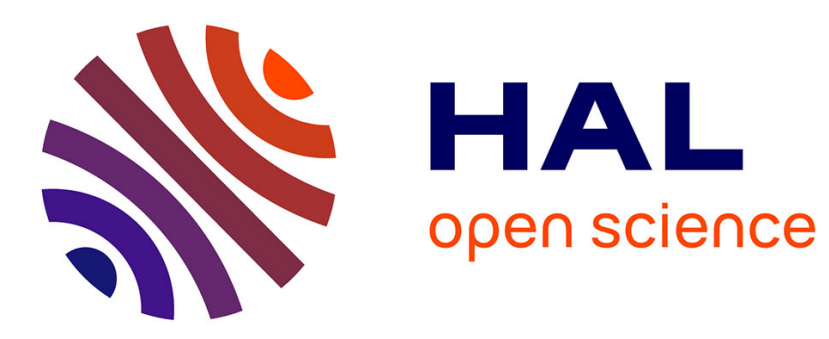

\title{
Modélisation et Statistique Spatiales
}

Xavier Guyon

\section{To cite this version:}

Xavier Guyon. Modélisation et Statistique Spatiales. École thématique. Modélisation et Statistique Spatiales (transparents ppt), Atelier RASMA Université Gaston Berger, Saint Louis du Sénégal, 2010, pp.223. cel-00762830

\section{HAL Id: cel-00762830 \\ https://cel.hal.science/cel-00762830}

Submitted on 8 Dec 2012

HAL is a multi-disciplinary open access archive for the deposit and dissemination of scientific research documents, whether they are published or not. The documents may come from teaching and research institutions in France or abroad, or from public or private research centers.
L'archive ouverte pluridisciplinaire HAL, est destinée au dépôt et à la diffusion de documents scientifiques de niveau recherche, publiés ou non, émanant des établissements d'enseignement et de recherche français ou étrangers, des laboratoires publics ou privés. 


\title{
Modélisation et Statistique Spatiale
}

\author{
Atelier RASMA - Université Gaston Berger \\ Saint Louis du Sénégal \\ 29 novembre - 4 décembre 2010 \\ Xavier Guyon - SAMM -- Université Paris 1
}

- Géostatistique, modèle du second ordre, krigeage

- Donnée sur un réseau :

Auto-Régression Spatiale (SAR et CAR, SARX)

Champ de Gibbs - Markov - Auto modèle de Besag

Simulation par chaîne de Markov (MCMC)

- Processus ponctuel 


\section{Trois types de structures spatiales}

\section{Géostatistiques :}

- S est un ensemble continu (de $\left.\mathrm{R}^{* * 2} 2, \mathrm{R} * * 3, \ldots\right)$

- données réelles, uni ou multidimensionnelles

- Observations en $\mathrm{n}$ sites : $\mathrm{s} 1, \mathrm{~s} 2, \ldots, \mathrm{sn}$ de $\mathrm{S}$.

\section{Latticielles :}

- S est un réseau fini de sites discret muni d'un graphe

- données réelles : modèles AR.

- ou non : champ de Markov (binaires, poisson, etc.)

\section{Données ponctuelles}

- S est un ensemble de points aléatoires $\mathrm{x} 1, \mathrm{x} 2, \ldots, \mathrm{x} n$ de $\mathrm{S}$, une partie de $\mathrm{R} * * 2$, $\mathrm{R}^{* *} 3, \ldots$ (Processus Ponctuel Spatial).

- PP marqué si une marque mi attaché à chaque xi : épicentre d'un séisme et mi $=$ intensité du séisme: $\{(\mathbf{x i}, \mathrm{mi}), \mathrm{i}=1, \mathrm{n}\}$. 


\section{Données géostatistiques $\mathrm{x}$}

(a) Cumul de pluies dans 100 stations météo suisse le jour du passage du nuage de Tchernobyl : réseau irrégulier (sic.100 de geoR)

(b) Porosité d'un sol (soil250 de geoR) : réseau régulier La dimension des symboles est proportionnelle à $X$
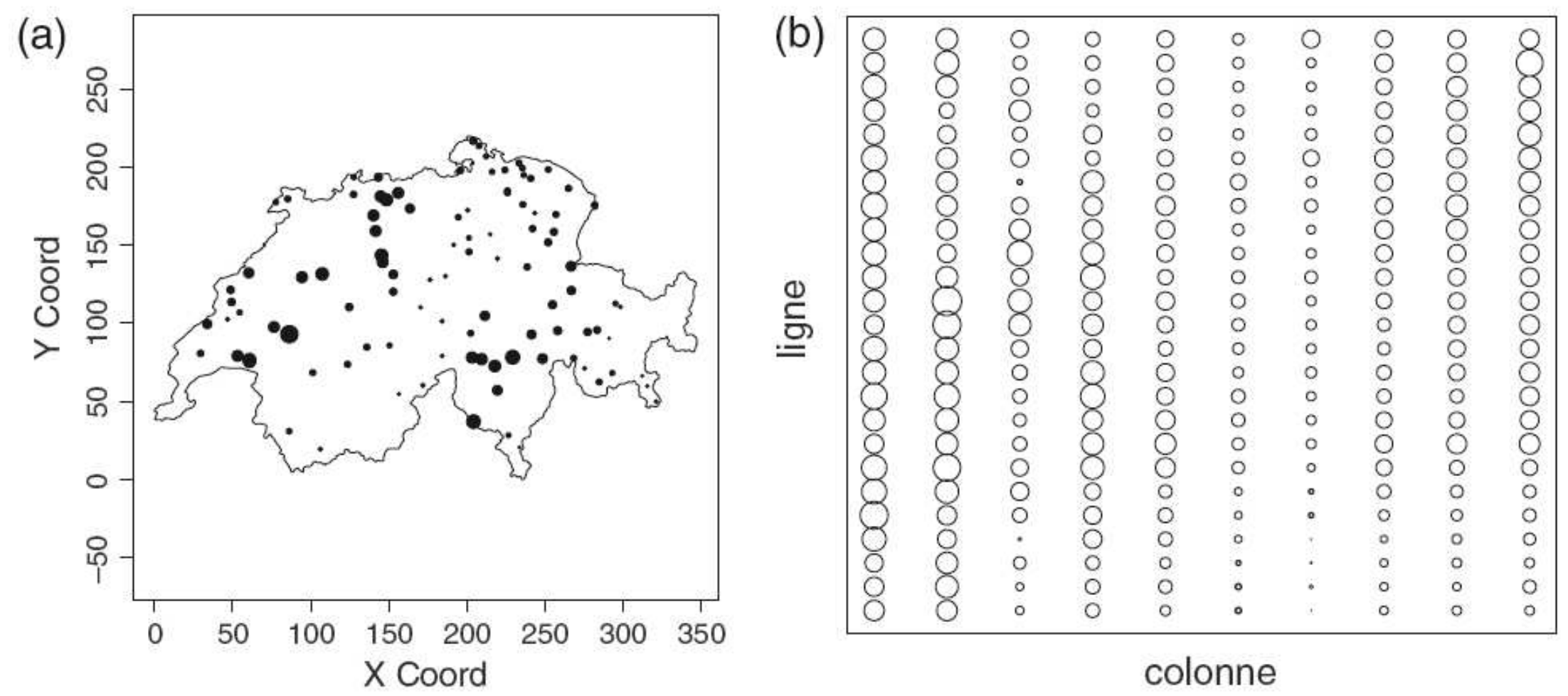

Atelier Spatial RASMA Saint Louis du Sénégal 


\section{Le logiciel $R$}

Installation de $R$ :

$$
\text { http://cran.r-project.org/ }
$$

- $\quad$ site miroir : i.e. Toulouse

- Deux fenêtres : R Console (RGui) et $\mathbf{R}$ Graphics

Chargement du package geoR (données géo-stat) 


\section{Données Porosité (soil250)}

22 variables « chimiques » sur une grille régulière $10 \times 25$ points espacés de 5 mètres (cf. soil 250 dans la liste de geoR). On sélectionne la coordonnées $n^{\circ} 16$, ctc (catium exchange)

$>\operatorname{data}($ soil250)

$>$ ctc <- as.geodata (soil250, data.col=16)

$>$ plot (ctc)

\section{4 graphiques}

1 - les 4 quartiles (4 couleurs) de CTC

2 et 3 - les nuages $(\operatorname{ctc}(x, y), y)$ et $(x, \operatorname{ctc}(x, y))$

4 - Histogramme de répartition des 250 valeurs de ctc

\section{Conservation d'un graphique :}

se placer dans la fenêtre graphique $\rightarrow$ historique $\rightarrow$ Ajouter (ou précédent, etc....)

Autre solution : placer la commande « > x11 () » avant une commande graphique conservera le graphique (aller dans fenêtre, les graphiques sont numérotés séquentiellement) 


\section{Données pluviométrie Suisse}

$>$ print (sic.100)

$>$ points(sic.100,borders=sic.borders)

sic.borders : fichier frontière

4 données pour chaque station : coordonnées $(x, y)$, hauteur de pluie, altitude

sic. 100 : 100 stations choisies au hasard dans un réseau de 367 stations

sic.all : toutes les 367 stations

$>$ points (sic.all, borders=sic.borders)

$>$ plot (sic.all) 

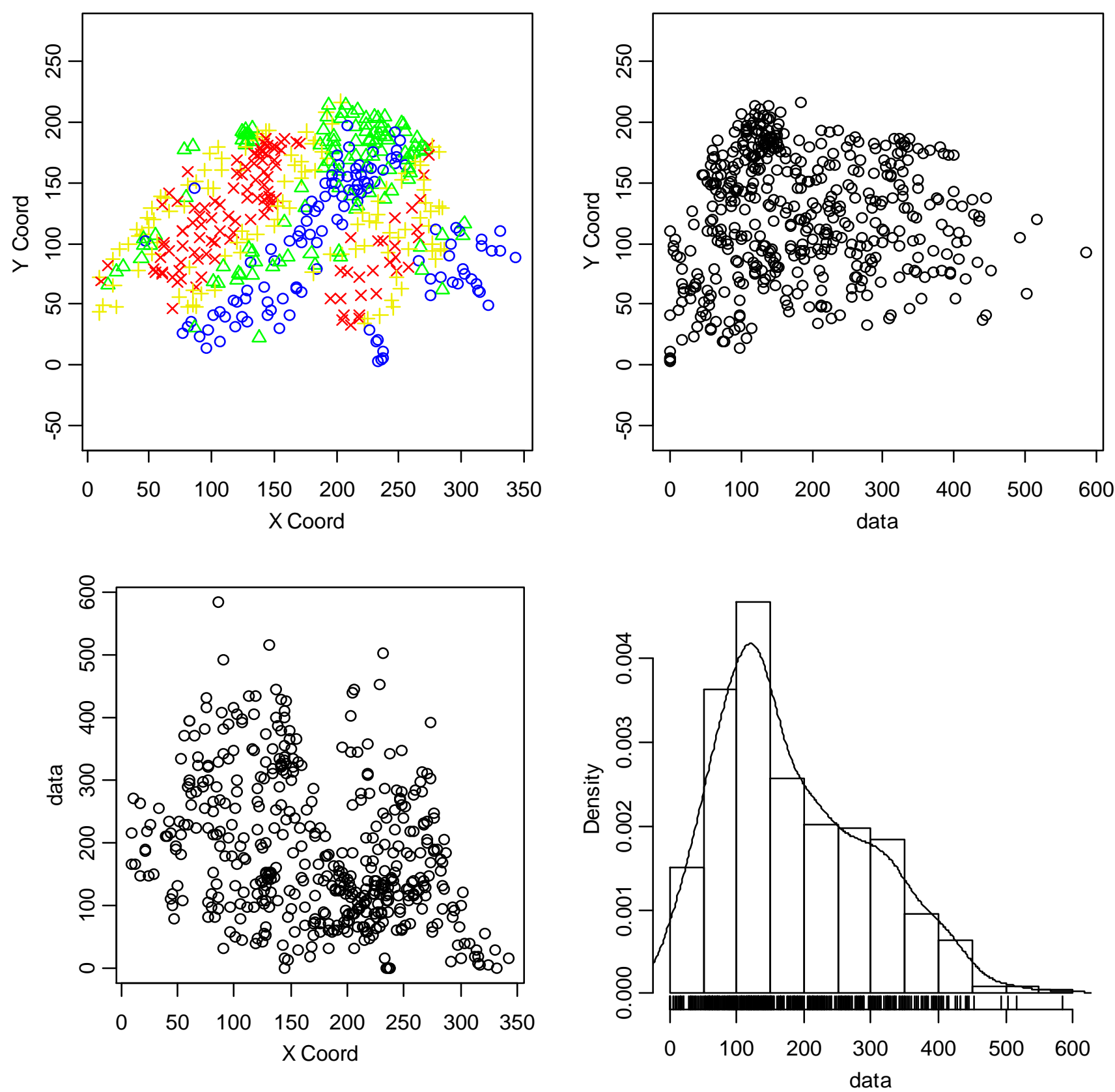

Atelier Spatial RASMA Saint Louis du Sénégal 


\section{Questions en Geostatistique}

- Quelle structure de corrélation spatiale ?

- Stationnarité (covariance), isotropie?

- Non stationnarité (variogramme)

- Modèle avec covariables (données exogènes)

- Estimation (validation) de modèle

- Prédiction partout : carte de krigeage, simulation conditionnelle

- Outil logiciel : geoR 


\section{Données réelles sur un réseau discret}

(a) \% groupe sanguin A dans 26 comtés Irlande (eire, spdep) (b) Image $256 \times 256$ de J. Lennon (193 niveaux de gris, lennon du package $f i e l d s$ )

$\rightarrow$ Packages: spdep, fields, ...

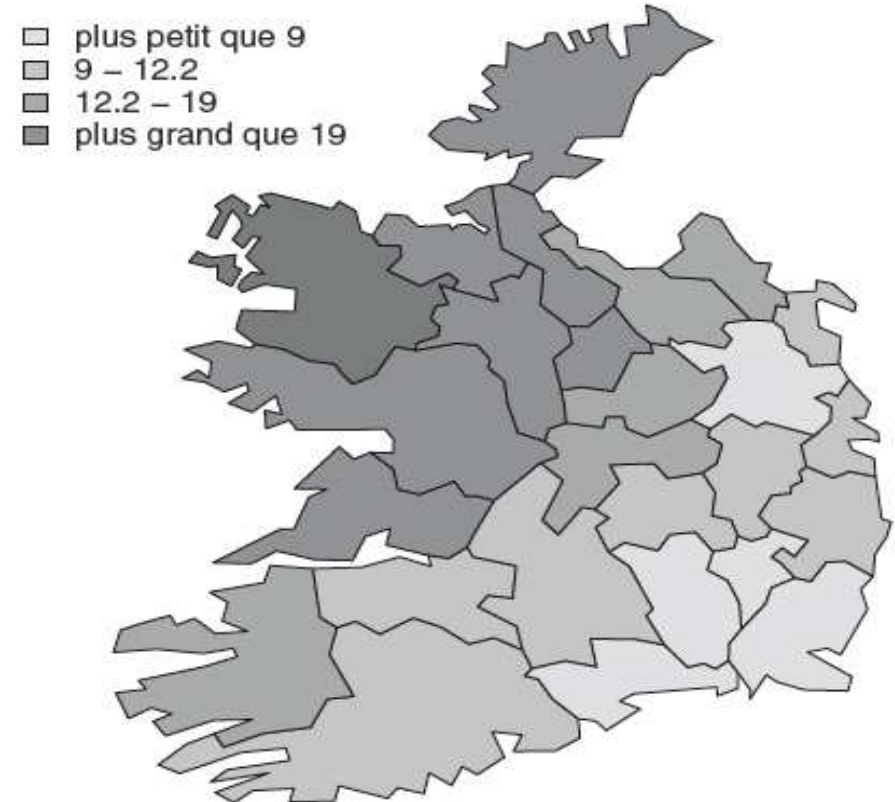

(a)

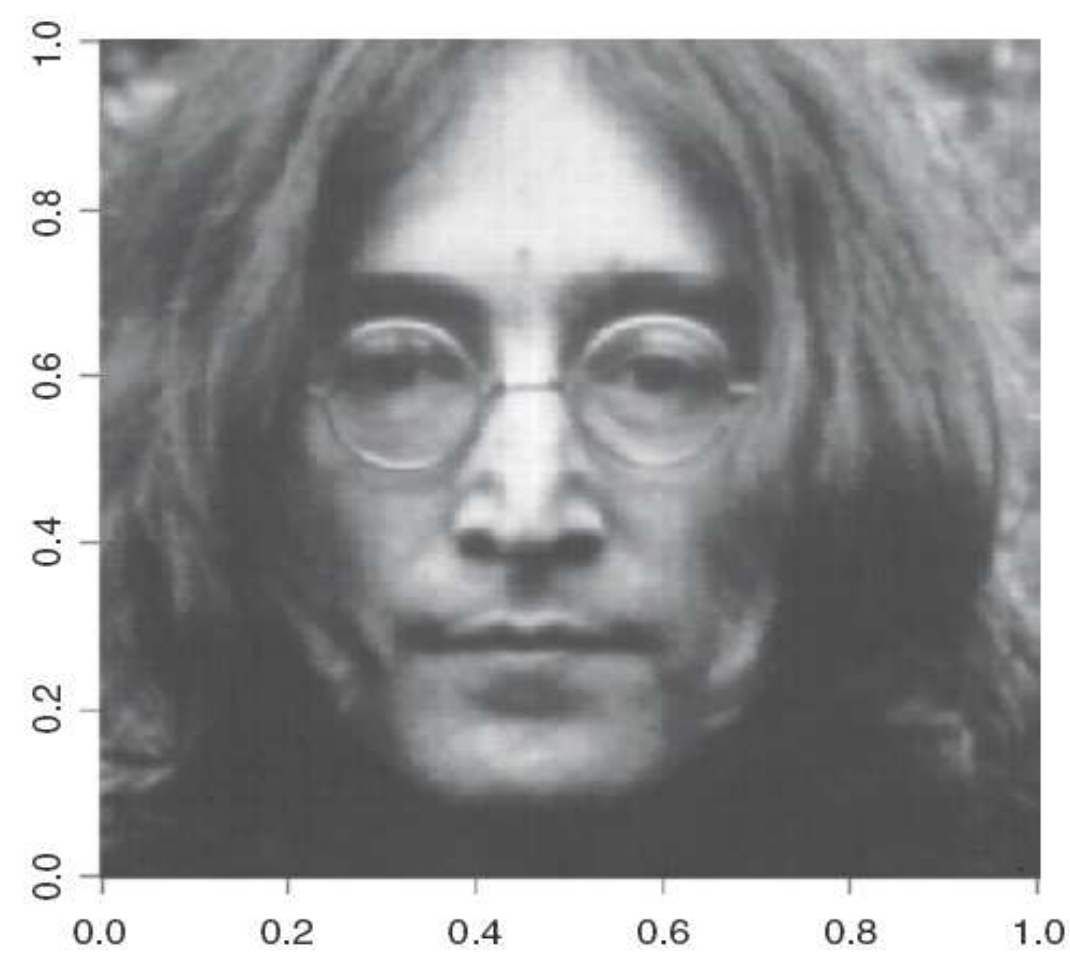

(b)

Atelier Spatial RASMA 
Pourcentage du groupe sanguin A dans les 26 comtés de l'Irlande (données eire de spdep)

$\mathrm{X}(\mathrm{s})=\%$ du groupe sanguin A dans 26 comtés.

Percentage with blood group A in Ere

- Graphe de contiguité spatiale,

- Indice de Moran : $\mathrm{t}=4.66$ (corrélation spatiale significative).

\section{Covariables}

- Towns : densité urbaine,

- Pale: indique si le comté était sous contrôle anglo normand ou non.

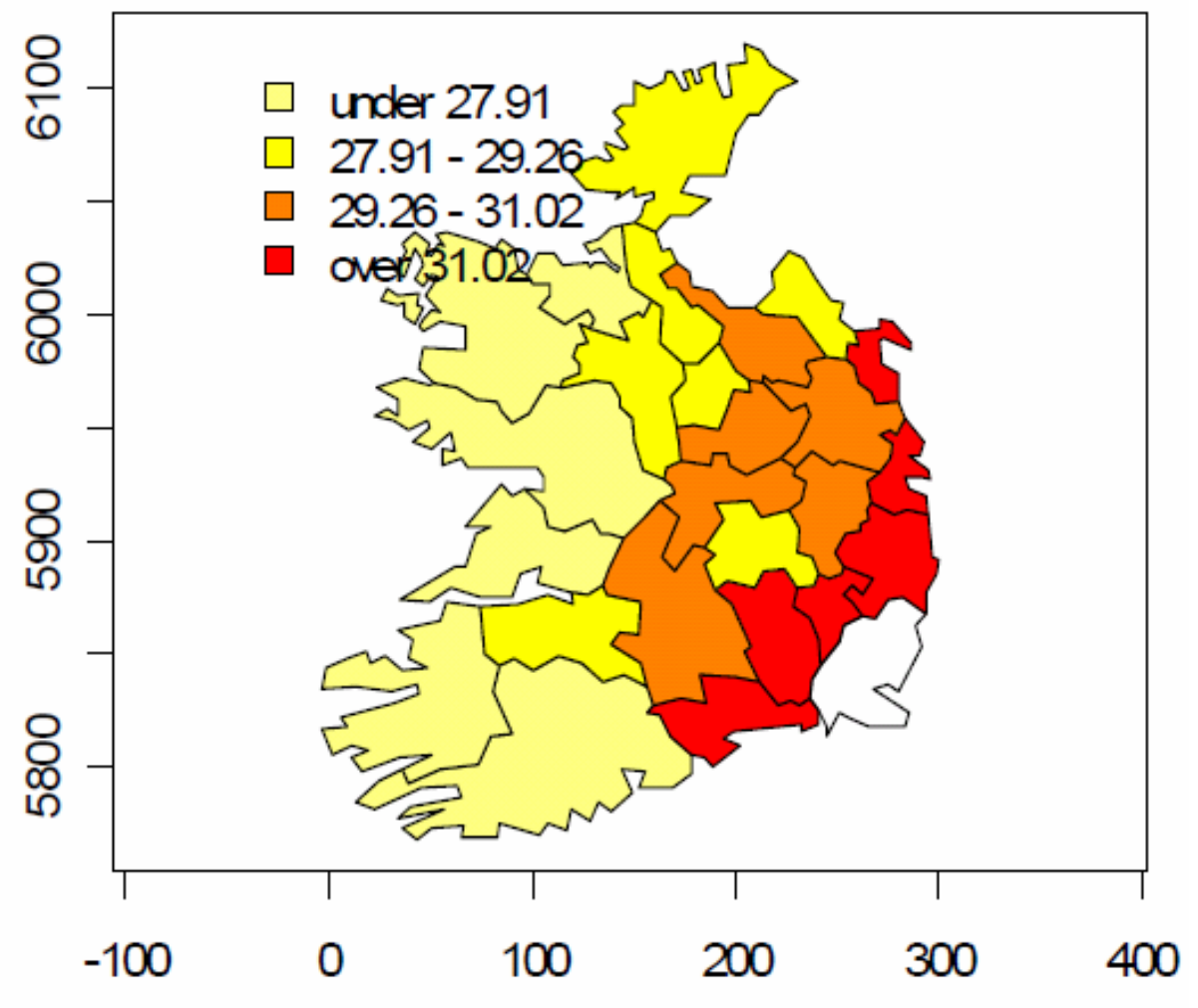


Données de Mercer et Hall : image en pixels de niveau de gris.

Image map of grain yield

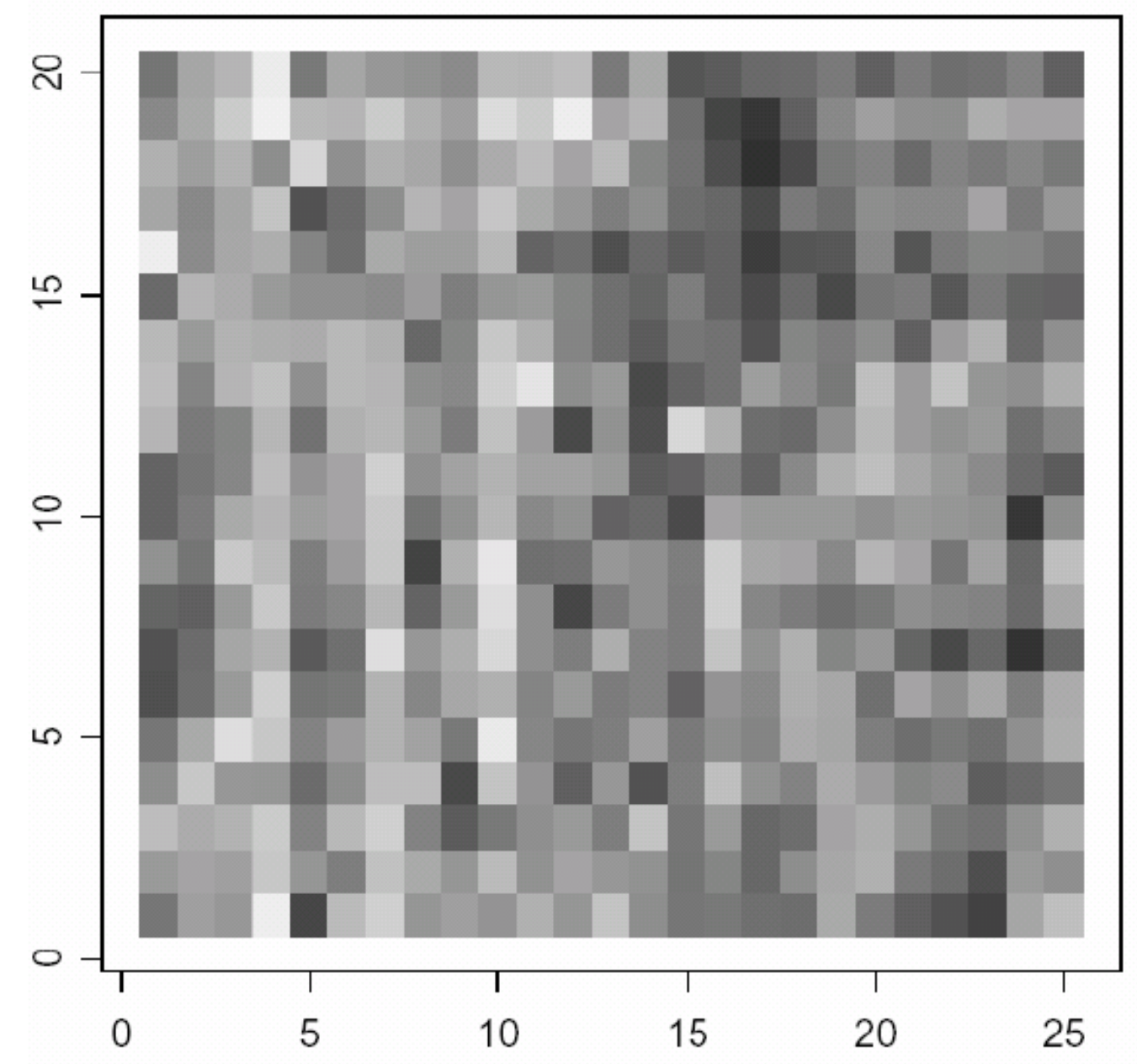

Atelier Spatial RASMA

Saint Louis du Sénégal 
Données latticielles : « la mort subite du nourrisson »

Nombre de cas dans 100 comtés de Caroline du nord entre 1974-

1978 (données sids de $\boldsymbol{R}$; Cressie,1993)

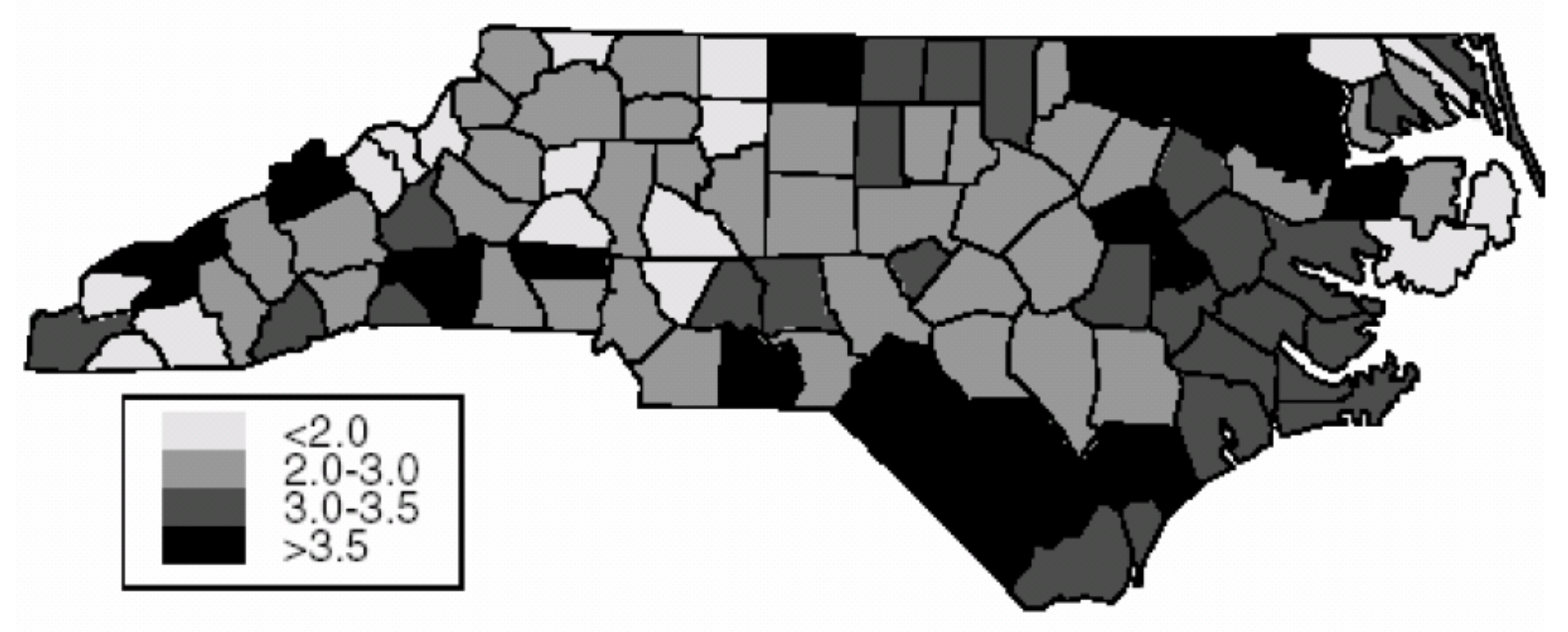




\section{Questions}

- Quel modèle ?

- voisinages d'influence pour chaque site

- SAR ou CAR

- stationnaire ou non

- avec variables exogènes (SARX)

- Estimation et validation de modèle

- Tests sur les paramètres ....

- Outils logiciel : spdep, fields, ... 


\section{Données SIDS : la mort subite du nourrisson (suite)}

Données : $\mathrm{X}(\mathrm{s})$ = taux de $\mathrm{msn}$ pour le canton s dans 100 cantons de north carolina, (1974-78), réelles.

Cantons et graphe de voisinage

Covariables (en s) :

- Taux $\mathrm{X}(\mathrm{ds})$ au voisinage $\mathrm{s}$,

- Nombre total de naissances,

- Pourcentage par communauté, etc

\section{Questions :}

- Auto corrélation spatiale?

- Modélisation régression spatiale, SAR, régression AR, SARX (avec eXogènes)....

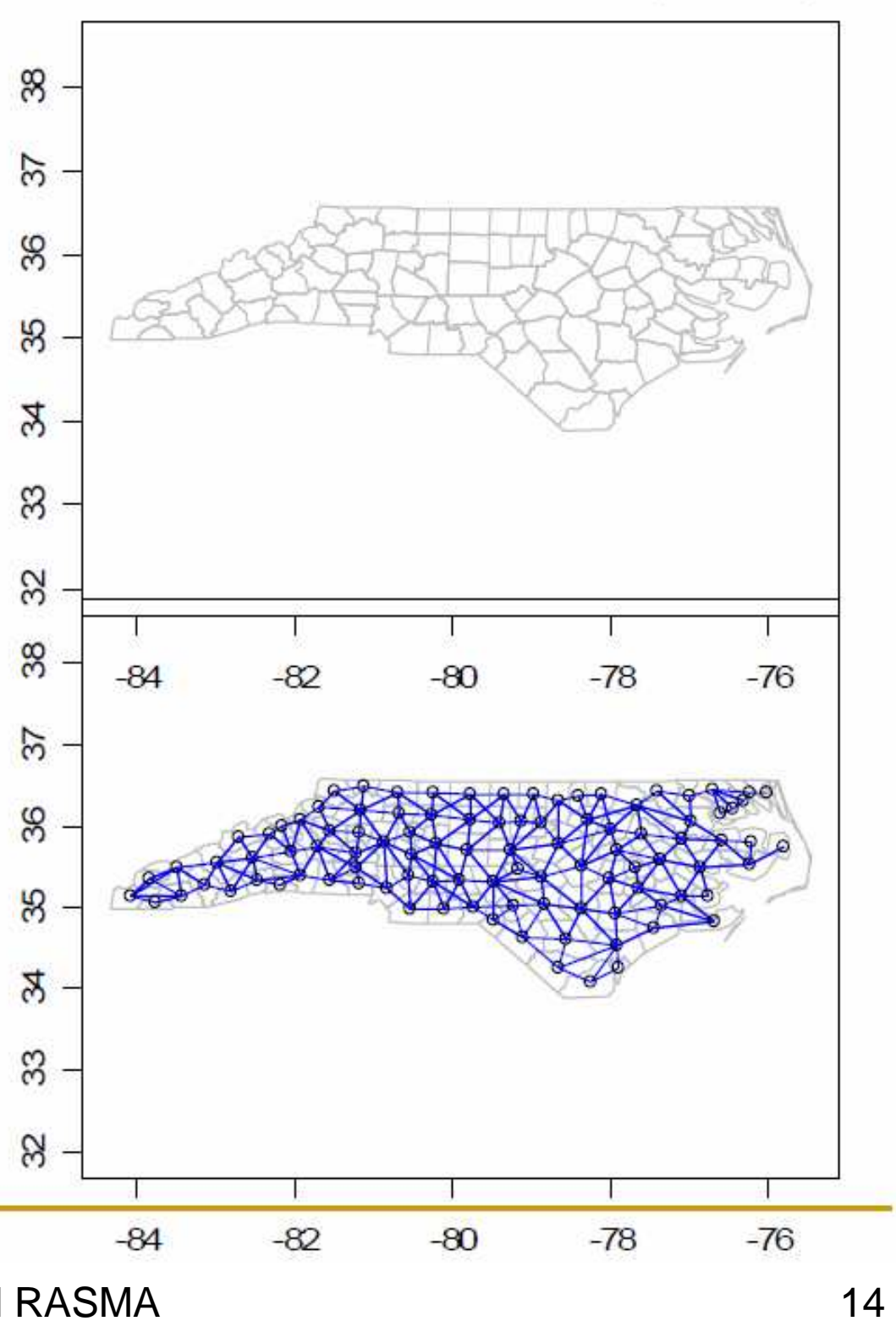




\section{Modèle de Gibbs - Markov}

Ex : répartition spatiale d'une espèce végétale

présence / absence de la grande laîche

Modèle de Auto - Logistique $\{0,1\}$

Voisinage de dépendance? Estimation, validation, tests ? Simulation

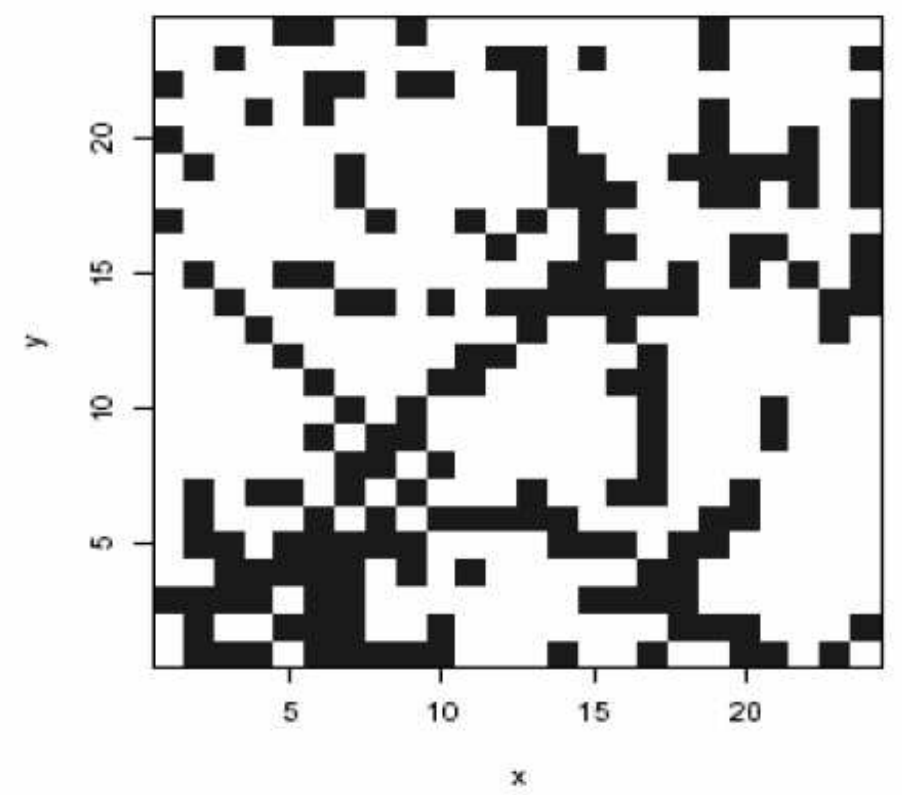

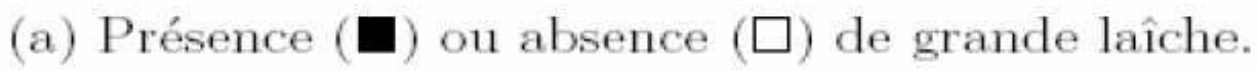




\section{Données Ponctuelles}

\section{$\boldsymbol{x}=$ configuration spatiale de $\boldsymbol{\Omega}$ points \\ 3 exemples}

(a) - 97 fourmilières : données ants de spatstat

(b) -42 centres de cellules d'une coupe histologique (cells)

(c) -- 126 pins d'une forêt finlandaise (finpines)

Package spatstat 


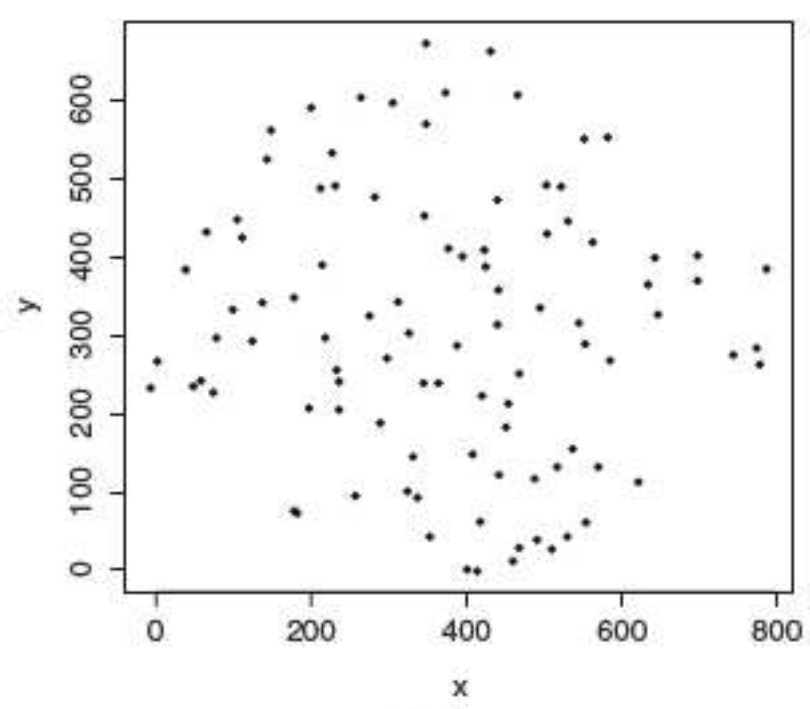

(a)

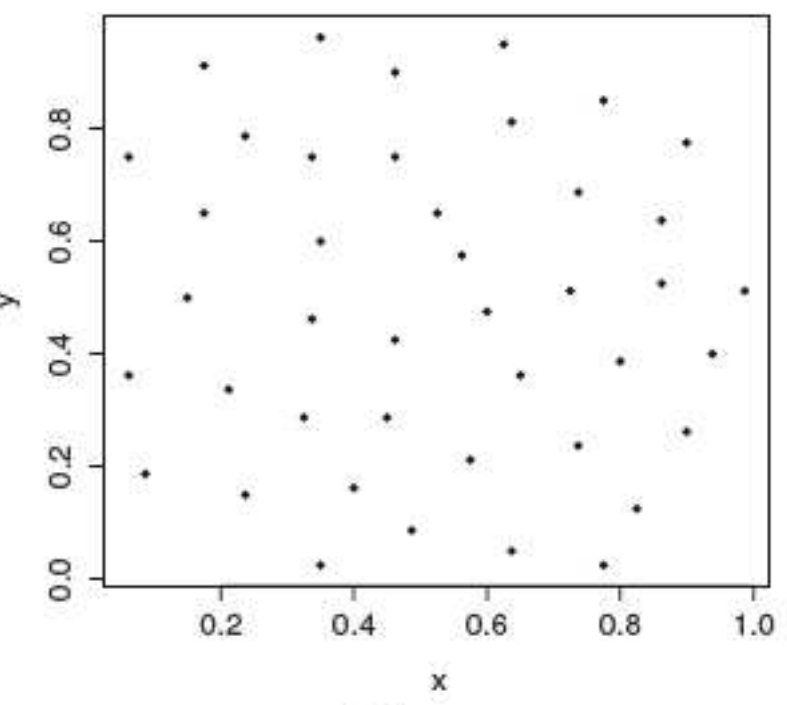

(b)

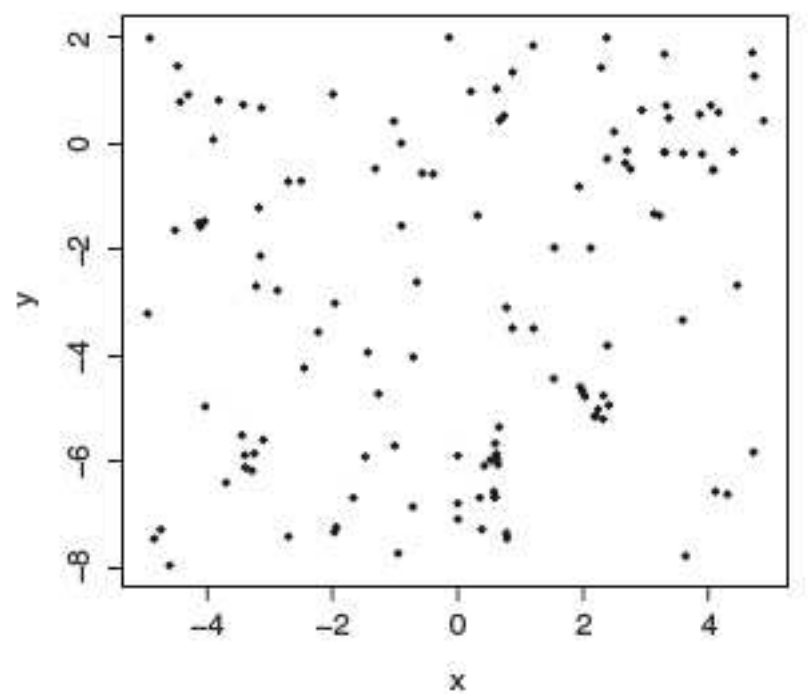

(c)

Fig. 3.1. Exemples de répartition ponctuelle : (a) 97 fourmilières (données ants du package spatstat); (b) 42 centres de cellules d'une section histologique observée au microscope (données cells de spatstat); (c) 126 pins d'une forêt finlandaise (données finpines de spatstat). 


\section{PP bivarié : 2 types de cellules de la rétine du lapin (294 en tout) \\ $>\operatorname{data(betacells)}$ et > plot (betacells)}

« on » $\left(^{\circ}\right)$ active à l'ouverture de la lumière, « off » (.) à la fermeture.

Question: ces cellules sont-elles sur une même couche?

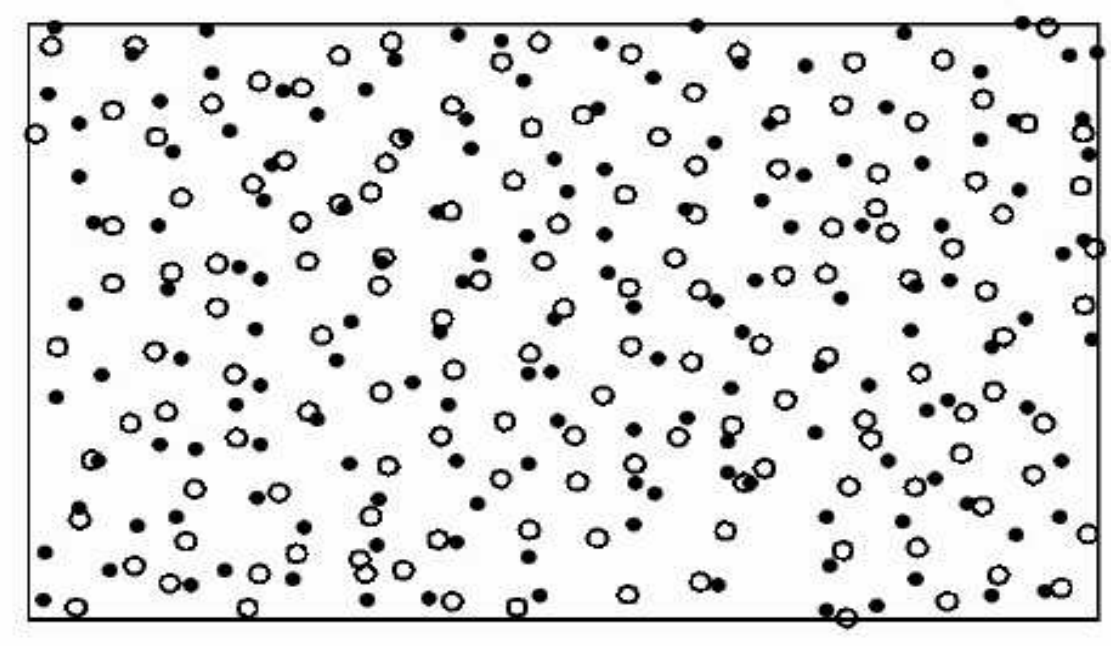




\section{Questions sur les Processus ponctuels}

- Répartition spatiale au hasard (P.P.de Poisson = CSR pour Complete Spatial Randomness))

- Ou non :

- avec compétition (chaque centre de cellule développe une zone d'influence)

- avec coopération (i.e. agrégats autour d'un père)

- Homogénéité spatiale ou non

- Quels modèles explicatifs?

- Statistique 


\section{Géostatistique \\ Modèles, prédiction, estimation}

Terminologie proposée par Matheron (1962, École des mines de Fontainebleau)

Initialement pour l'évaluation des réserves minières.

Aujourd'hui utilisée dans des domaines variés : environnement, épidémiologie, science de la terre, ....

Wackernagel (1995), Chiles et Delfiner (1999), Diggle et Ribeiro (2006). 


\section{Objectifs de la géostatistique}

\section{Modélisation}

- Modèle au second ordre, covariance, stationnarité

- Modèle intrinsèque :accroisements stationnaires

- Régularité : continuité, dérivabilité

- Prédiction à covariance connue : le Krigeage (simple, universel)

\section{Statistique}

- Nuée variographique

- Variogramme empirique

- Estimation d'un modèle paramétrique

- Validation de modèle: validation croisée, bootstrap paramétrique 


\section{Champ du second ordre $X$ sur $S\left(L^{* *} 2\right)$}

- Domaine d'étude : sites $s$ de $S$, sous ensemble de $R^{\star * 2}$

- Observation $X(s)$ réelle et de variance finie : $\operatorname{Var}(X(s))<\infty$

- $X$ caractérisé par ses lois finies dimensionnelles

$$
\begin{aligned}
& \text { Moyenne : } m(s)=E(X(s)) \\
& \text { Covariance }: c(s, t)=\operatorname{cov}(X(s), X(t))
\end{aligned}
$$

- Le plus souvent, modèle gaussien (pas une nécessité) 


\section{Différents Bruits Blancs (BB)}

- BB fort : variables $\{e(s)\}$ i.i.d.

- BB faible : variables centrées et de même variances

- BB gaussien : BB faible gaussien

- BB coloré : variables centrées même variances mais corrélées 


\section{Caractérisation d'une covariance : la semi définie positivité (sdp)}

$$
\begin{gathered}
\forall a \in \mathbb{R}^{m} \text { et } \forall\left(s_{1}, s_{2}, \ldots, s_{m}\right) \in S^{m}: \sum_{i=1}^{m} \sum_{j=1}^{m} a_{i} a_{j} c\left(s_{i}, s_{j}\right) \geq 0 \\
\operatorname{Var}\left(\sum_{i=1}^{m} a_{i} X_{s_{i}}\right)=\sum_{i=1}^{m} \sum_{j=1}^{m} a_{i} a_{j} c\left(s_{i}, s_{j}\right) \geq 0 .
\end{gathered}
$$




\section{Champ gaussien $X$}

Si toute combinaison linéaire est gaussienne

$X$ spécifié par sa moyenne $\mathrm{m}($.$) et sa covariance c(.,$.

$a=\left(a_{s}, s \in \Lambda\right), \sum_{s \in \Lambda} a_{s} X_{s}$ est une variable gaussienne.

$f_{\Lambda}\left(x_{\Lambda}\right)=(2 \pi)^{-\sharp \Lambda / 2}\left(\operatorname{det} \Sigma_{\Lambda}\right)^{-1 / 2} \exp \left\{-1 / 2^{t}\left(x_{\Lambda}-m_{\Lambda}\right) \Sigma_{\Lambda}^{-1}\left(x_{\Lambda}-m_{\Lambda}\right)\right\}$ 


\section{Champ stationnaire}

- Moyenne constante

- Covariance invariante par translation :

$$
c(s, t)=\operatorname{Cov}\left(X_{s}, X_{t}\right)=C(t-s)
$$

\section{Champ isotrope}

covariance invariante nar isotronie :

$$
c(s, t)=C_{0}(\|s-t\|)=C(s-t) .
$$




\section{Propriétés d'une covariance stationnaire $C$}

- C est semi-définie positive

- $I C(h) I \leq C(0)$

- $X(A s)$ est stationnaire si $s \rightarrow$ As est linéaire

- Une somme pondérée à coefficients $>0$ de covariances est encore une covariance

- Si $C$ est continue en 0 , alors $C$ est uniformément continue partout 


\section{Quelques covariances isotropiques Portée $a>0$ et Variance $\sigma^{* *} 2>0$}

- Pépitique: $C(0)=\sigma^{* *} 2$ et $C(h)=0$ sinon

- Exponentielle : $\mathrm{C}(\mathrm{h})=\sigma^{* *} 2 \exp (-a$ II $h$ II)

- Sphérique si $d \leq 3$

$$
\begin{aligned}
& C(h)=\sigma^{2}\left\{1.5\|h\| / a-0.5(\|h\| / a)^{3}\right\} \text { si }\|h\| \leq a \\
& C(h)=0 \text { sinon }
\end{aligned}
$$

- Gaussienne : $\quad C(h)=\sigma^{2} \exp \left(-(\|h\| / a)^{2}\right.$

(cf. liste assez complète dans > cov.spatial) 


\section{Modèle(s) de Matern}

- Plus un paramètre $v$ contrôle la régularité de $C$ en 0 (Kest la fonction de Bessel de première espèce)

- $v=1 / 2 \rightarrow$ cov. exponentielle

- $v=\infty \rightarrow$ cov. Gaussienne

- Plus $v$ augmente, plus $C(h)$ est régulière en 0 et plus $X$ est régulier (en moyenne quadratique)

$$
C(h)=\sigma^{2} 2^{1-\nu}(\|h\| / a)^{\nu} \mathcal{K}_{\nu}(\|h\| / a) / \Gamma(\nu)
$$




\section{Champ intrinsèque et variogramme}

- Considérer le champ des h-accroissements :

$$
X_{s+h}-X_{s}: s \in S
$$

- X intrinsèque si ses h-accroissements sont stationnaires

- Variogramme en h :

$$
2 \gamma(h)=\operatorname{Var}\left(X_{s+h}-X_{s}\right)
$$




\section{Stationnaire ou intrinsèque ?}

- Stationnaire $\rightarrow$ intrinsèque : $2 \gamma(h)=2(C(0)-C(h))$

- Intrinsèque $\neq>$ stationnaire :

Exemple : le mouvement brownien, $y(h)=|\mathrm{h}|$

- Un variogramme n'est pas toujours borné :

$$
\gamma(h ; b, c)=b\|h\|^{c}, 0<c \leq 2 .
$$

Exemple : vario puissance et auto-similarité ( $c=1$ pour le mouvement brownien). 


\section{Portée, palier, effet pépite d'un variogramme}

(a) Les 3 caractéristiques d'un variogramme

(b) Variog. expo.(1), sphérique (2) et gaussien (3) : régularité en 0 linéaire pour (1-2) et parabolique pour (3)

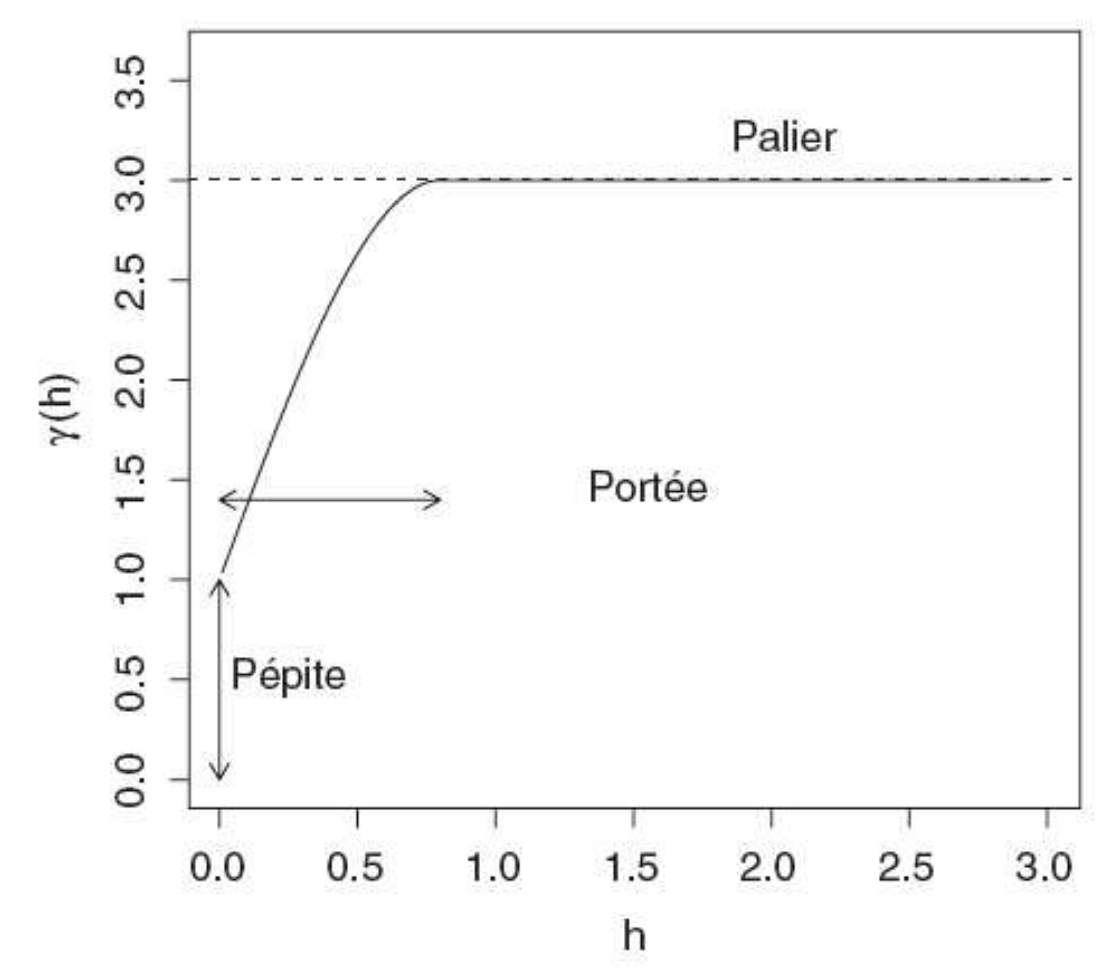

(a)

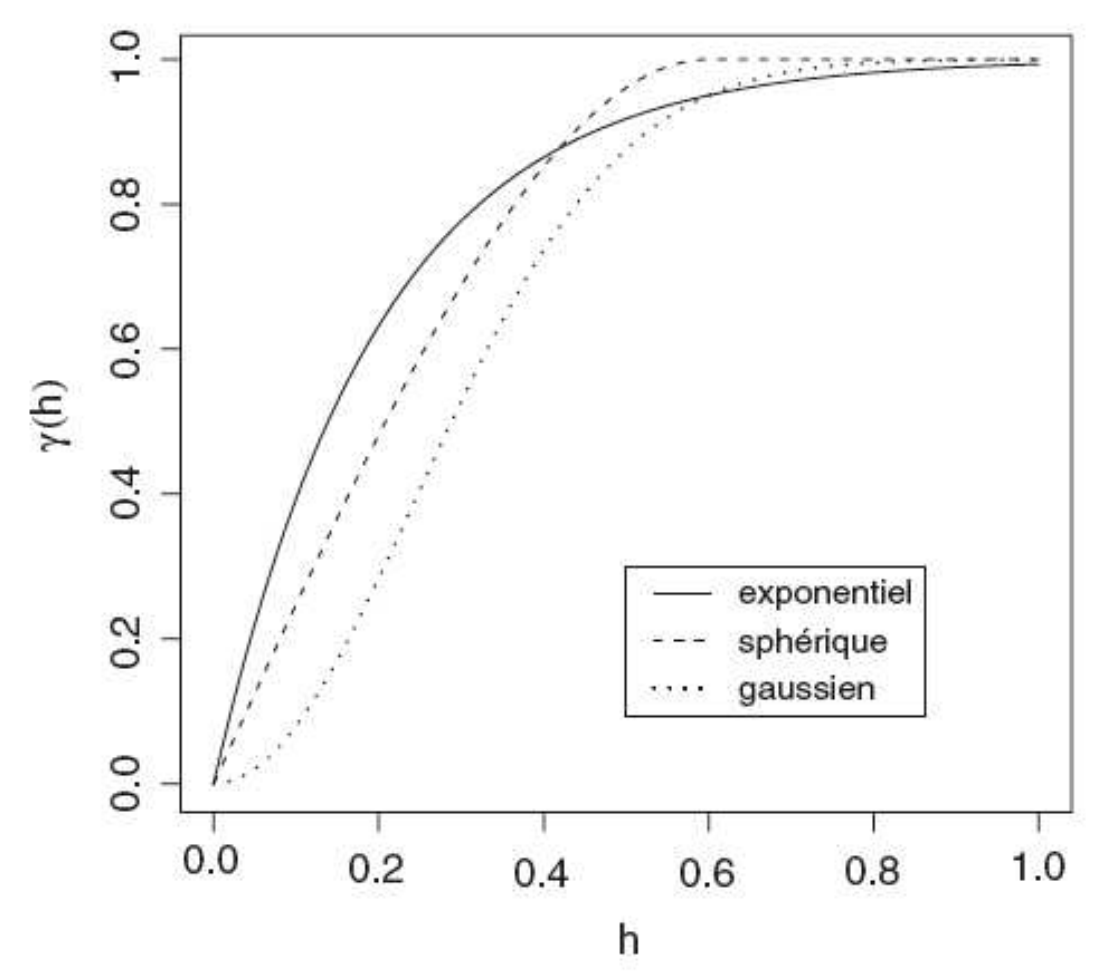

(b) 


\section{Régularité du variogramme de Matern}

- $v=1 / 2$ donne le variogramme exponentiel

- $\quad v \uparrow$, plus de régularité en 0

- $\quad v \geq 2, C$ dérivable en 0 à dérivée nulle

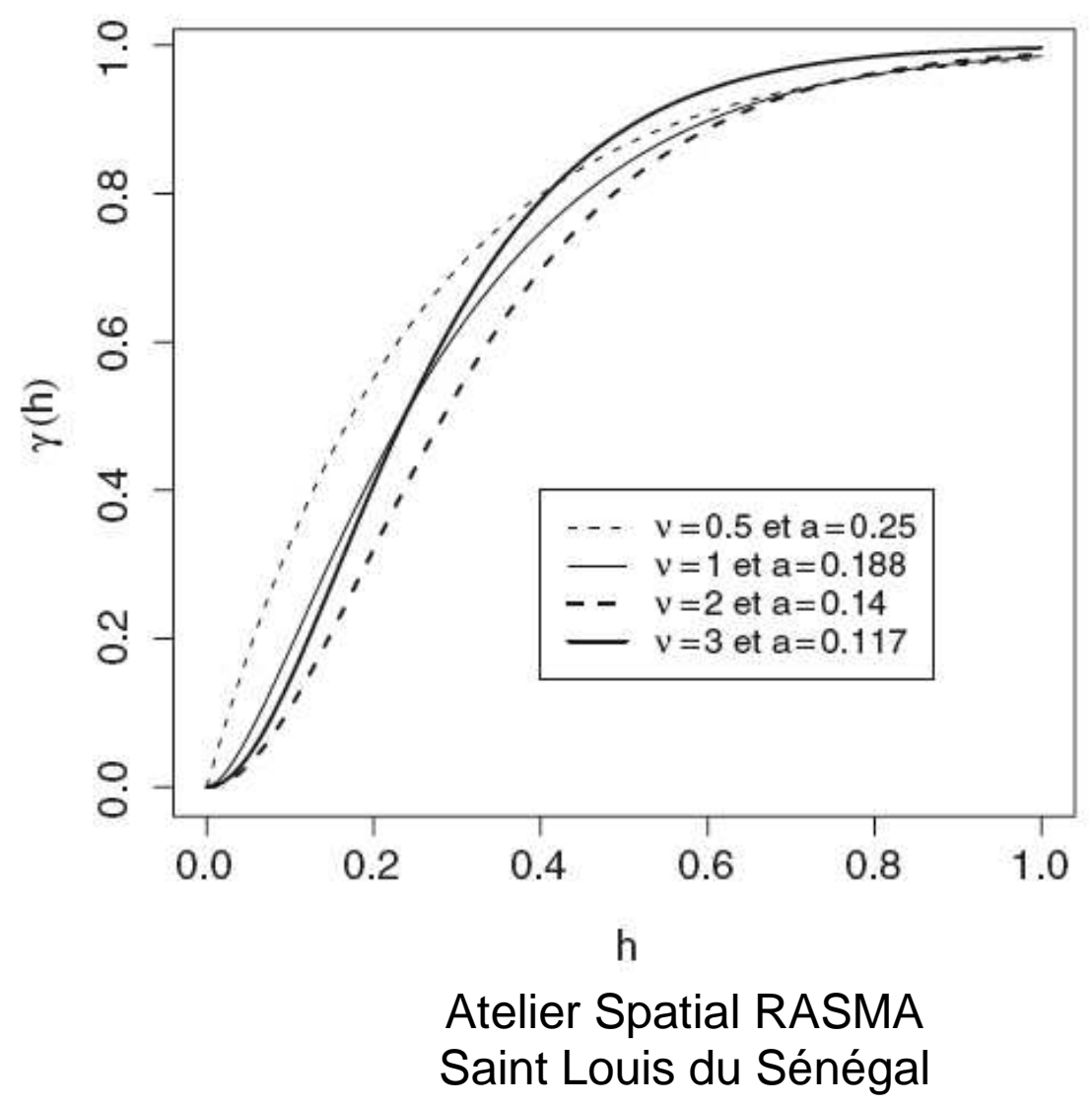


Cov.spatial de $R \rightarrow$ principales covariances spatiales Exemple : expo., sphérique, gauss de même portée pratique.

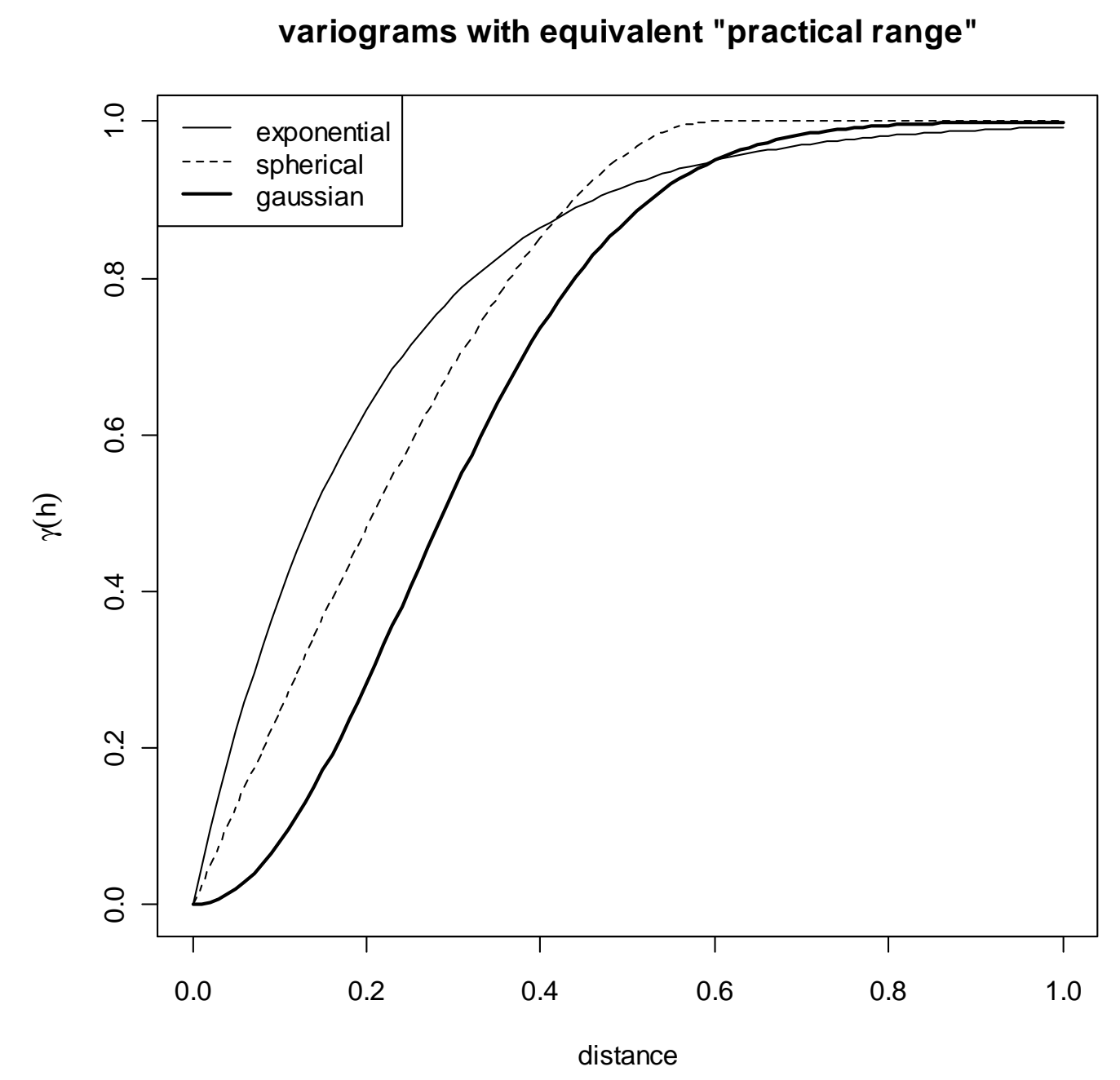

Atelier Spatial RASMA Saint Louis du Sénégal 


\section{Anisotropies}

Variogrammes différents suivant les directions

- Anisotropie géométrique : $\quad \gamma(h)=\gamma_{0}(\|A h\|)$

- Anisotropie zonale: $\gamma(h)=\gamma_{1}\left(\sqrt{h_{1}^{2}+h_{2}^{2}}\right)+\gamma_{2}\left(\left|h_{2}\right|\right)$ 


\section{Anisotropies}

(a) : géométrique

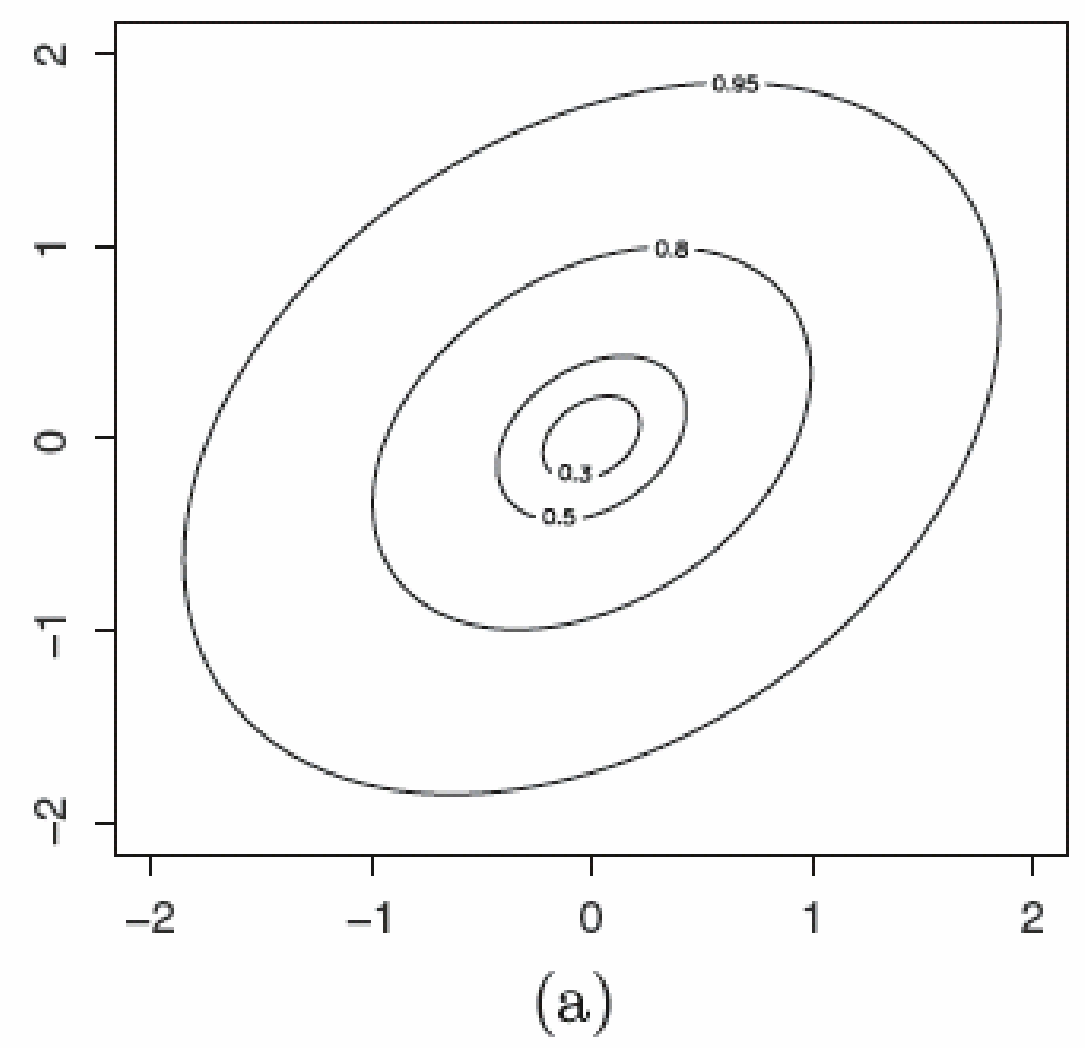

(b) zonale

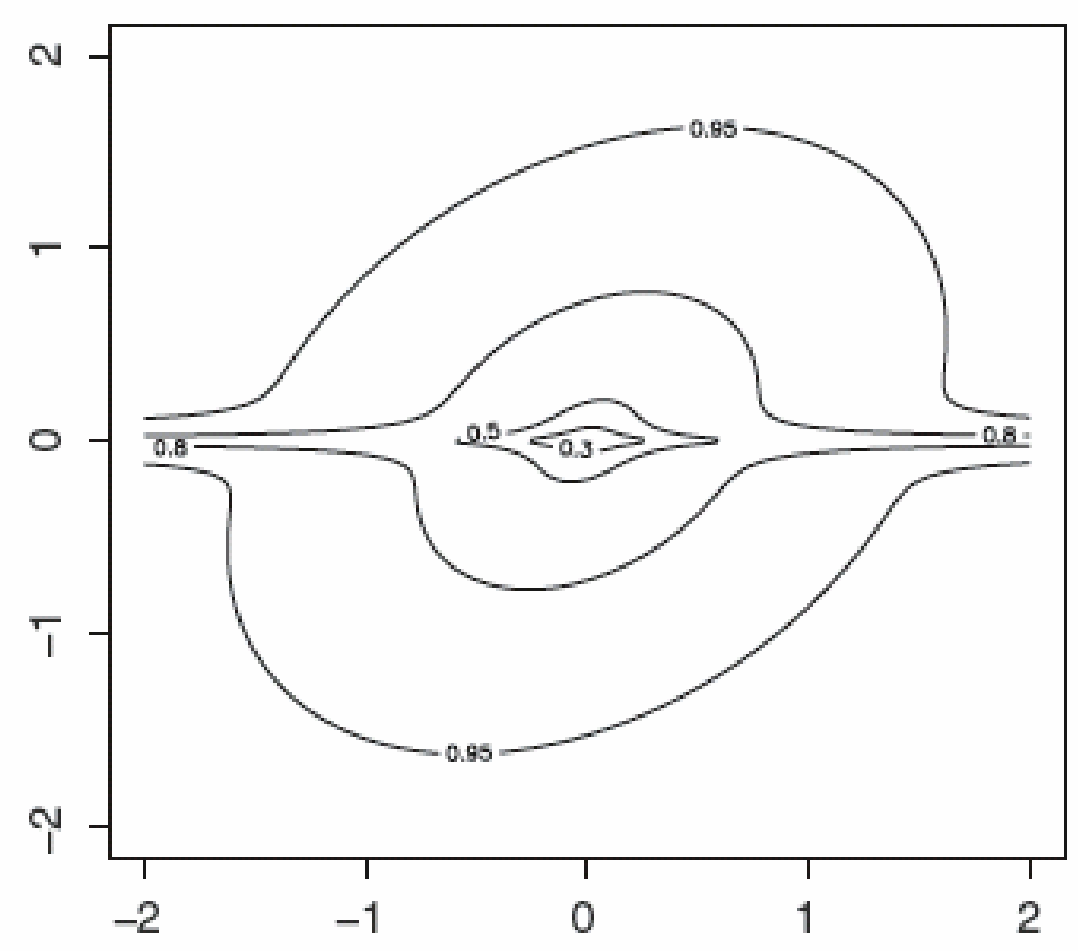

(b) 


\section{La régulatité de la covariance $C$ en 0 règle la régularité en m.q. de $X$ partout}

- $C$ continue en $0 \rightarrow X$ continu partout

- $C$ " exite en $O \rightarrow X$ dérivable partout

- Idem en remplaçant $C$ par le variogramme $y$

- Importance : régularité d'une carte de prédiction (Krigeage) est fonction du choix de $C$ (ou $\gamma$ ) 
Covariance « exponentielle de puissance » $\mathrm{C}(\mathrm{h})=\exp -(\mathrm{h} / \mathrm{phi})^{* *} \mathrm{k}$.

$\mathrm{C}(\mathrm{h})$ et simulations sur un intervalle pour $\mathrm{phi}=0.2$ et $\mathrm{k}=1$ (solide), 1.5 (tirets) et 2 (pointillés). La régularité en $0 \mathrm{de} h \rightarrow \mathrm{C}(\mathrm{h})$ augmente avec phi.

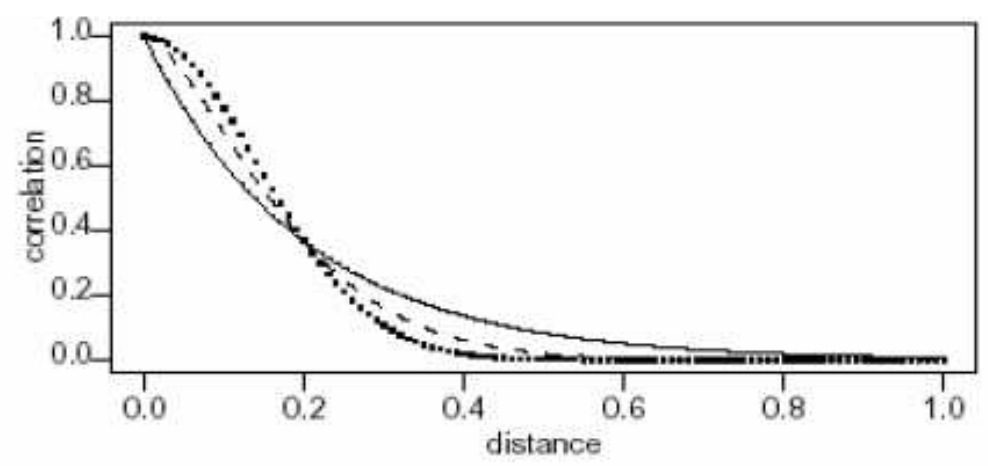

Figure 3.3. Throe examples of the powenod exponential cornelation function with $\phi=0.2$ and $\kappa=1$ (solid line), $\kappa=1.5$ (dashexd line) and $\kappa=2$ (dotted line).

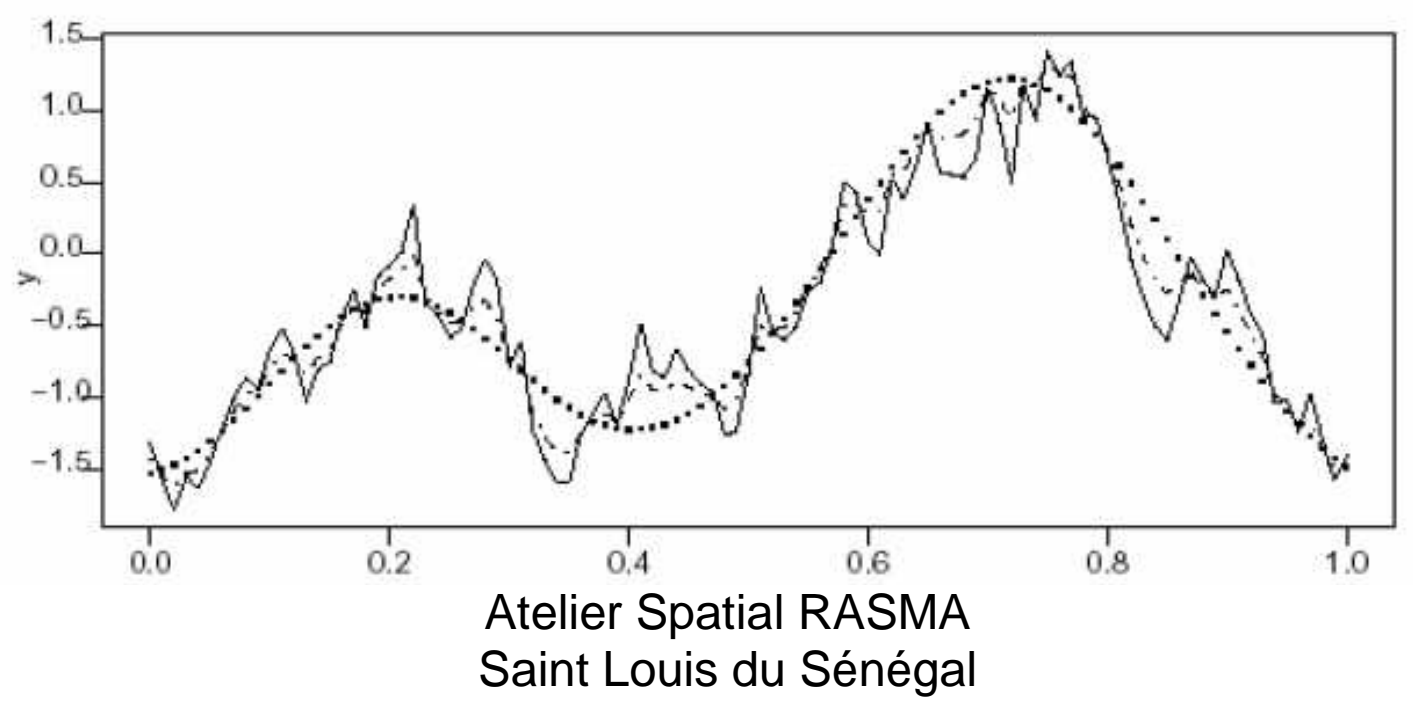




\title{
Simulation d'un champ gaussien
}

\author{
le package Randomfields
}

Gaus.SRF : simule un champ spatial ou spatio-temporel stationnaire Il faut déclarer :

la fonction de covariance

la grille de simulation

la tendance si il y en a une

la méthode de simulation retenue

(cf. $1^{\mathrm{er}}$ exemple de champ stable pour différentes grilles)

CovarianceFct : donne liste des covariances/variogramme

spatiaux ou spatio-temporel

CondSimu : réalise la simulation conditionnelle d'un champ gaussien en dehors des sites d'observation

ShowModels : démonstration interactive de simulation de modèles 


\section{Réalisations d'un champ gaussien isotropique}

à gauche pépitique (discontinu);

à droite exponentiel (continu mais non dérivable).
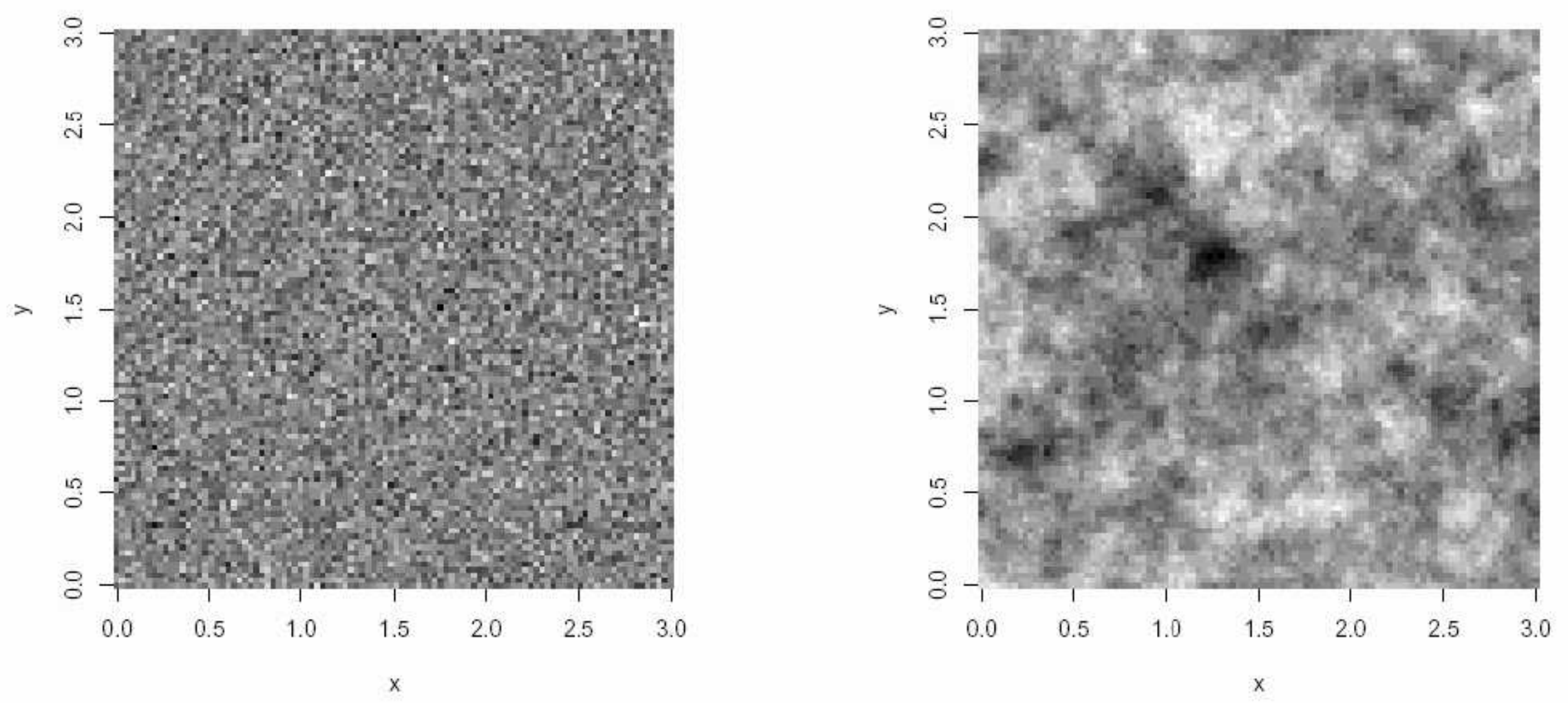


\section{(suite) Champ gaussien isotropique à variogramme gaussien}

à gauche, isotropique; à droite, anisotropique.

La surface $\mathrm{s} \rightarrow \mathrm{X}(\mathrm{s})$ est dérivable.
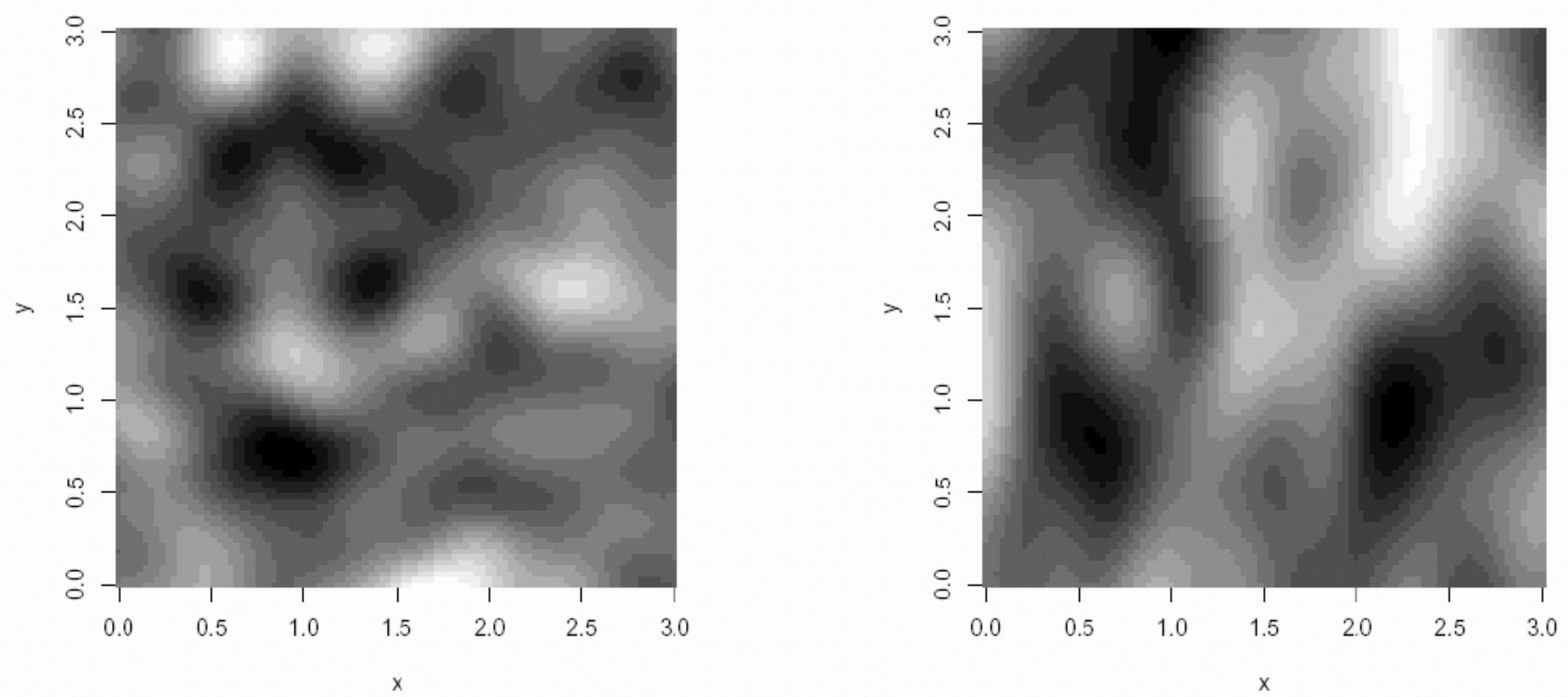


\section{Prédiction à moyenne et covariance connue : le Krigeage simple}

- Vecteur des $n$ observations $X=(X(s(1)), X(s(2)), \ldots, X(s(n)))$

- Prédire $X(s(0))$ (carte de krigeage) partout sur $S$

- Choix d'une covariance connue (variogramme) $C$ :

$$
\Sigma=\operatorname{cov}(X) \text { et } c=\operatorname{cov}(X(s(0)), X)
$$

- Reconstruction par MCO : minimiser

$$
\operatorname{EQM}\left(s_{0}\right)=E\left\{\left(X_{0}-\widehat{X}_{0}\right)^{2}\right\} .
$$

- si $X$ de moyenne $m$ connue, krigeage simple en $s(0)$

Prévision BLUP et Variance de prédiction :

$$
\widehat{X}_{0}={ }^{t} c \Sigma^{-1} X, \quad \tau^{2}\left(s_{0}\right)=\sigma_{0}^{2}-{ }^{t} c \Sigma^{-1} c .
$$




\section{Krigeage $=$ Prédiction $=$ Projection orthogonale pour le produit scalaire de la covariance}

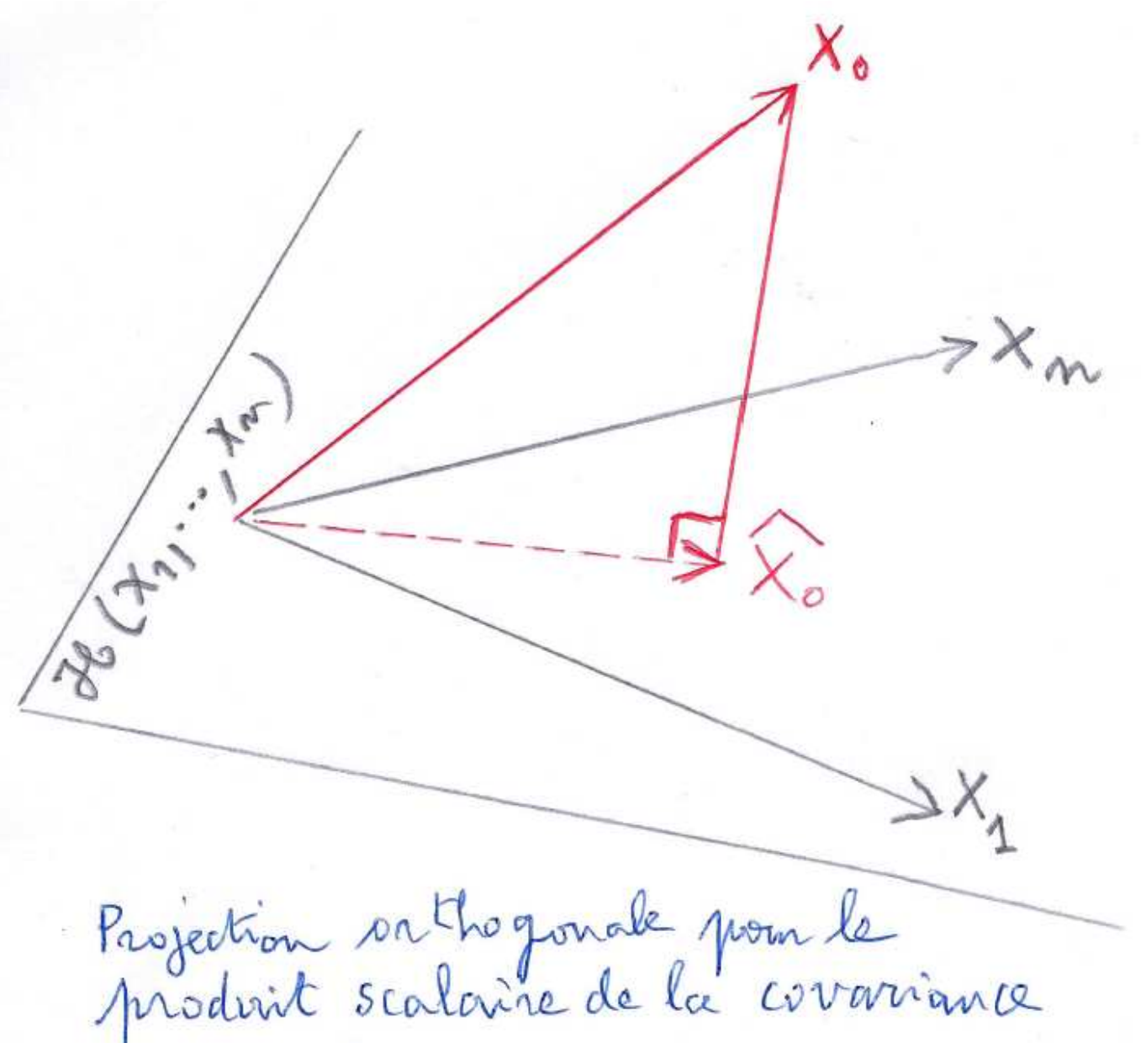




\section{Le Krigeage universel : $m$ inconnue}

- $\quad X$ suit un modèle de régression : covariables $Z$, paramètre $\delta$ inconnu, $\varepsilon$ résidu de covariance $\Sigma$

- Le krigeage :

$$
X=Z \delta+\varepsilon
$$

1. Estimer $\delta$ par $M C G$

2. Krigeage simple sur résidu $\varepsilon$

$$
\begin{aligned}
\widehat{X}_{0} & ={ }^{t} z_{0} \widehat{\delta}+c \Sigma^{-1}(X-Z \widehat{\delta}), \quad \text { avec } \\
\widehat{\delta} & =\left({ }^{t} Z \Sigma^{-1} Z\right)^{-1 t} Z \Sigma^{-1} X .
\end{aligned}
$$




\section{Krigeage / prédiction avec geoR}

- krige.conv

Effectue la prédiction partout (en fait sur une grille à définir) pour un modèle de variogramme donné (ou un modèle estimé par variofit ou par likfit)

Option pour le krigeage simple, ordinaire opu universel.

- Output.control

Réalise des simulations conditionnelles; permet d'évaluer la carte des probabilités de dépasser un seuil.

- Voir également les packages

$$
\begin{aligned}
& \text { Fields (Krig et sim.Krig.grid) et } \\
& \text { RandomFields (Kriging et CondSim) }
\end{aligned}
$$


Données de pluies Parana : codage en (taille/gris) associé aux quartiles empiriques de la distribution observée.

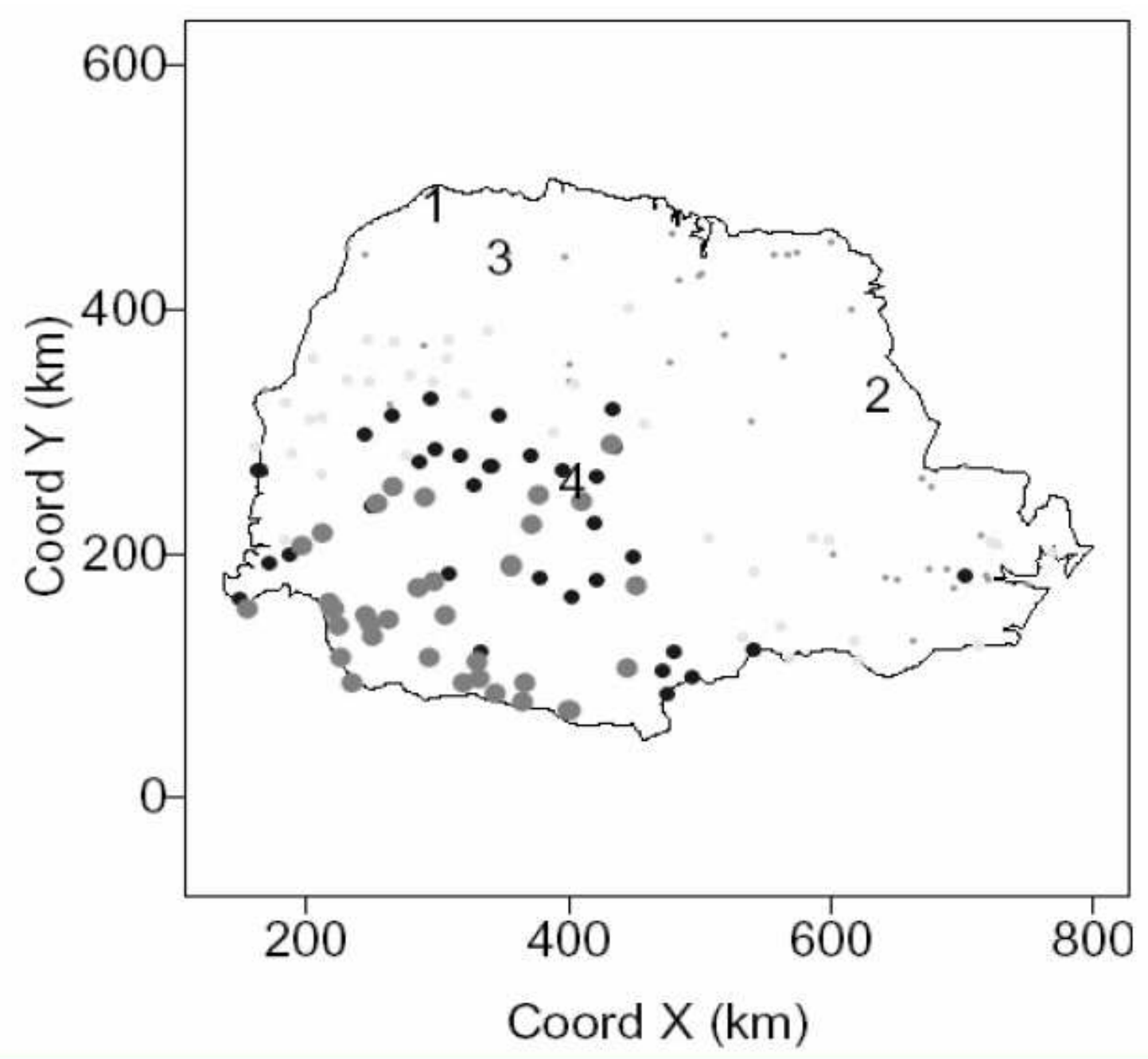




\section{Krigeage des pluies Parana}

Modèle : régression affine et résidu Gauss + Pépite

- à gauche carte des hauteurs de pluie (données en •)

- à droite carte des écarts types des prédictions
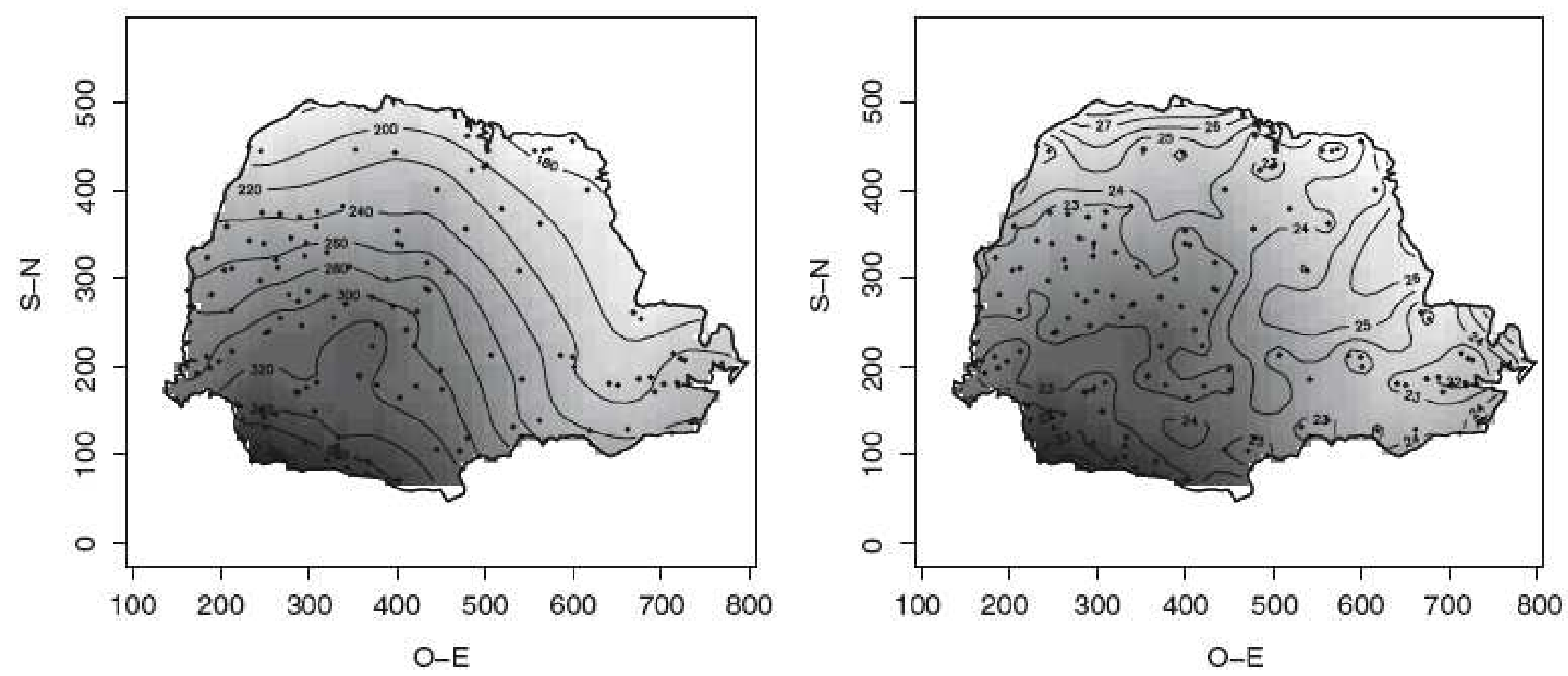

Atelier Spatial RASMA

Saint Louis du Sénégal 


\section{Propriété de la surface de Krigeage}

1. Interpolateur universel : krigeage $\equiv$ observation en un point d'observation

2. Régularité de la surface de krigeage fonction de la régularité du variogramme (covariance) en 0 :

- Pépitique : krigeage constant partout $\equiv$ la moyenne arithmétique des observations, discontinuité aux points d'observation.

- Linéaire pointu en 0 : surface continue mais non dérivable aux points d'observations.

- Parabolique en 0 : continue et dérivable partout. 


\section{Régularité du Krigeage et régularité à l'origine de la covariance spatiale}

Exemple d'école : 2 observations en ( $x$ ) et 3 reconstructions
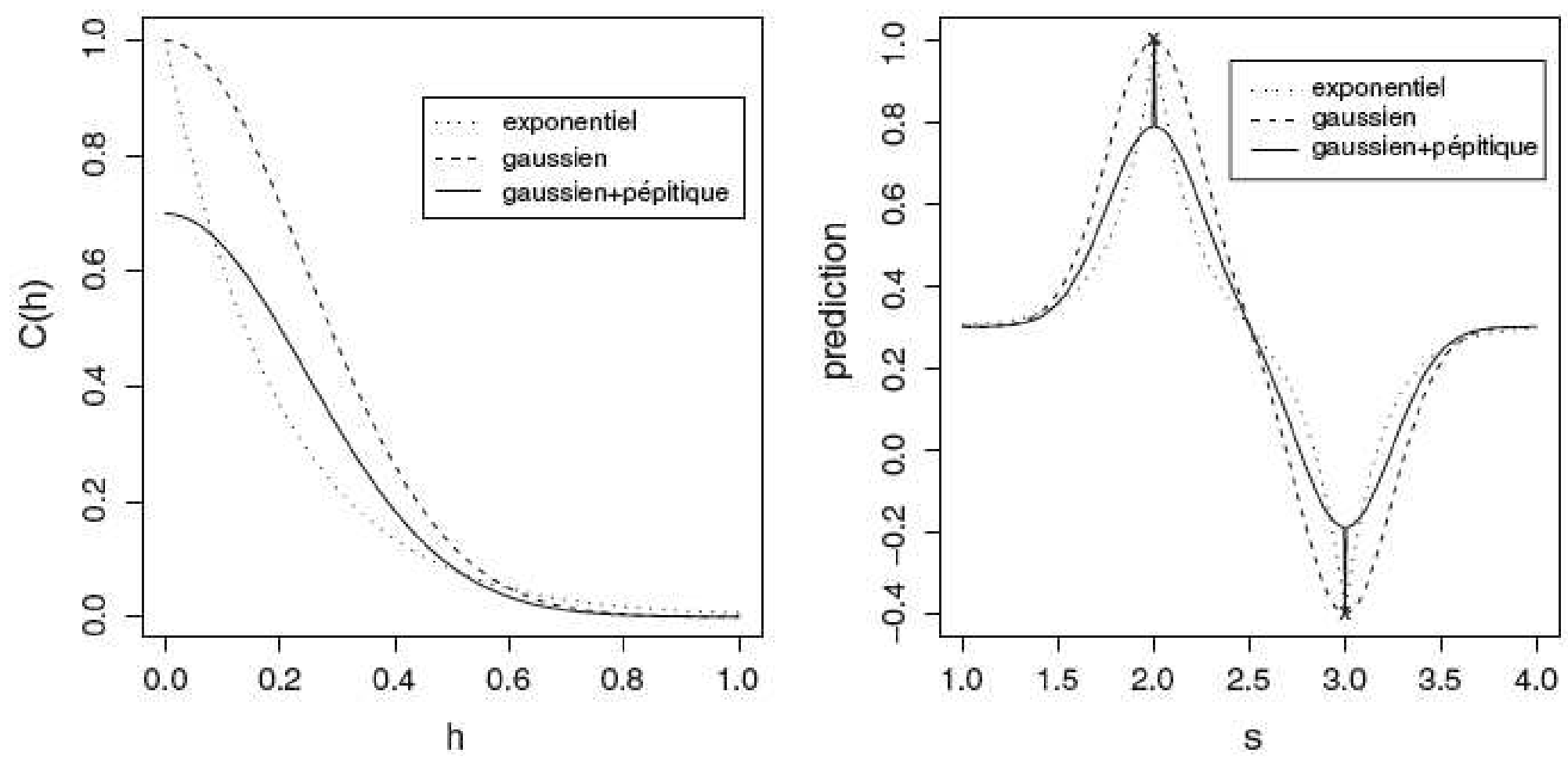
Prédictions sur un intervalle à partir de 10 points

Deux variogrammes : exponentiel (continu) et Matérn (pointillé);

Deux dispositifs : en haut, points régulièrement espacés; bas, placés au hasard
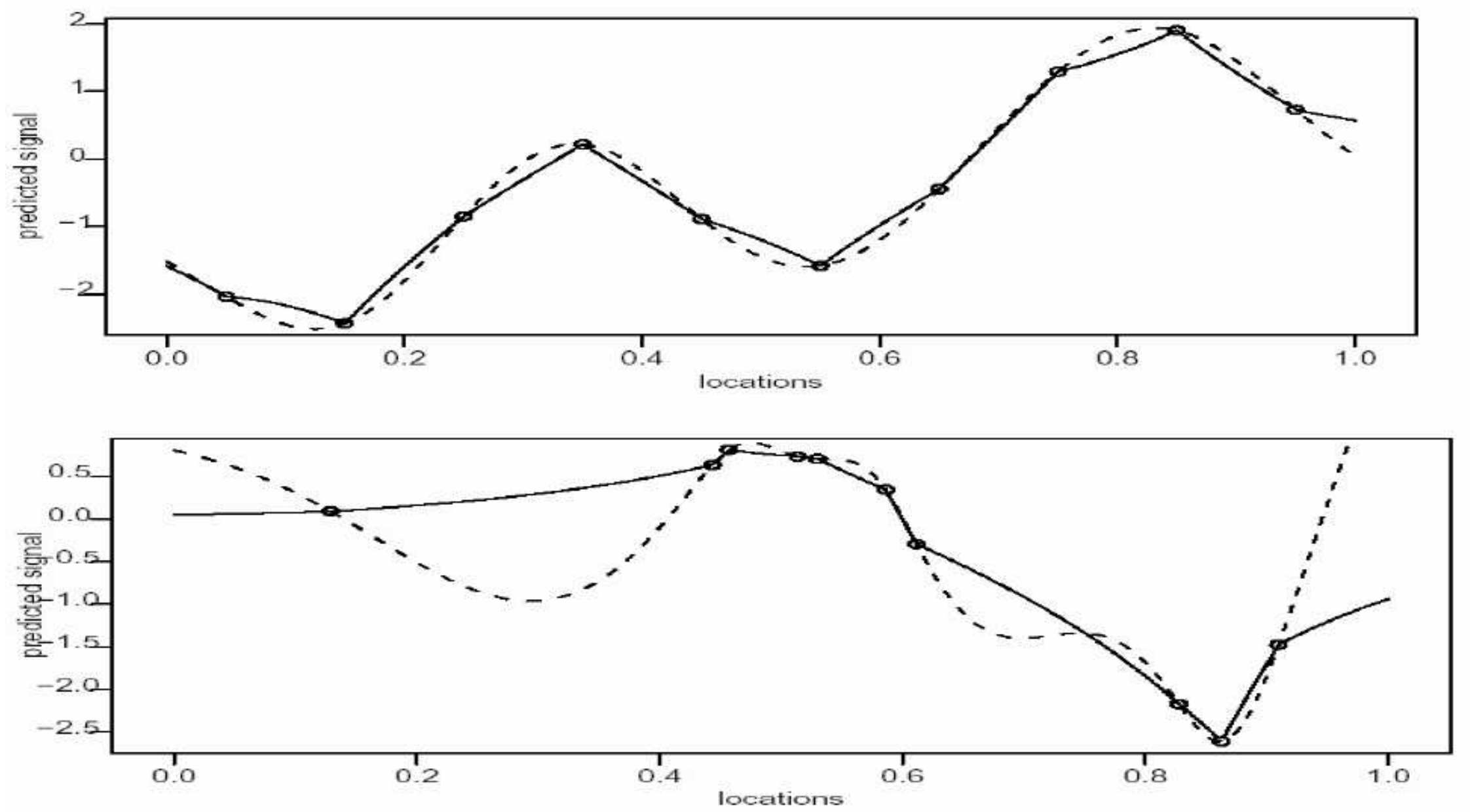


\section{Krigeage ou simulation conditionnelle}

(a) X simulé, gaussien, cov. exponentielle

et

(b) 25 points échantillonnés

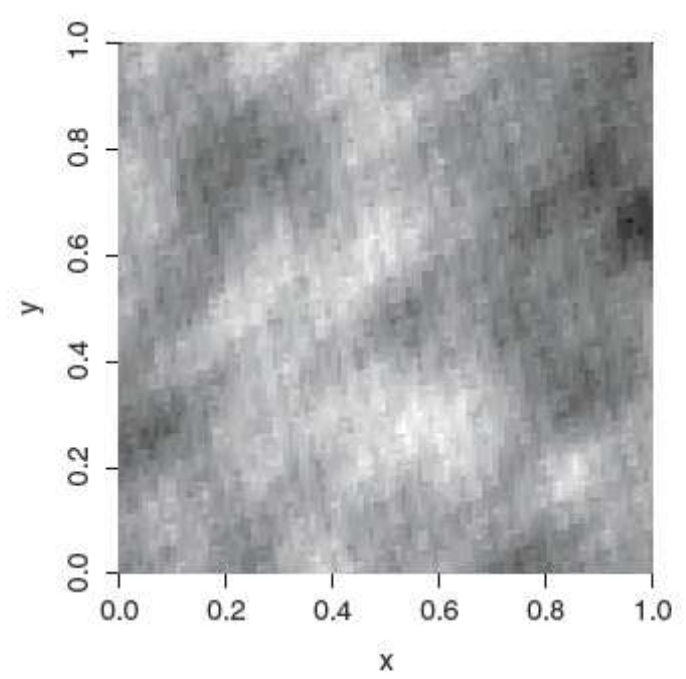

(a)

(c) reconstruction de $X$ par

krigeage

(d) reconstruction de X par simulation conditionnelle aux 25 observations

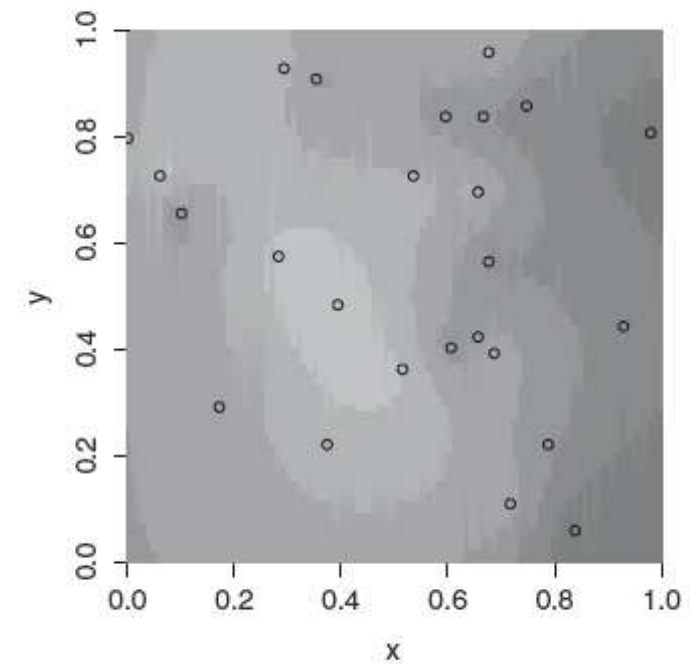

(c)

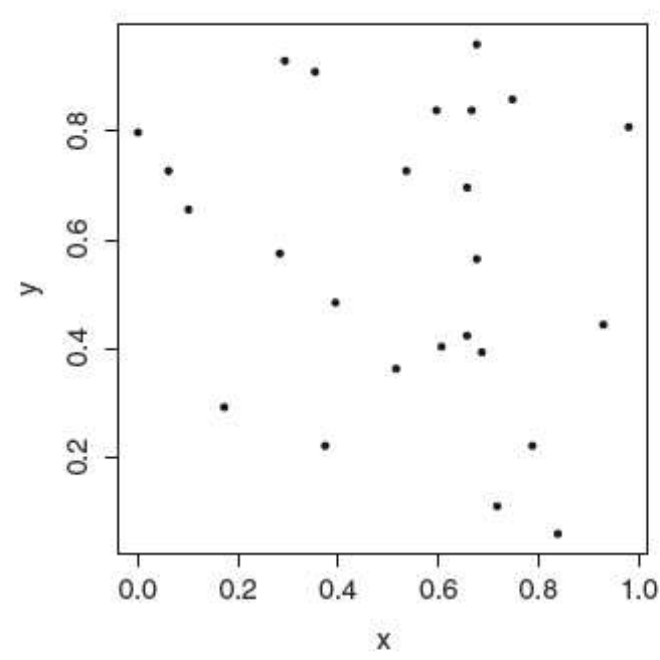

(b)

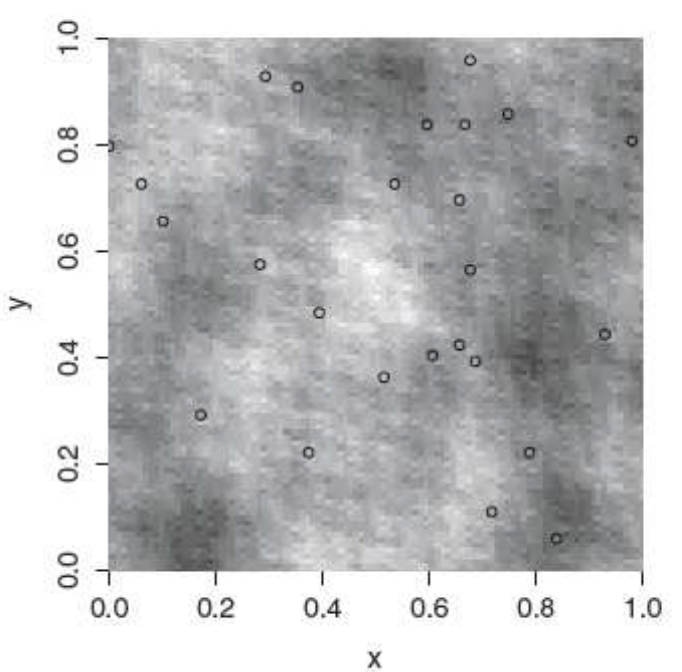

(d) 


\section{Simulation conditionnelle à 100 points}

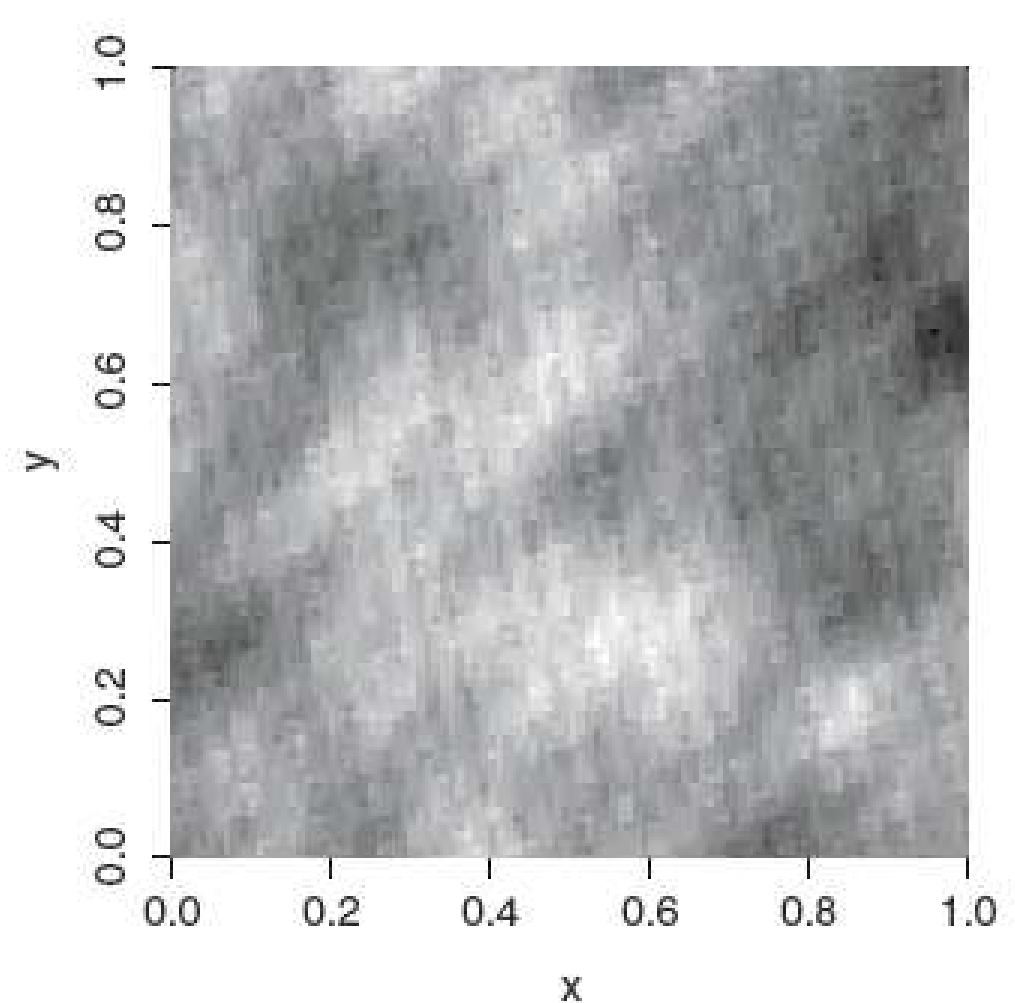

$X$ initial

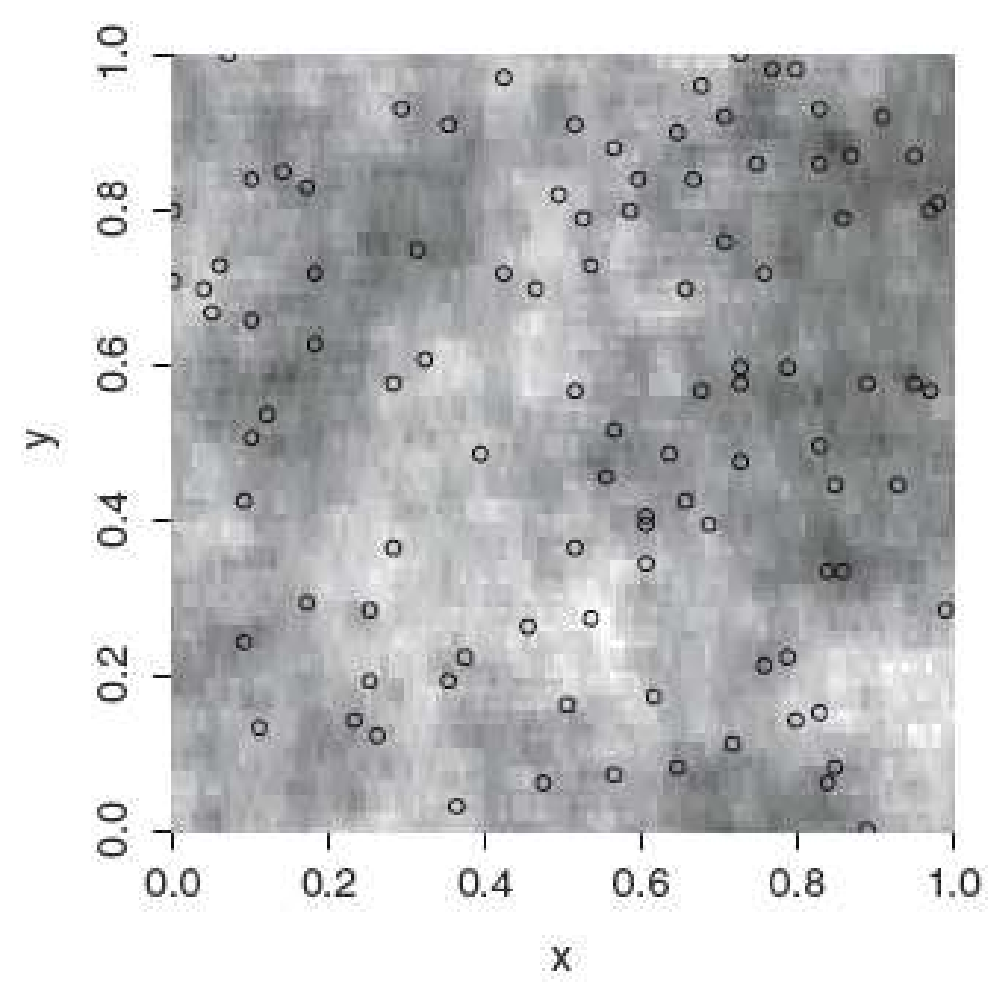

simulation cond. 


\section{Statistique des modèles de géostat}

- Deux niveaux : au premier ordre (moyenne, tendance) et au deuxième ordre (covariance, variogramme).

- Statistique sans modèle : estimations empiriques.

- Statistique avec modèle : MCO, MCP, MCG ou MV.

- Validation et choix de modèle : validation croisée, Bootstrap paramétrique ou méthode de Monte Carlo. 


\section{Nuée variographique (cas isotropique)}

$$
\begin{aligned}
& \text { Observation de } X \text { en } n \text { points } \mathcal{O}=\left\{s_{1}, s_{2}, \cdots, s_{n}\right\} \\
& \text { Nuée variographique }=\text { nuage des } \frac{n(n-1)}{2} \text { points : } \\
& \qquad \mathcal{N}=\left\{\left(\left\|s_{i}-s_{j}\right\|,\left(X_{s_{i}}-X_{s_{j}}\right)^{2}, i \neq j\right\}\right. \\
& \text { Comme } E\left(\left(X_{s_{i}}-X_{s_{j}}\right)^{2}\right)=\gamma\left(s_{i}-s_{j}\right), \\
& \Longrightarrow \mathcal{N} \text { "estime" sans biais } h \mapsto \gamma(h)
\end{aligned}
$$

- Plus lissage avec bon noyau de convolution

- si $X$ non isotropique, nuées dans les 4 directions cardinales $\{E, N E, N, N W\}$ avec une tolérance angulaire de $\pm 22.5^{\circ}$ 


\section{Estimation empirique du variogramme}

1 - $N(h)$ : ensembles des couples $r$-voisins à $\Delta$-près (au moins 30 points dans chaque $N(h)$ )

2 - Avantage : ne nécessite pas d'estimation préalable de la moyenne (nécessaire pour estimer une covariance)

3 - Version robuste aux grandes valeurs $(X(s(i))-X(s(j))) * * 2$ (Cressie et Hawkins)

$$
\begin{gathered}
\widehat{\gamma}_{n}(h)=\frac{1}{\# N(h)} \sum_{\left(s_{i}, s_{j}\right) \in N(h)}\left(X_{s_{i}}-X_{s_{j}}\right)^{2}, \quad h \in \mathbb{R}^{d} . \\
N(h)=\left\{\left(s_{i}, s_{j}\right): r-\Delta \leq\left\|s_{i}-s_{j}\right\| \leq r+\Delta ; i, j=1, \ldots, n\right\} .
\end{gathered}
$$




\section{Estimation empirique du variogramme : Choix de classes de distances et effectifs par classes}
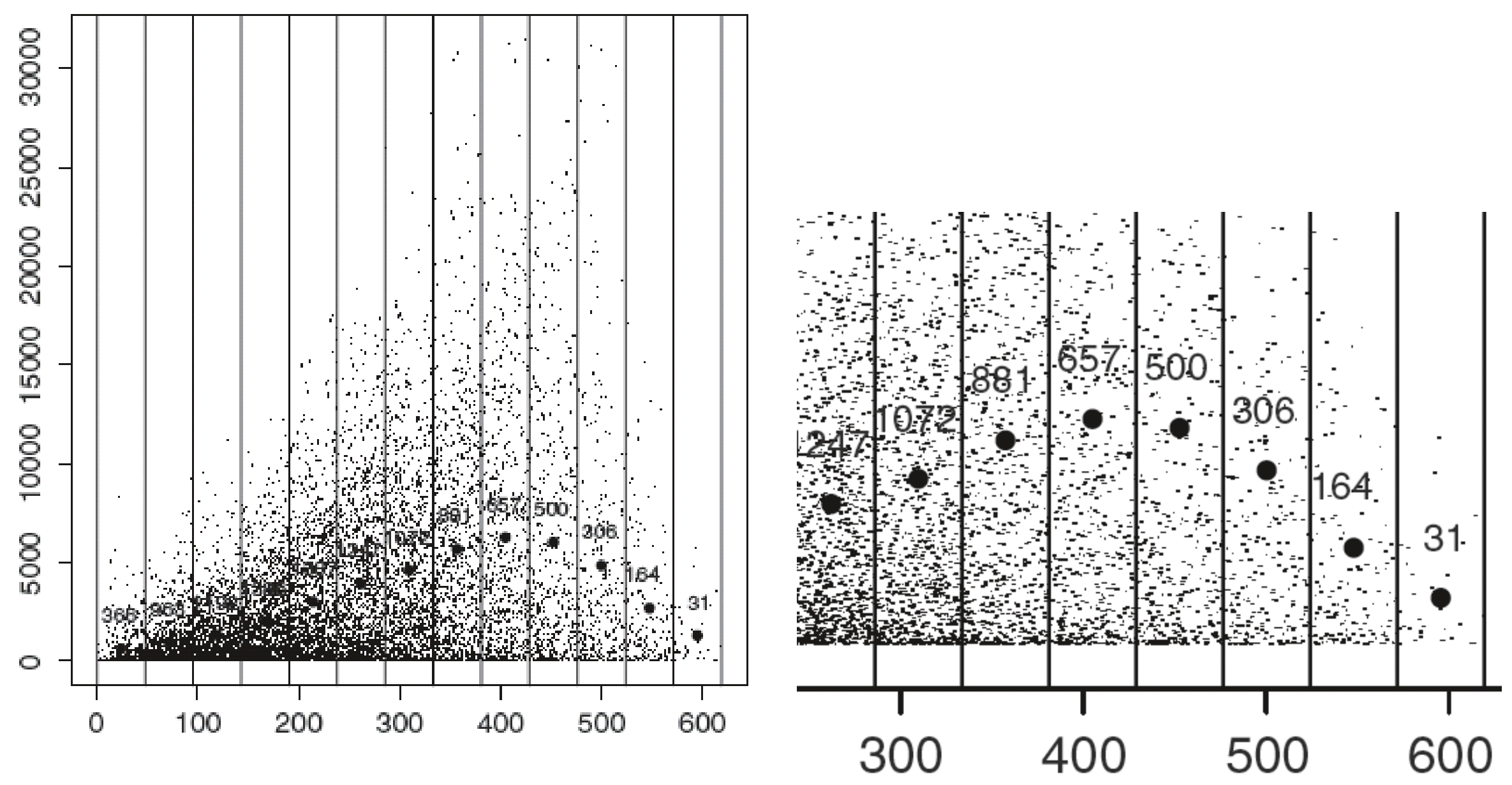

Atelier Spatial RASMA

Saint Louis du Sénégal 


\section{Nuée variographique et variogrammes lissés}

\section{(données de pluies au Parana)}

(a) modèle isotropique

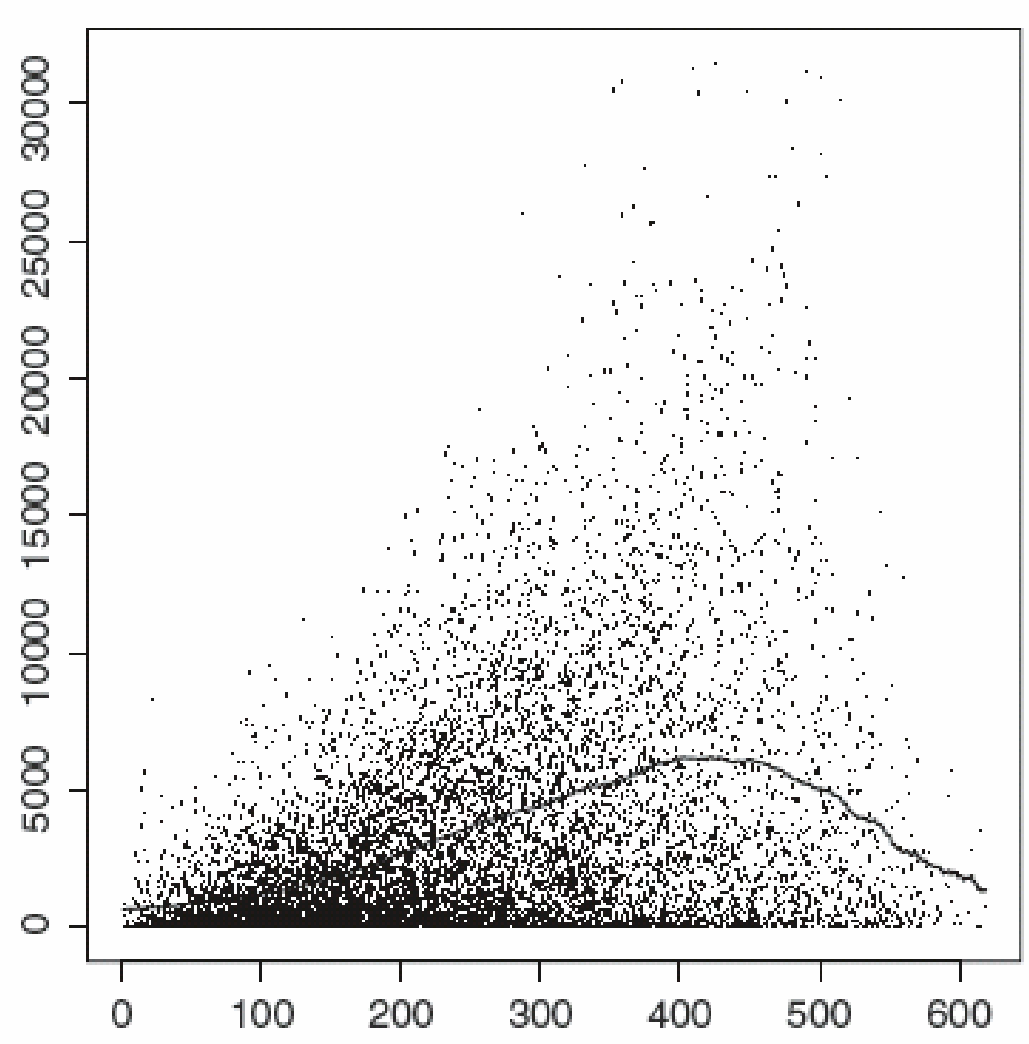

(a) (b) dans 4 directions

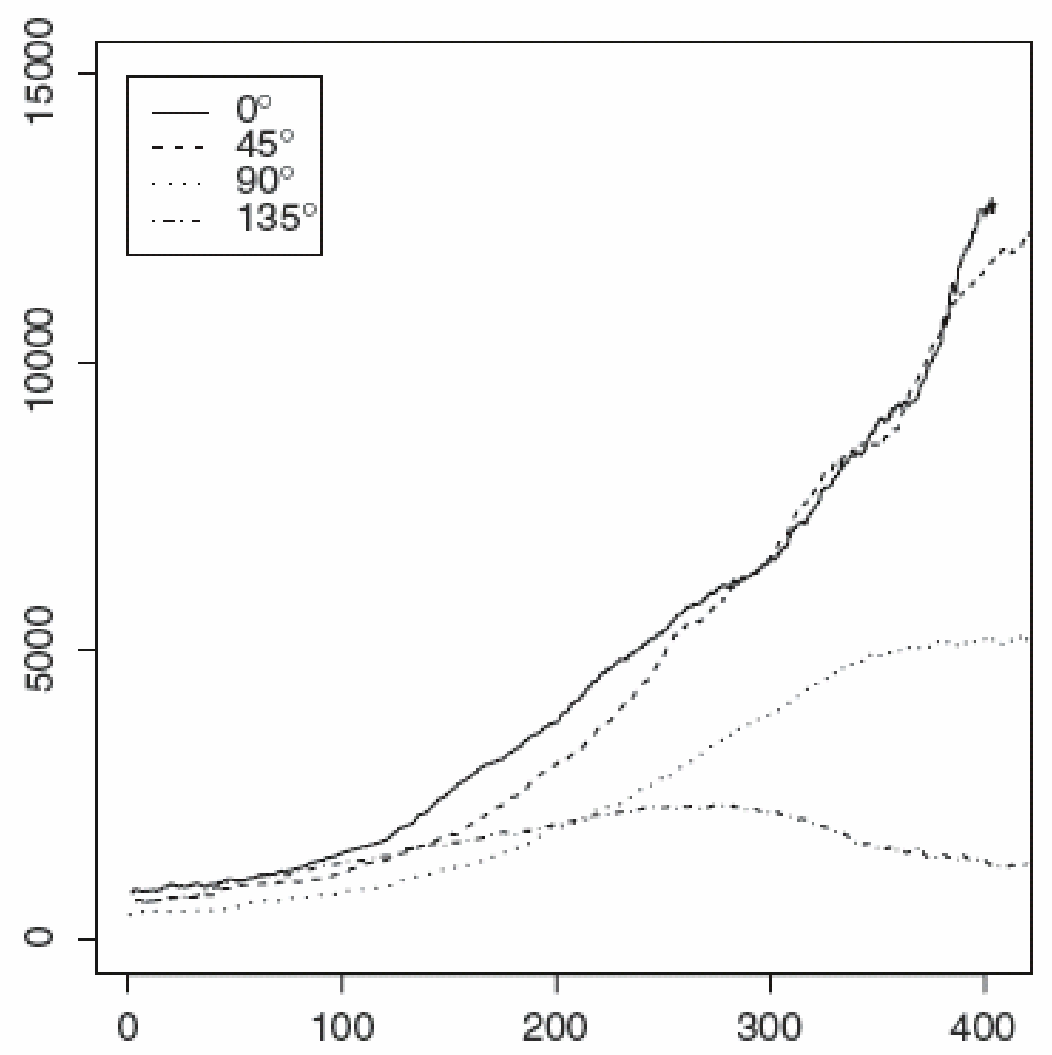

(b) 


\section{variog : calcul du variogramme empirique}

- isotrope ou non (variog4),

- Fixation ou non du nombre de classes (bin)

- estimation classique ou robuste

Retourne une estimation par classe (bin), le nuage variographique, le variogramme lissé ...

$>\operatorname{variog}($ ca20)

$>$ print $(x)$

$>\operatorname{plot}(\mathrm{x})$ 


\section{Données Parana : ajustement affine}

Rouges : au dessus

Noirs : au dessous

$\rightarrow$ calcul des résidus puis variogramme des résidus

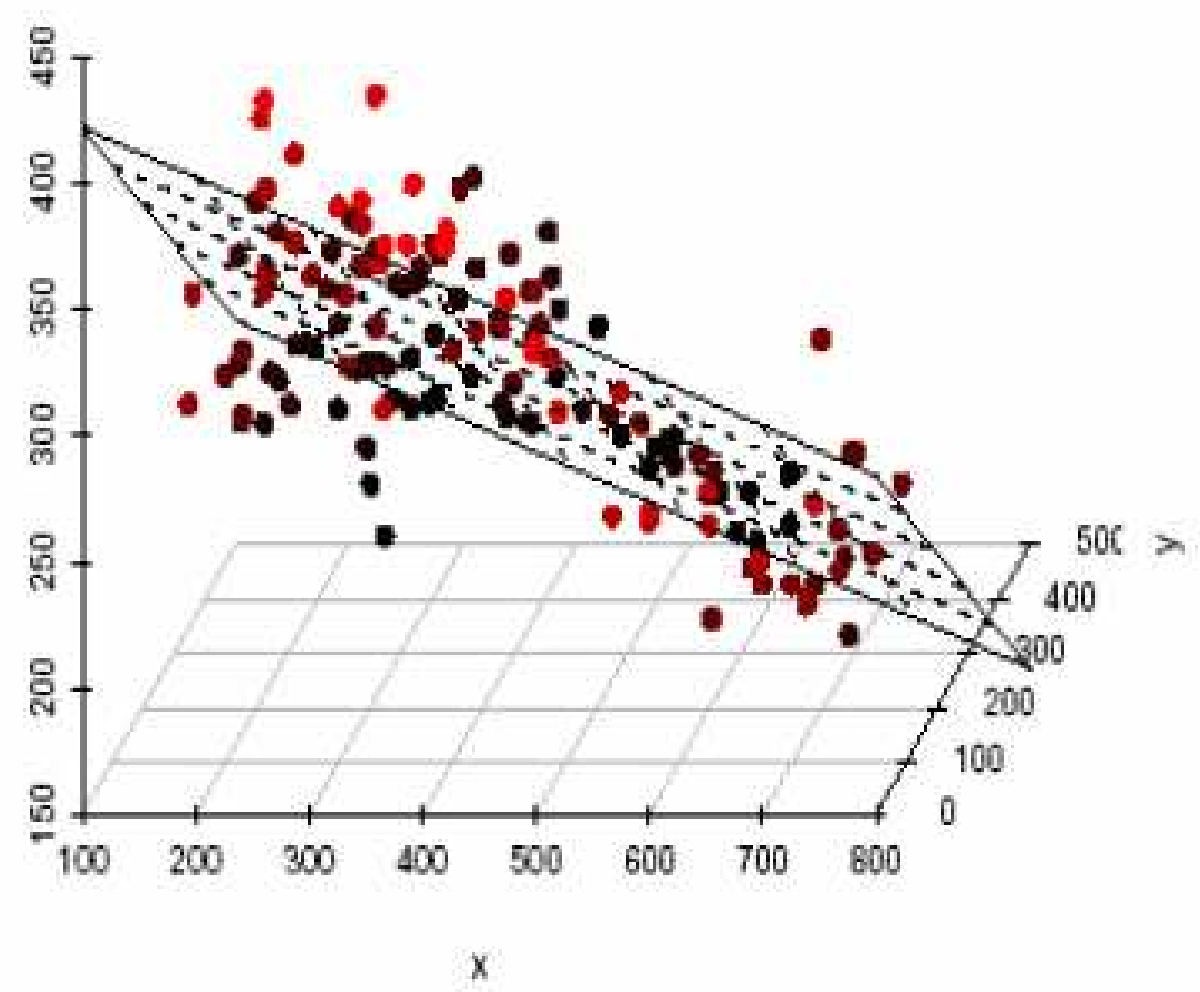




\section{Variogrammes des résidus dans $\mathbf{4}$ directions pour:}

(c) le modèle moyen affine (pluies au Parana);

(d) le modèle moyen quadratique
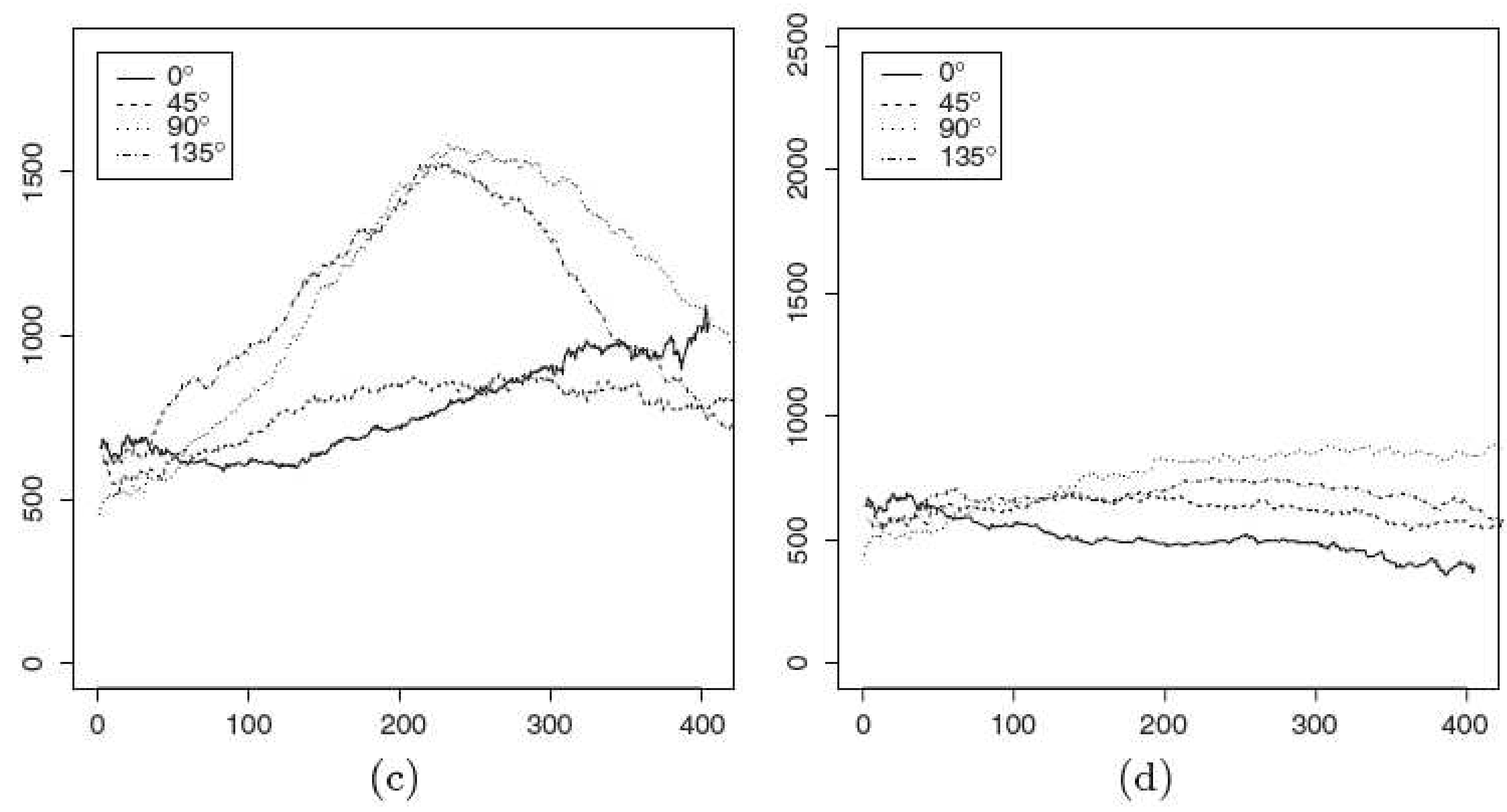

Atelier Spatial RASMA

Saint Louis du Sénégal 


\section{Estimation d'un modèle paramétrique :}

Choisir $\mathbf{k}$ classes de distances rendant identifiable le modèle de variogramme :

$$
\text { variogramme } \gamma(\cdot ; \theta)
$$

$$
k \text { classes } \mathcal{H}=\left\{h_{1}, h_{2}, \cdots, h_{k}\right\}
$$




\section{Estimations d'un modèle de variogramme}

1 - Moindres carrés ordinaires (MCO)

2 - Moindres carrés pondérés (MCP)

3 - Moindres carrés généralisés (MCG) et MCQG

4 - Maximum de Vraisemblance (MV)

$$
\begin{gathered}
\widehat{\theta}_{M C O}=\underset{\alpha \in \Theta}{\operatorname{argmin}} \sum_{i=1}^{k}\left(\widehat{\gamma}_{n}\left(h_{i}\right)-\gamma\left(h_{i} ; \alpha\right)\right)^{2} \\
\widehat{\theta}_{M C P}=\underset{\alpha \in \Theta}{\operatorname{argmin}} \sum_{i=1}^{k} \frac{\# N\left(h_{i}\right)}{\gamma^{2}\left(h_{i} ; \alpha\right)}\left(\widehat{\gamma}_{n}\left(h_{i}\right)-\gamma\left(h_{i} ; \alpha\right)\right)^{2} \\
\widehat{\theta}_{M C G}=\underset{\alpha \in \Theta}{\operatorname{argmin}}{ }^{t}\left(\widehat{\gamma}_{n}-\gamma(\alpha)\right)\left\{\operatorname{Cov}_{\alpha}\left(\widehat{\gamma}_{n}\right)\right\}^{-1}\left(\widehat{\gamma}_{n}-\gamma(\alpha)\right)
\end{gathered}
$$




\section{Estimation du variogramme en présence d'une tendance linéaire}

$$
X_{s}={ }^{t} z_{s} \delta+\varepsilon_{s}, \quad \delta \in \mathbb{R}^{p}
$$

résidu champ intrinsèque centré,

$$
E\left(\varepsilon_{s+h}-\varepsilon_{s}\right)^{2}=2 \gamma(h, \theta), \quad \theta \in \mathbb{R}^{q} .
$$

1. Estimer par MCO

2. En déduire les résidus des $M C O$

3. Variogramme de ces résidus $M C O$ 


\section{Estimations modèles de pluies en Suisse}

A gauche : données (stations et intensité);

A droite : estimations empiriques classique et robuste sur 13 classe de distances.
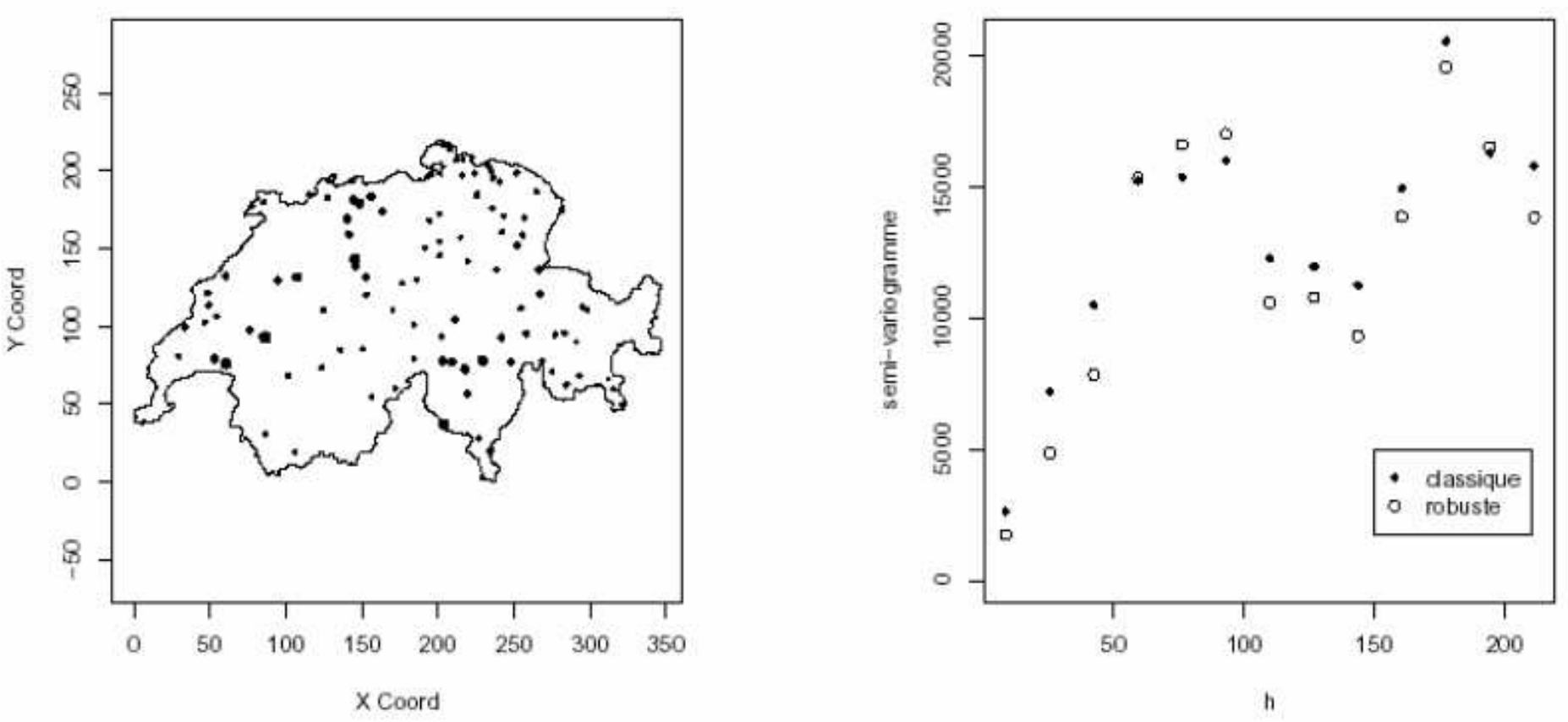


\section{Estimation du modèle de Matern}

(a) $\mathrm{MCO}, \mathrm{MCP}$ et $\mathrm{MV}$ du variogramme de Matern

(b) Estimations empiriques + enveloppes sup et inf à partir de $\mathrm{m}=40$ simulations du modèle de Matern estimé par MCP

(intervalle de confiance à $1-2 /(m+1)=95 \%$ )

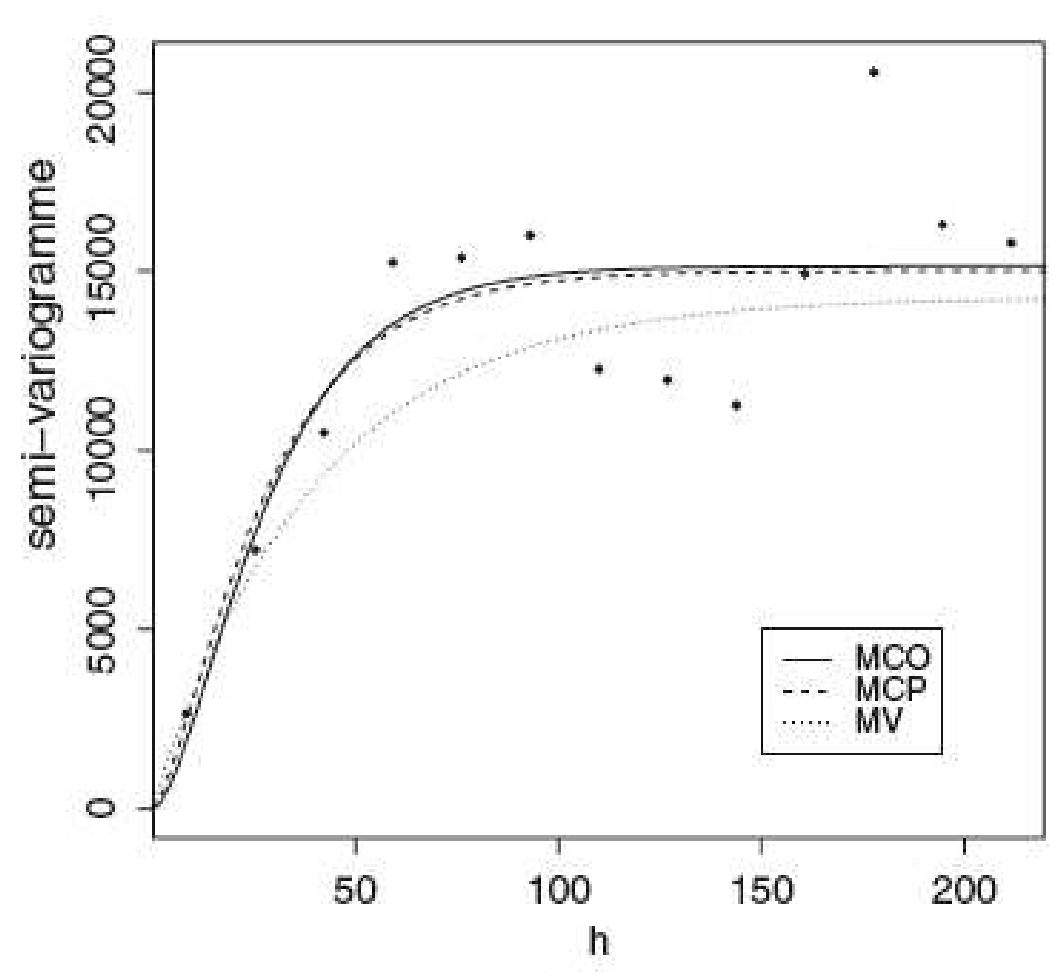

(a)

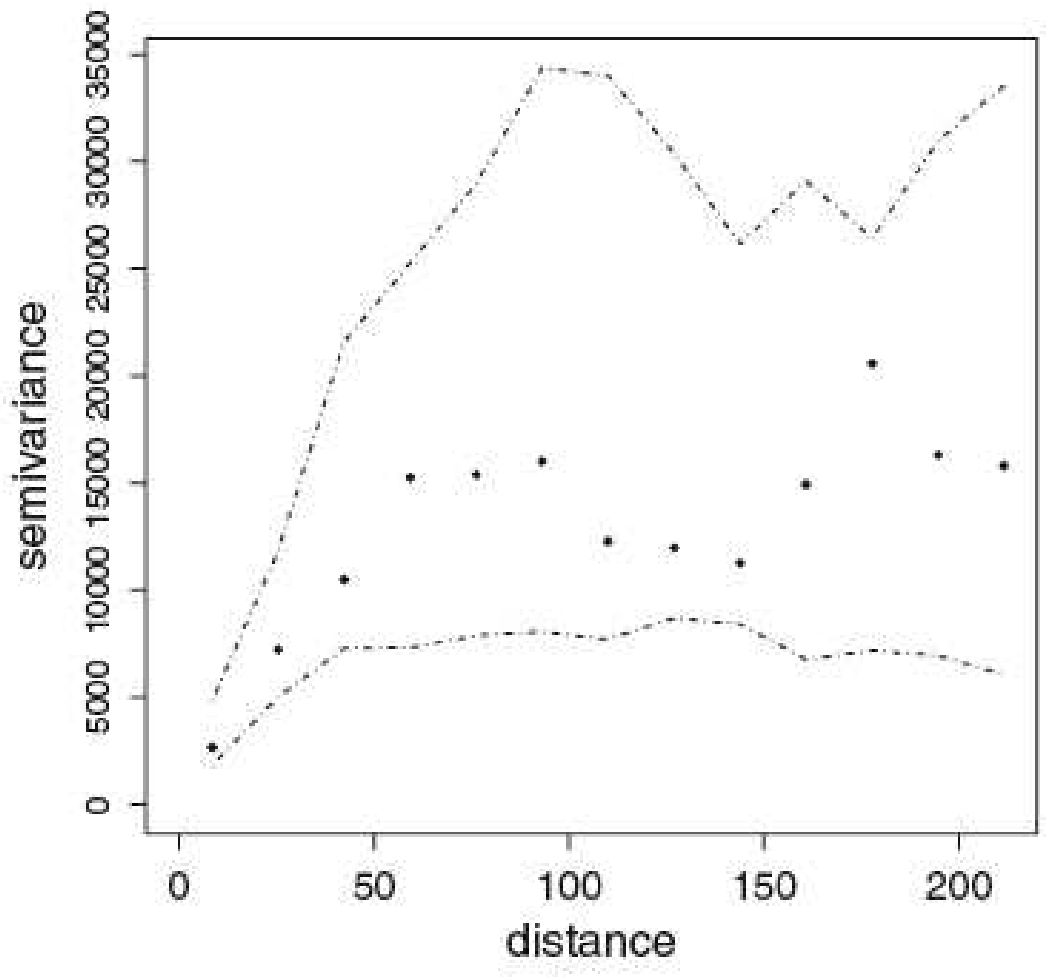

(b) 


\section{variofit et likfit: estimation d'un variogramme paramétrique}

- variofit

estime les paramètres d'un modèle de covariance (variogramme) par MCO ou par MCP, ceci à partir du variogramme empirique (variog)

- likfit

réalise l'estimation par maximum de vraisemblance

- Exercice : sur un jeu de données (personnelles), estimer un modèle paramétrique par MCO et par MV. Représentations simultanées des estimations empiriques et paramétriques. 


\section{Krigeage pour le modèle de Matern}

\section{(données suisses)}
(a) carte des pluies et
(b) de leurs écarts types

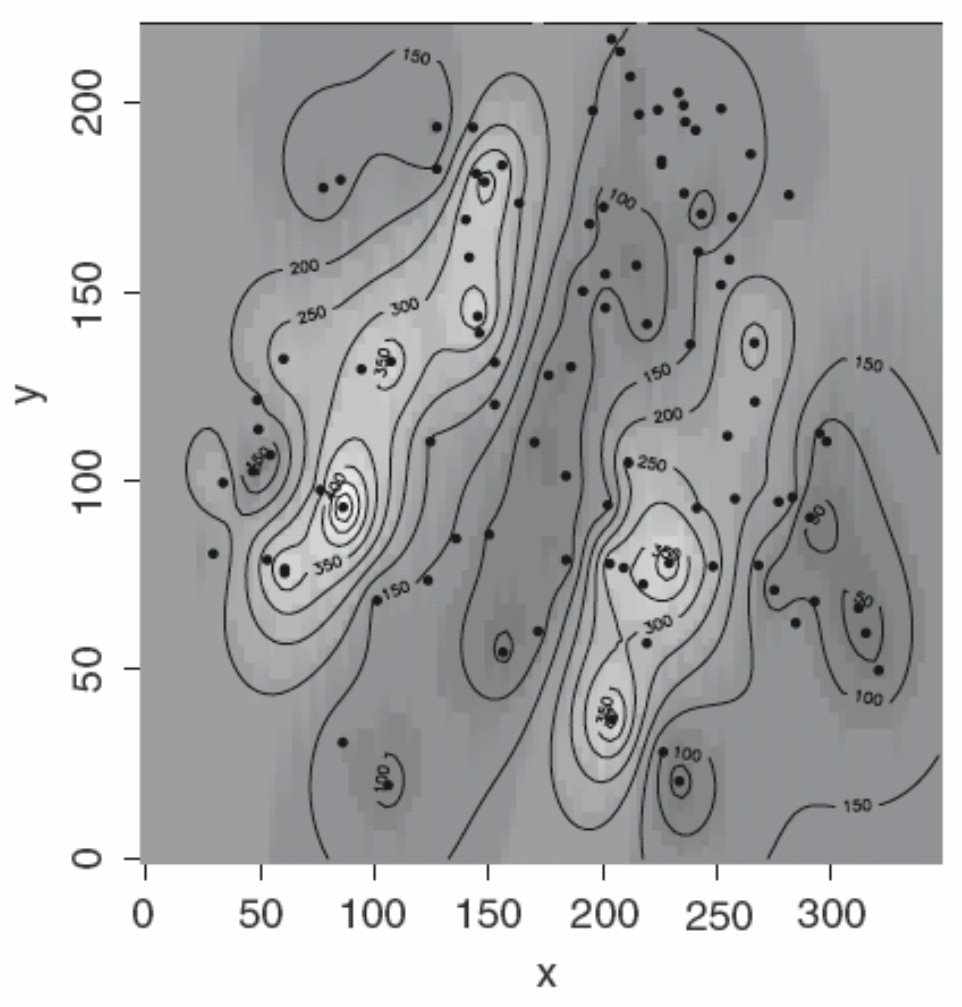

(a)
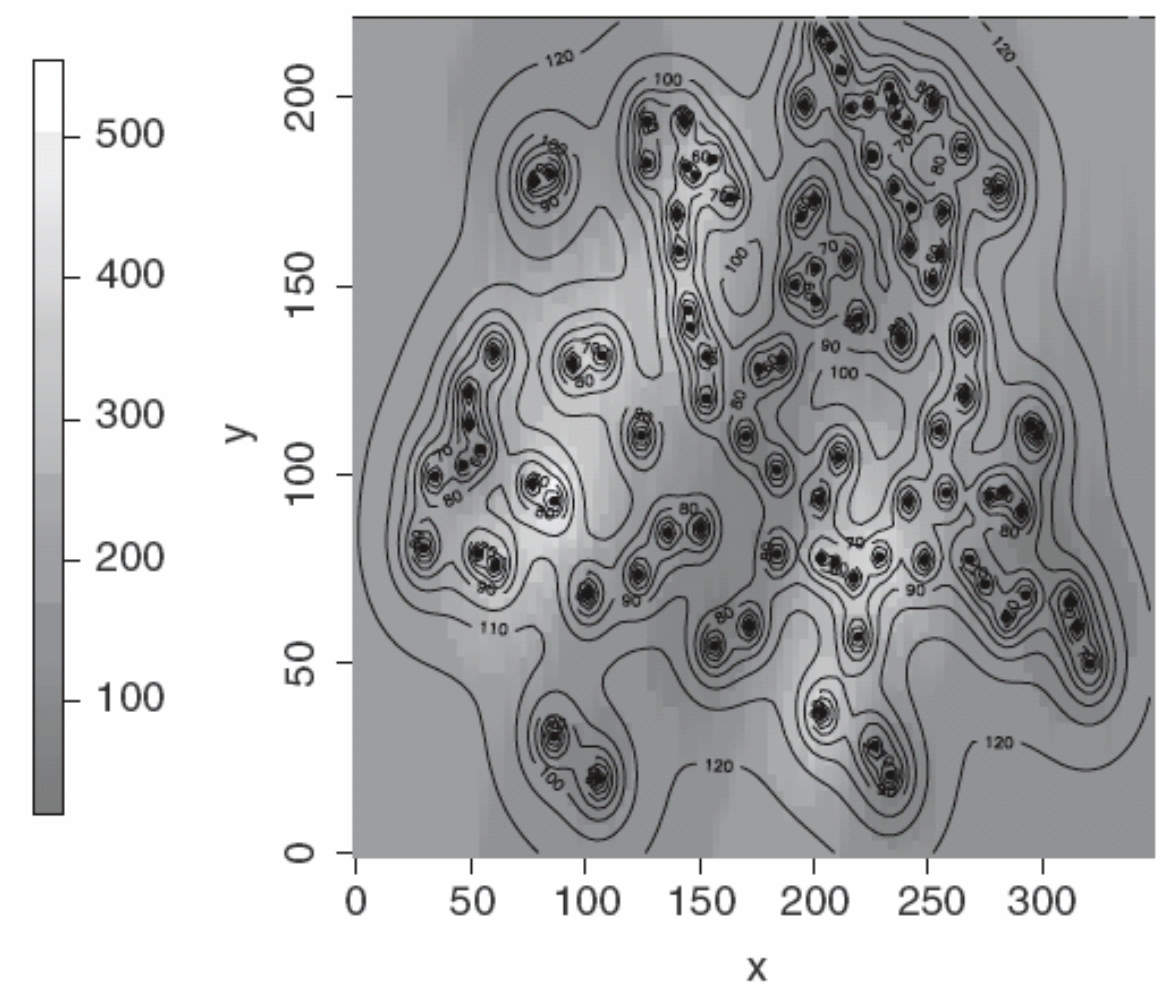

(b) 


\section{Exemple : densité de pêche de la coquille Saint Jacques dans l'atlantique nord}
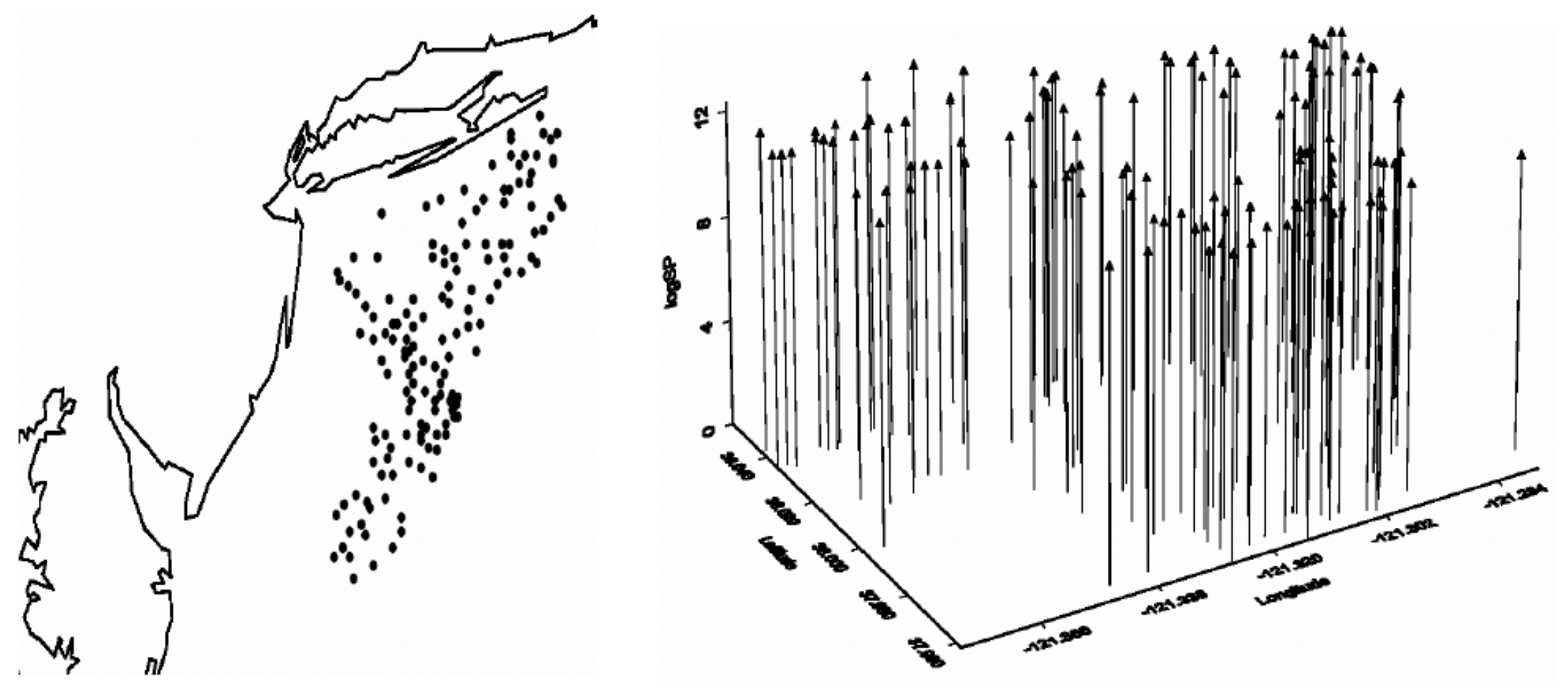


\section{Données de "Pêche de coquilles saint Jacques » Variogrammes directionnels et rose d'anisotropie.}
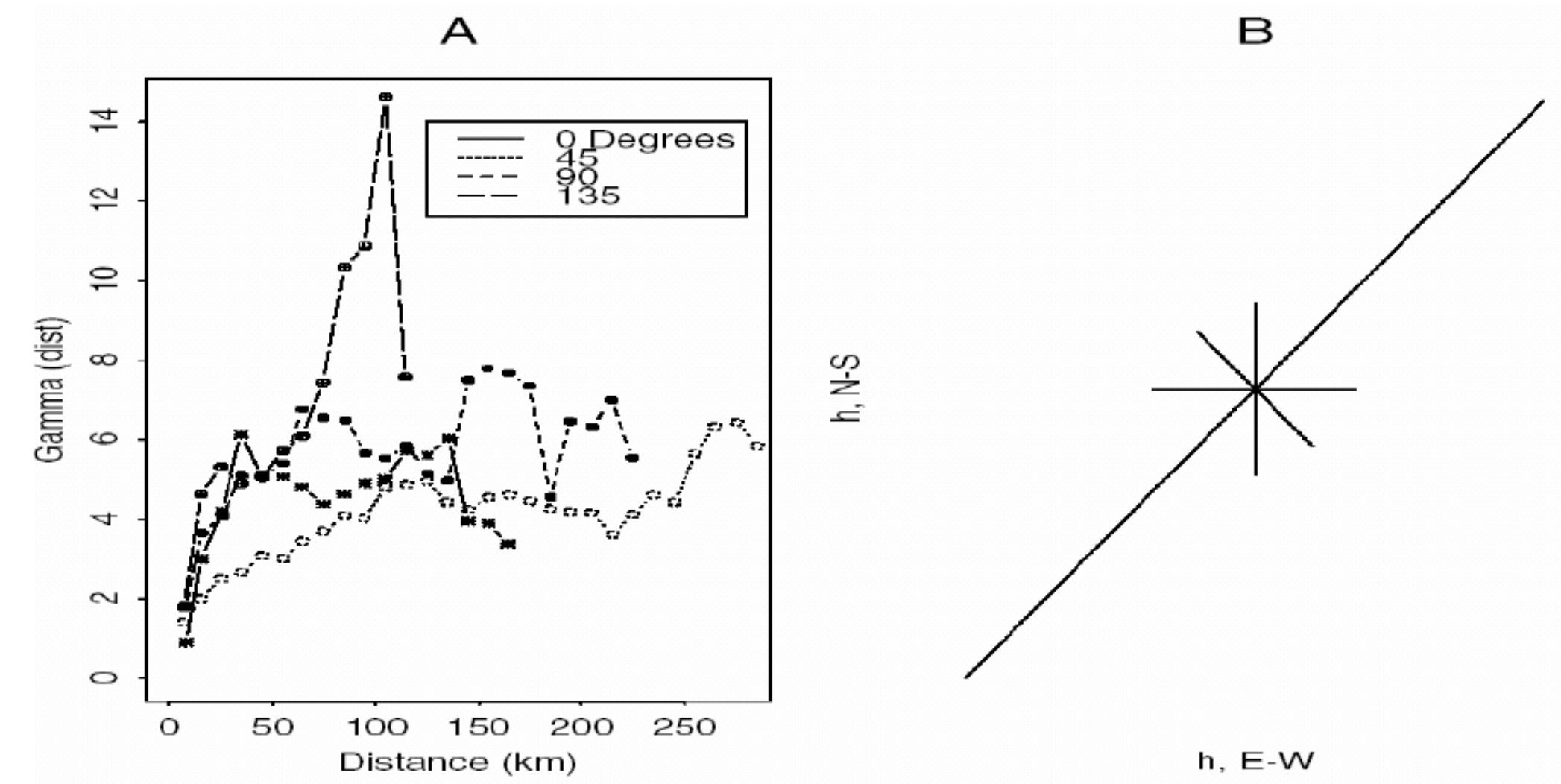


\section{Estimation du modèle « sphérique + pépitique 》}

( après transformation isotropiante )

Estimation empiriques robustes sur 20 classes de distances;

Estimation du modèle « sphérique + pépitique » : portée $=0.8$; var $=4.8$ et bruit additif de variance 0.7 .

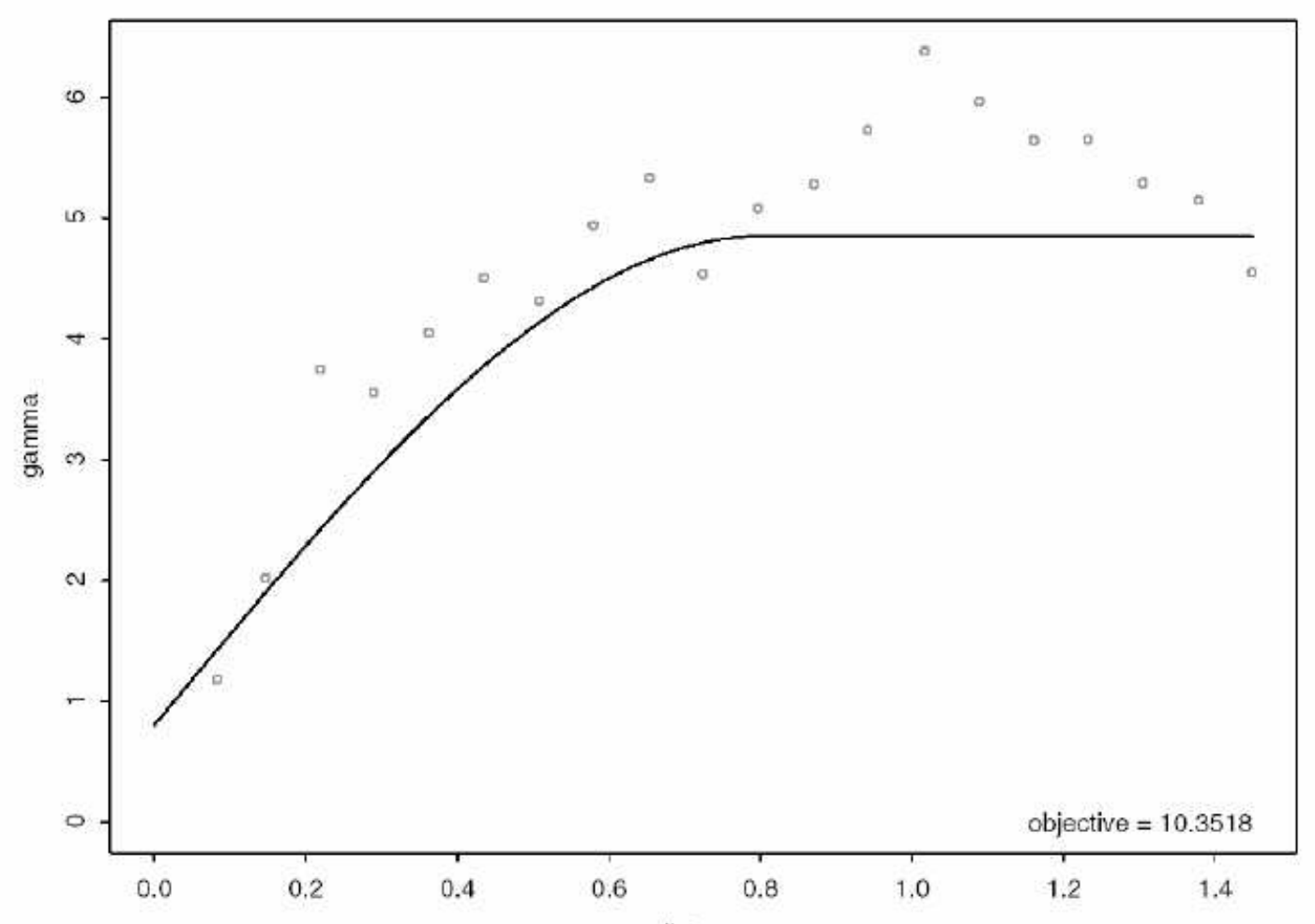

Atelier Spatial RASMA

Saint Louis du Sénégal 
Krigeage : carte de prédiction de la densité en coquille Saint Jacques pour le modèle sphérique estimé.

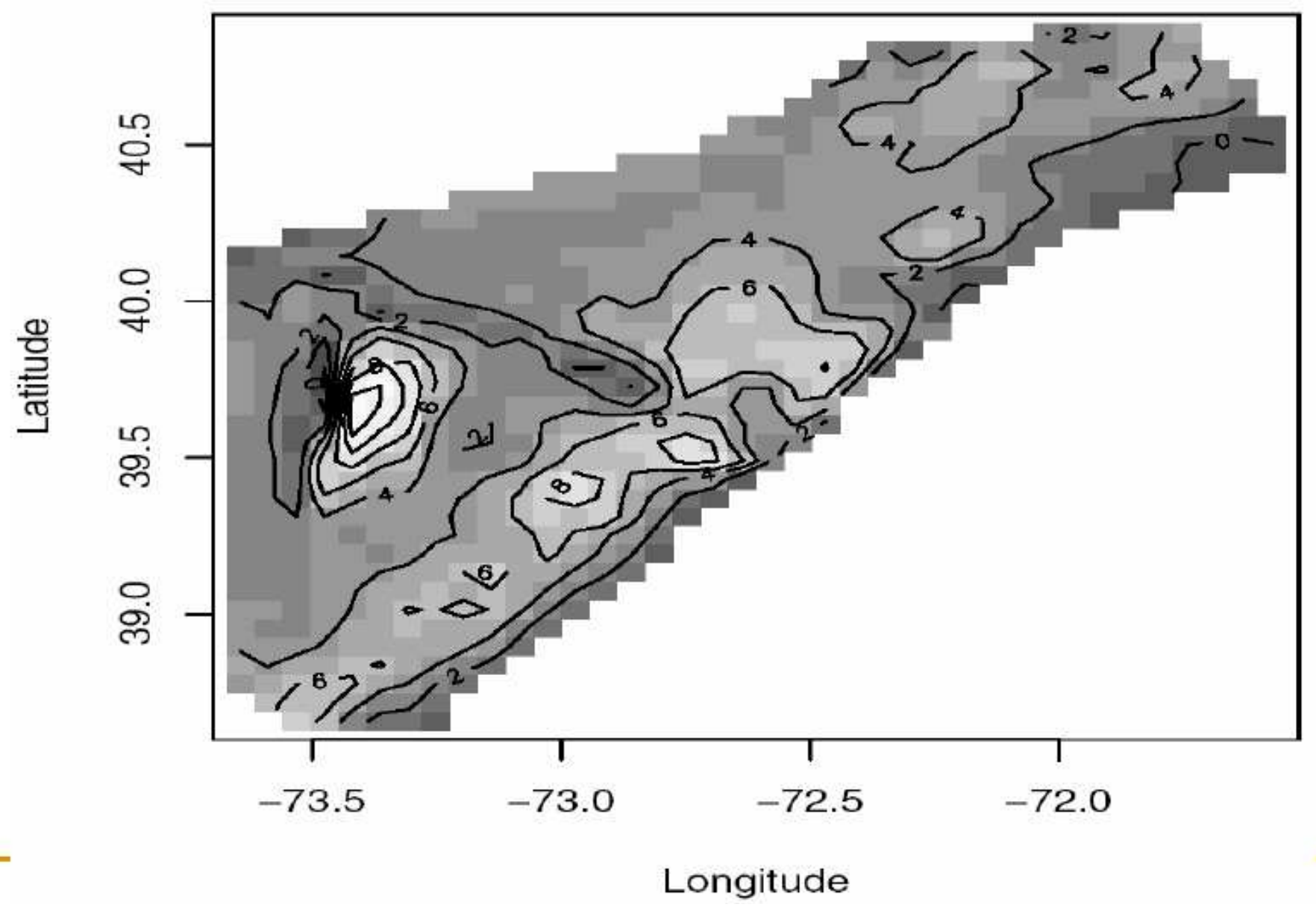

Atelier Spatial RASMA

Saint Louis du Sénégal 


\section{Convergence et normalité asymptotique des estimateurs}

- Choix de $k$ classes de distances identifiant le modèle paramétrique

- Régularité suffisante de la fonctionnelle d'estimation

- Faible dépendance (mélange) du champ géostatistique

- Extension des résultat au cas où il existe une tendance paramétrique (paramètres d'ordre 1, moyenne) et 2 (variogramme des résidus).

$\rightarrow$ Voir les résultats (Lahiri, Cressie, C. Gaetan - X. G.) 


\section{Validation d'un modèle paramétrique}

- Validation croisée

- Validation par Bootstrap paramétrique 


\section{Validation croisée d'un variogramme}

- Estimer le modèle paramétrique

- Éliminer à tour de rôle une observation $x$ (i) et la prédire par krigeage avec le modèle estimé

- Calculer l'Ecart Quadratique Normalisé (par la variance de prédiction) Moyen sur tous les $x(i)$ :

$$
E Q N M=\frac{1}{n} \sum_{i=1}^{n} \frac{\left(X_{s_{i}}-\widehat{X}_{s_{i}}\right)^{2}}{\tilde{\sigma}_{s_{i}}^{2}}
$$

- Si I'EQM proche de 1, valider le modèle (à 95\% de sécurité) :

$$
|E Q N M-1| \leq 1.96 \sqrt{\frac{2}{n}} \text {. }
$$




\section{Exemple : validation du modèle de Matern}

(données suisses)

Modèle de Matérn et trois méthodes d'estimation (MCO,

MCG et MV) pour données « pluies suisses ».

\begin{tabular}{|c|cccc|}
\hline & $\hat{a}$ & $\hat{\sigma}^{2}$ & $\hat{\nu}$ & EQNM \\
\hline MCO & 17.20 & 15135.53 & 1.21 & 1.37 \\
MCP & 18.19 & 15000.57 & 1.00 & 1.01 \\
MV & 13.40 & 13664.45 & 1.31 & 1.09 \\
\hline
\end{tabular}




\section{Validation par bootstrap paramétrique}

- Modèle paramétrique $\rightarrow$ estimation du paramètre

- m simulations des données sous le modèle estimé

- pour chaque simulation $\rightarrow$ variogramme empirique

- enveloppes inférieure + supérieure des variogrammes empiriques

$\rightarrow$ bande de confiance au niveau $\alpha=(1-2 /(m+1))$

- Si le variogramme empirique des données initiales dans la bande de confiance, le modèle est validé. 


\section{Validation du modèle de Matern}

\section{(données Parana)}

- $m=40$ simulations sous le modèle de Matern estimé

- intervalle de confiance à 95\% pour le variogramme aux 13 distances

- contient les variogrammes empiriques $\rightarrow$ modèle de Matern valide

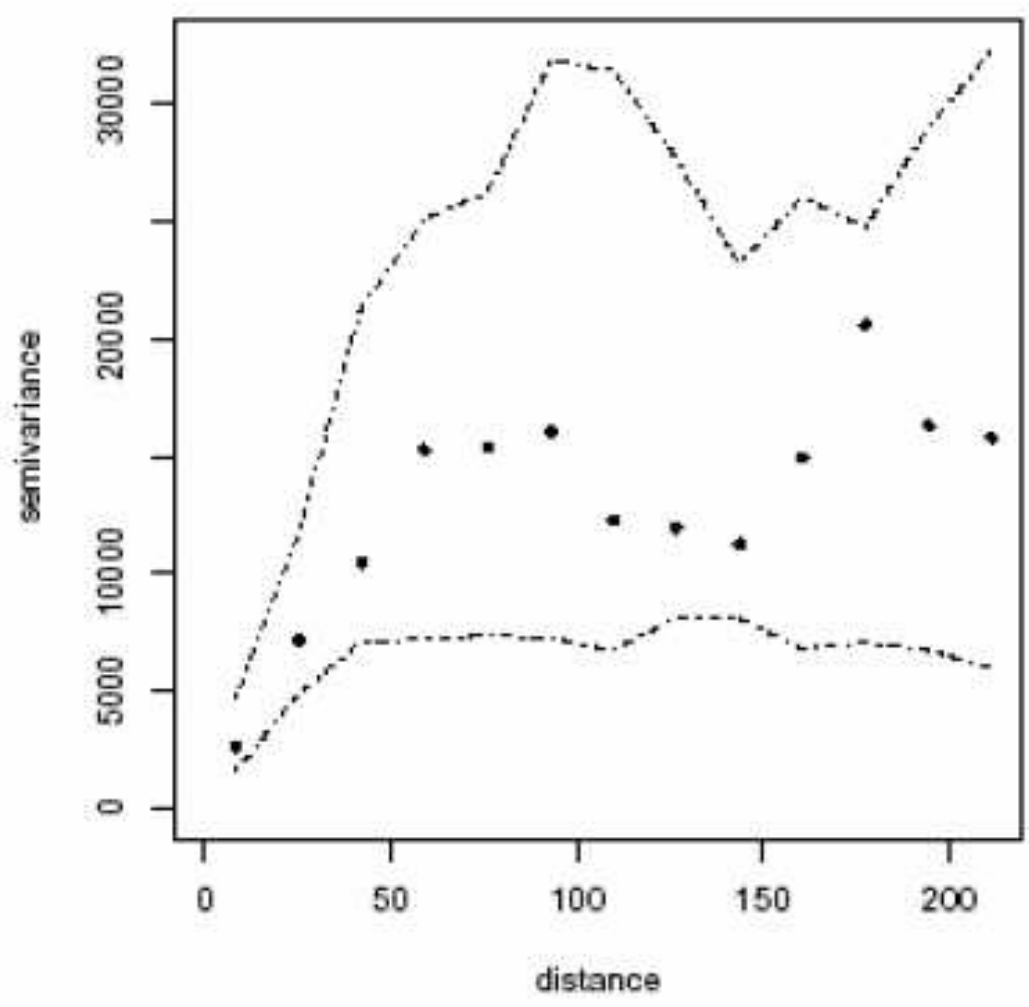

Atelier Spatial RASMA

Saint Louis du Sénégal 


\section{Bande de confiance, validation de modèle}

- variog.model.env

Répétition de simulations d'un modèle (donné ou estimé) aux sites d'observations (modèle gaussien)

$\rightarrow$ variogrammes empiriques pour chaque simulation

$\rightarrow$ bande de confiance pour le variogramme

$\rightarrow$ si variogramme théorique dans la bande $\rightarrow$ modèle valide

- xvalid

validation croisée via le krigeage : chaque observation est comparée à sa prédiction à partir des autres) 


\section{Une étude épidémiologique : prévalence du paludisme chez l'enfant \\ (donnée gambi a de geoR, Diggle et altri)}

- Expliquer la prévalence du palud (\% d'enfants malades) dans un village $s$ Covariables :

$\rightarrow$ Existence ou non d'un centre de santé primaire

$\rightarrow$ Un indice de végétation (données satellitaires)

$\rightarrow$ Moustiquaire ou non

$\rightarrow$ Si oui, traitée ou non

- Question : existe-t-il un facteur de risque spatial ? 


\section{Données malaria en Gambie}

1 - localisations $s$ des villages échantillonnés

2 - prévalence prop $=y / n$ ( $y=n b$ de malades, $n$ nb d'enfants)

3 - nuace (areen.nron) nour indice de vécétation areen

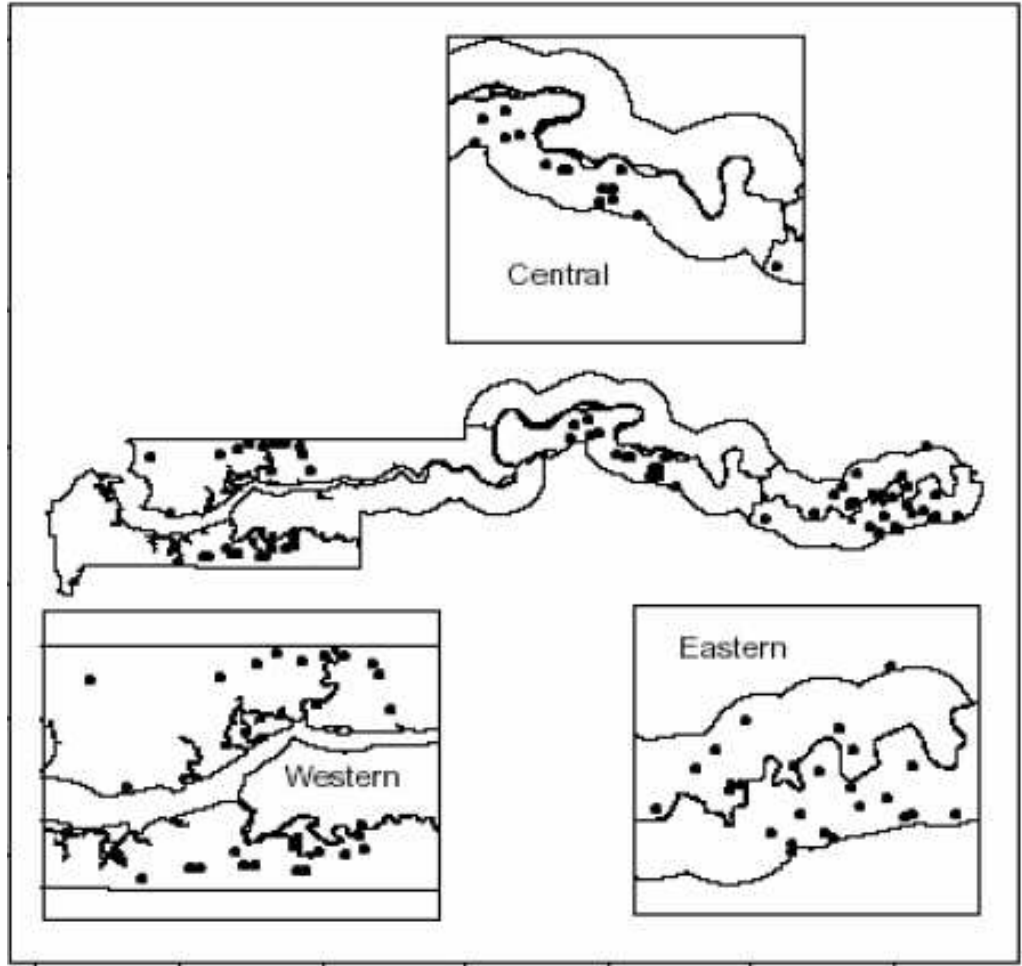

10000350000400000450000500000550000600000
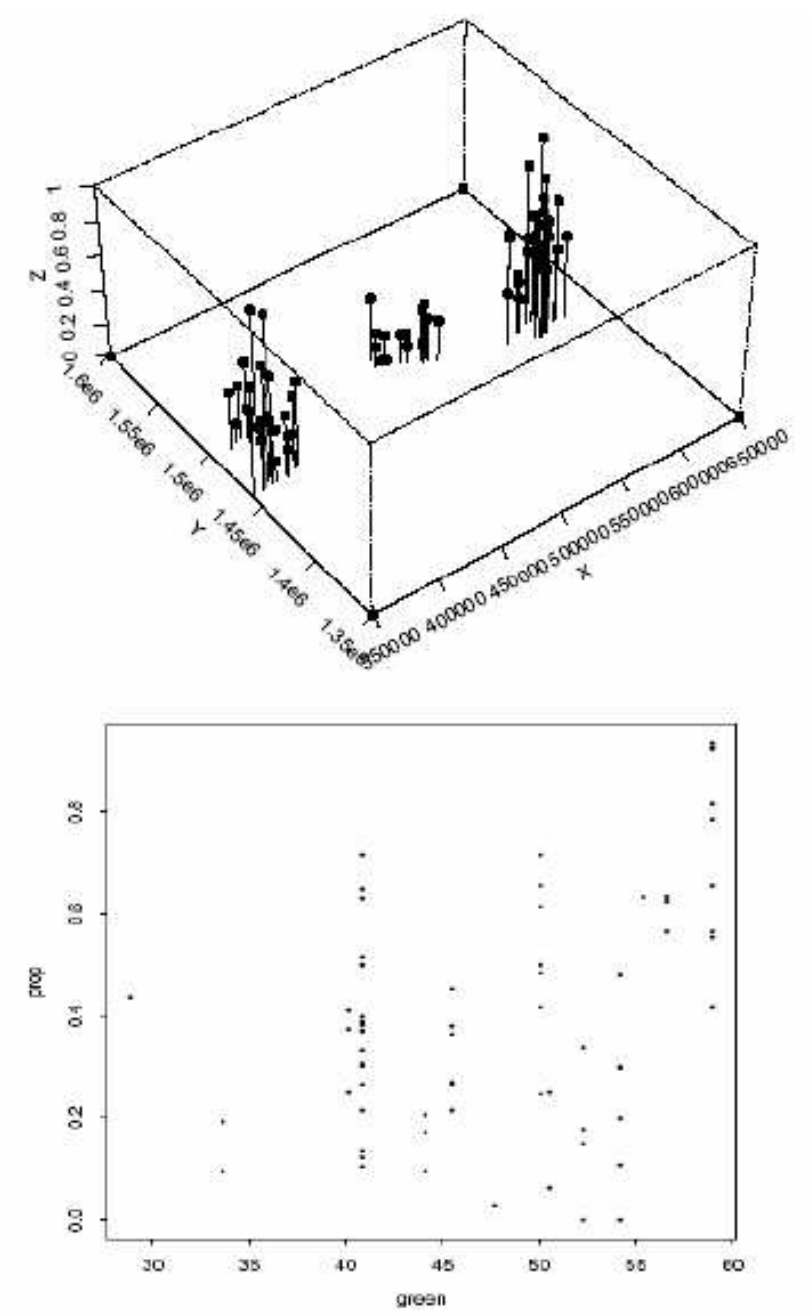

Atelier Spatial RASMA 


\section{Le modèle log-linéaire d'étude}

$\circ Y_{i, s}=$ présence / absence du parasite dans le sang enfant $i$, village $s$

- $p$ covariables $\left(z_{i, s, k} ; k=1, p\right)$ : age, sexe, moustiquaire, traitée ou non, indice végétation ....

Modèle d'étude proposé : régression Logistique

$$
\text { Logit } P\left(Y_{i, s}=1\right)=\sum_{k=1}^{p} z_{i, s, k} \beta_{k}+U_{s}+S(x(s))
$$

où $U_{s} \sim N\left(0, \nu^{2}\right)$ un résidu BBG habituel

$\{S(x): x \in$ Gambie $\}$ est un champ gaussien stationnaire

Analyse :

- présence de $S(x)$ affecte fortement l'estimation de $\beta$

- la carte de $\widehat{S}(x)$ montre un facteur de risque spatial

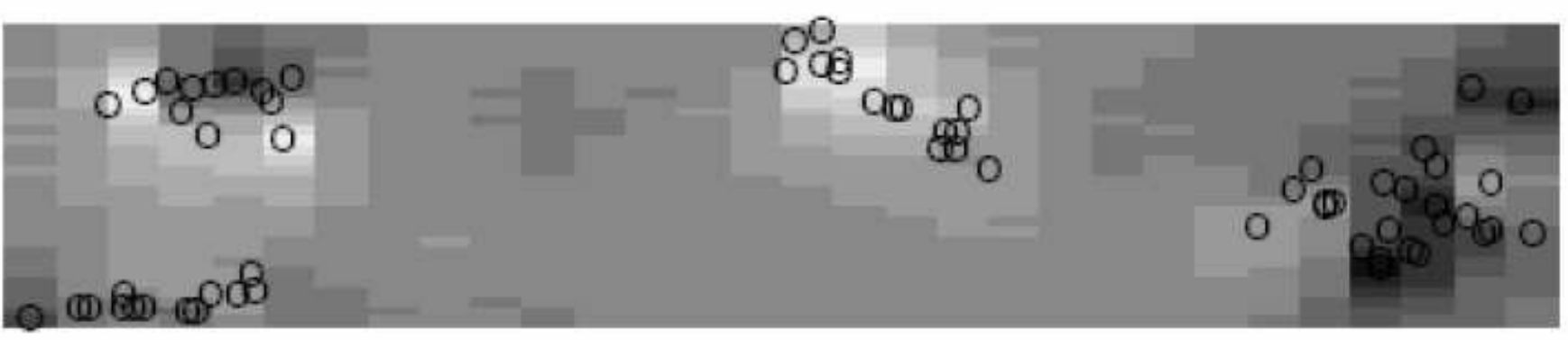

Saint Louis du Sénégal 


\section{Géostatistique : bilan}

- Inventaires des variables influentes disponibles

- Analyse graphiques préalables pour réduire leur nombre

- Propositions de modèles à l'ordre 1 (la tendance) et 2 (la covariance ou le variogramme).

- En particulier, choix a priori de régularité du variogramme
- Nuée(s) variographiques: isotropie ou non?

- Estimation empirique puis paramétrique

- Validation de modèle (validation croisée, bootstrap paramétrique)

- Krigeage simple ou universel

- Cartes de krigeage : prédiction et précision.... 


\section{Géostatistique avec $R$ :}

geoR, gstat, geoRlm et RandomFields

\section{geoR}

- Nombreuses données (gambia, parana, etc ...)

- Quelques exemples de programmes :

geoR2RF : simulation d'un GMF (Gaussian Markov Field).

likefit: $\mathrm{MV}$ pour un GRF.

krige.conv: krigeage conventionnel.

plotvariogram : variogramme empirique.

variofit: MV /MC d'un modèle à partir du variogramme empirique.

$\boldsymbol{x v a l i d}$ : validation croisée d'un modèle de variogramme; etc ... 


\section{Géostatistique avec $\boldsymbol{R}$ (suite)}

geoRglm : general linear spatial modèle.

gstat: Modélisation, estimation, prédiction en géostatistique uni-multidimensionnelle.

Random Fields : simulation et analyse des champs aléatoire $(\mathrm{RF})$ gaussiens. Exemple de " package »:

CondSimul : simulation conditionnelle.

fitvario et mleRF : MC et MV pour modèles de RF. 


\section{Modèle sur un réseau discret SAR ou Champ de Markov}

Deux différences entre ces 2 familles :

(a) Spécificité des états $E$

(b) linéarité ou non du modèle

- Pour AR spatial : $E=\boldsymbol{R}$ ou $\boldsymbol{R}^{\star \star} d+$ modèle « linéaire » d'équations Simultanées (SAR gaussien ou non)

- Pour Champ de Markov: E général (i.e. $\{0,1\}$, fini, $N, \boldsymbol{R}$, $\boldsymbol{R}+, \boldsymbol{R}^{* *} d$ etc...) et modèle conditionnel (en général) non linéaire

Pour $E=R$, les $C A R$ sont aussi Markov 


\section{(I) Champ de Markov}




\section{Champ de Markov sur réseau}

- Valables pour un espace d'états général (pas limité à $\mathbf{R}$ ).

- Lattice régulier (imagerie, agronomie) ou non (épidémiologie, , environnement, etc...)

- Modèle définit par ses spécifications conditionnelles locales.

• Outils spécifiques: simulation MCMC, estimation PVC. 


\section{Exemple de réseau : ei re, 3 réseaux réguliers, et $\mathrm{sids}$}

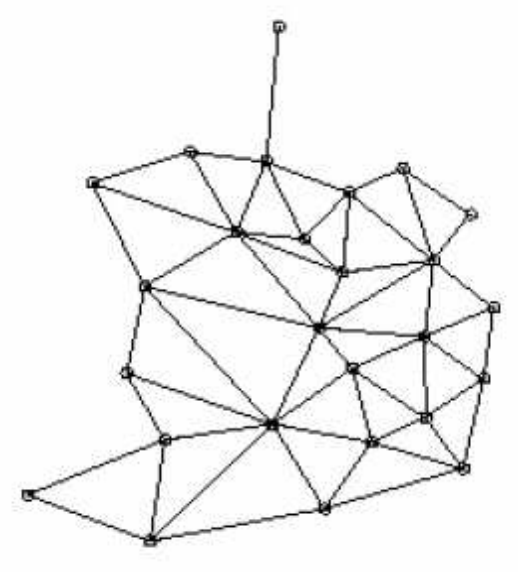

(a)

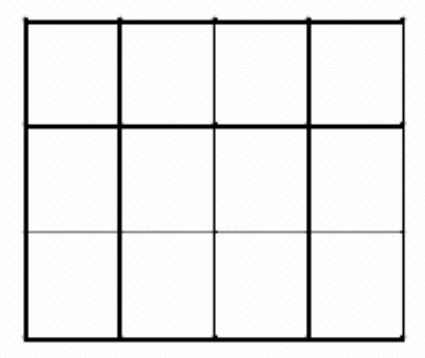

(b)

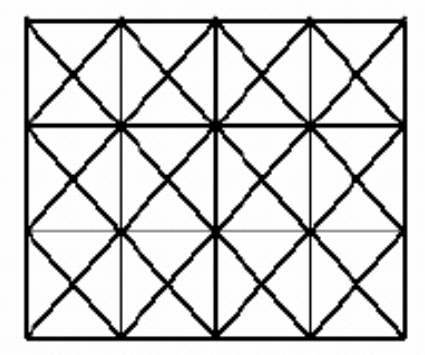

(c)

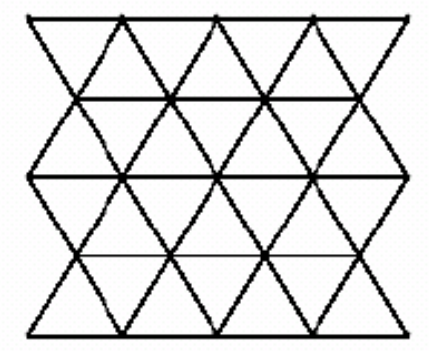

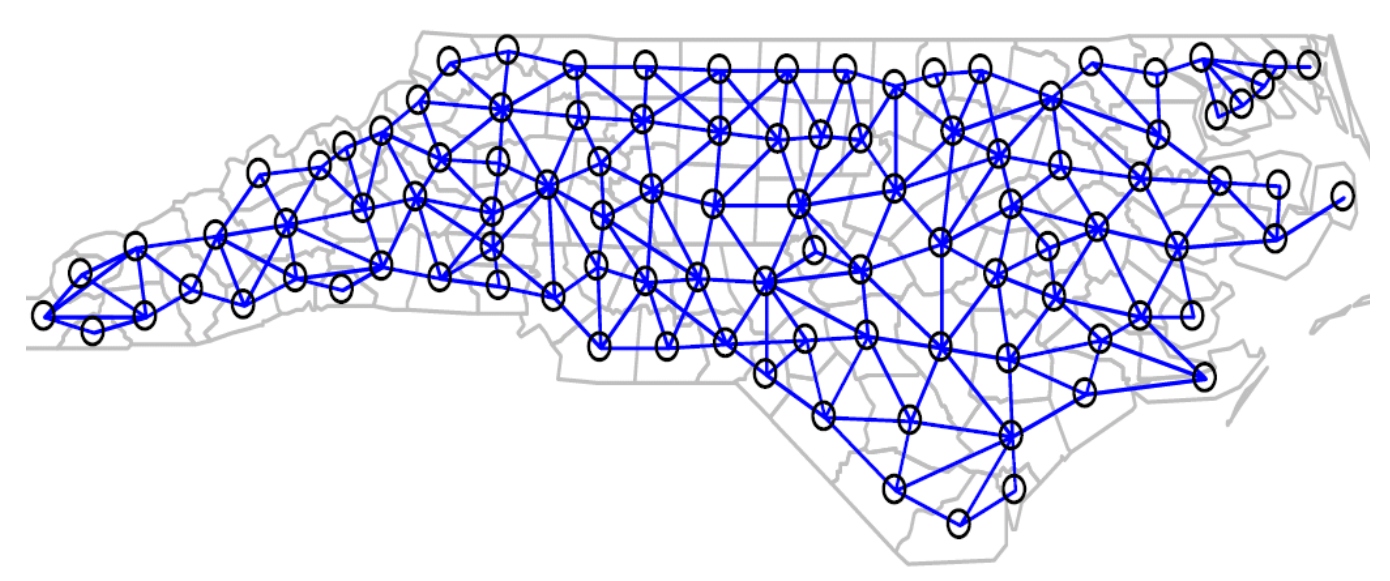

Atelier Spatial RASMA

Saint Louis du Sénégal 


\section{Champ de Markov sur $S$ fini}

- S réseau fini (régulier ou non)

- Loi de X caractérisée par ses conditionnelles

- Loi conditionnelle est « locale »

- Espace d'état $E$ général : binaire, fini, $N, R+, R, R^{* *} p$, mixte (gris $x$ variété), etc

$$
\pi_{A}\left(x_{A} \mid x^{A}\right)=\pi_{A}\left(x_{A} \mid x_{\partial A}\right) .
$$




\section{Champ de Gibbs}

1. Potentiels réels $\Phi=\{\Phi(x(A))\}$ définis sur une famille de parties $A$ de $S$

2. $\Phi \rightarrow$ énergie $U(\Phi ; \Lambda)$ sur $\Lambda$ conditionnelle $\partial \Lambda$

3. S'assurer que $\exp \{U(\Phi ; \Lambda)\}$ intégrable (potentiel admissible)

4. Le champ de Gibbs est de log-densité conditionnelle à $\partial \wedge$ proportionnelle à $\exp \{U(\Phi ; \Lambda)\}$ 


\section{Energie et loi conditionnelles d'un champ de Gibbs}

Soit $\wedge$ une partie de $S, \partial \wedge$ son voisinage

$$
\begin{array}{ll}
U_{\Phi}(x(\Lambda) \quad & x(\partial \Lambda))=\sum_{A: A \cap \Lambda \neq \emptyset} \Phi_{A}(x(A)) \\
& \text { et loi conditionnelle } \\
\pi(x(\Lambda) \quad \mid & x(\partial \Lambda))=Z^{-1}(x(\partial \Lambda)) \times \exp U_{\Phi}(x(\Lambda) \mid x(\partial \Lambda))
\end{array}
$$




\section{Spécification de Gibbs}

- Famille des parties $A$ sur lesquelles sont définit les potentiels

- Les potentiels $\Phi(A)$

- Vérifier l'admissibilité de $\exp U(\Phi)$

- Exemple : famille exponentielle de potentiels de paramètre $\theta$

$\Phi_{A}(y)=\left\langle\theta, \phi_{A}(y)\right\rangle$ où $\phi_{A} \in \mathbb{R}^{p}$ connues, 


\section{Exemple de potentiels contribuant à une loi conditionnelle (loi aux 4 ppv)}

- Cliques à un point $\{x\}$

- À deux points

- Potentiels contribuants à $\pi(\Lambda / \partial \Lambda)$

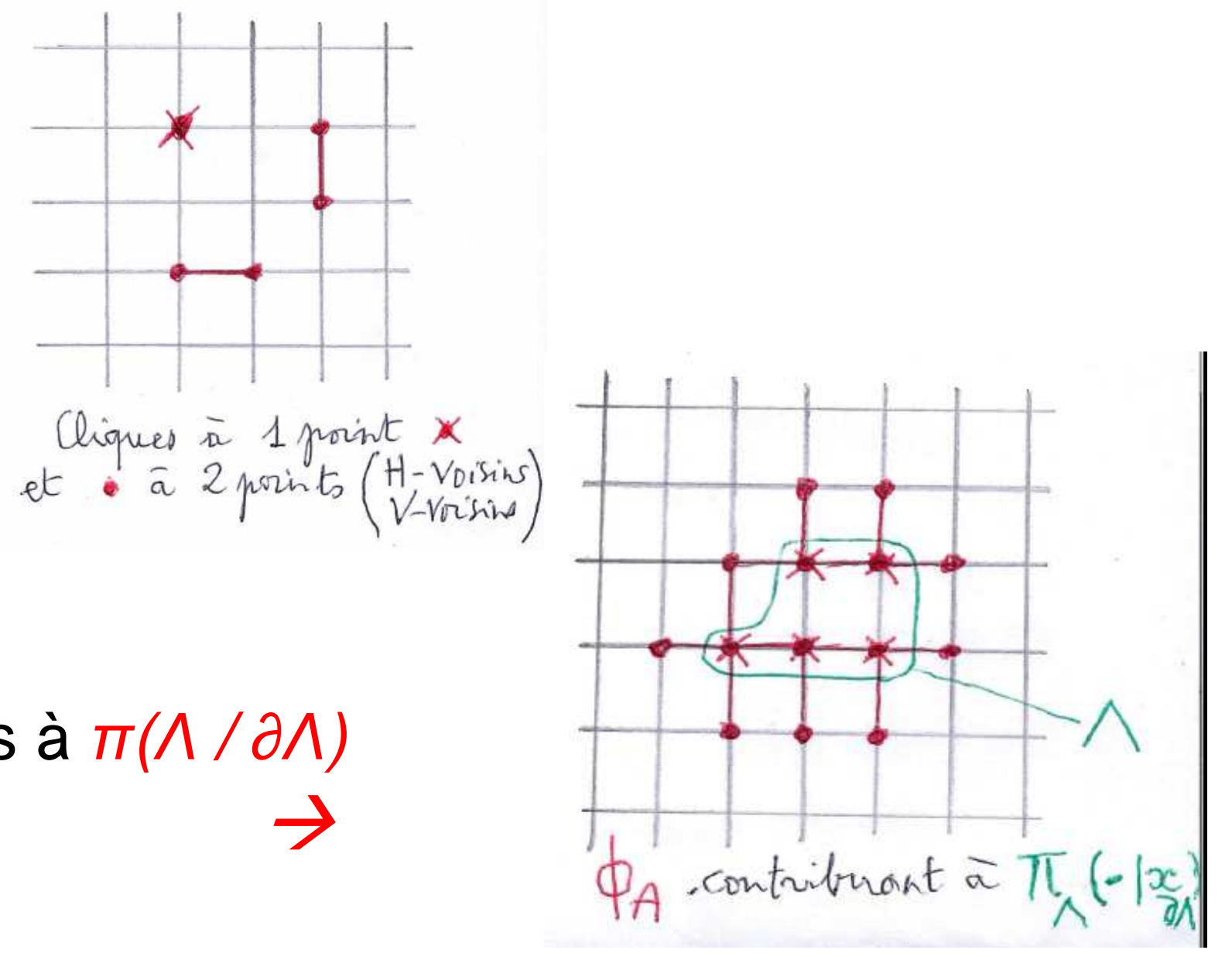




\section{Modèle d'lsing : état $E=\{-1,+1\}$ et sites$$
S=\{1,2, \ldots . ., n\}\}^{\star *} 2
$$

- Cliques : singletons et paires de ppv (plus proche voisin)

- Potentiels : $\Phi(x(i))=\alpha \cdot x(i)$ et $\Phi(x(i), x(j))=\beta \cdot x(i) \cdot x(j)$

- Energie : $U_{\Lambda}\left(x_{\Lambda} \mid x^{\Lambda}\right)=\alpha \sum_{i \in \Lambda} x_{i}+\beta \sum_{i \in \Lambda, j \in S:\langle i, j\rangle} x_{i} x_{j}$,

- loi conditionnelle en $i$ :

$$
\pi_{i}\left(x_{i} \mid x^{i}\right)=\pi\left(x_{i} \mid x^{i}\right)=\frac{\exp x_{i}\left(\alpha+\beta v_{i}(x)\right)}{2 \operatorname{ch}\left(\alpha+\beta v_{i}(x)\right)}
$$




\section{Généralisations du modèle d'Ising}

- Etats $\{0,1\}$ : présence - absence (écologie); sain - malade (épidémiologie)

- Plus d'états : niveaux de gris, $E$ fini ( $K$ variétés)

- Anisotropie : $\beta(H)$ pour horizontal, $\beta(V)$ pour vertical

- Non stationnaire : $\alpha(i)$ et $\beta(i, j)$ suivant les sites $i, j$

- Élargissement du voisinage $\partial i$ : i.e. aux 8 ppv

- Potentiels de triplets, quadruplets ....

- Simulations par MCMC (échantillonneur de Gibbs) 


\section{Exemples : 3 textures binaires aux 8 - ppv}

- Potentiels au plus de paires $\rightarrow 5$ paramètres

- Simulation par échantillonneur de Gibbs (Ants/nfields)

- Arrêt après 3000 itérations
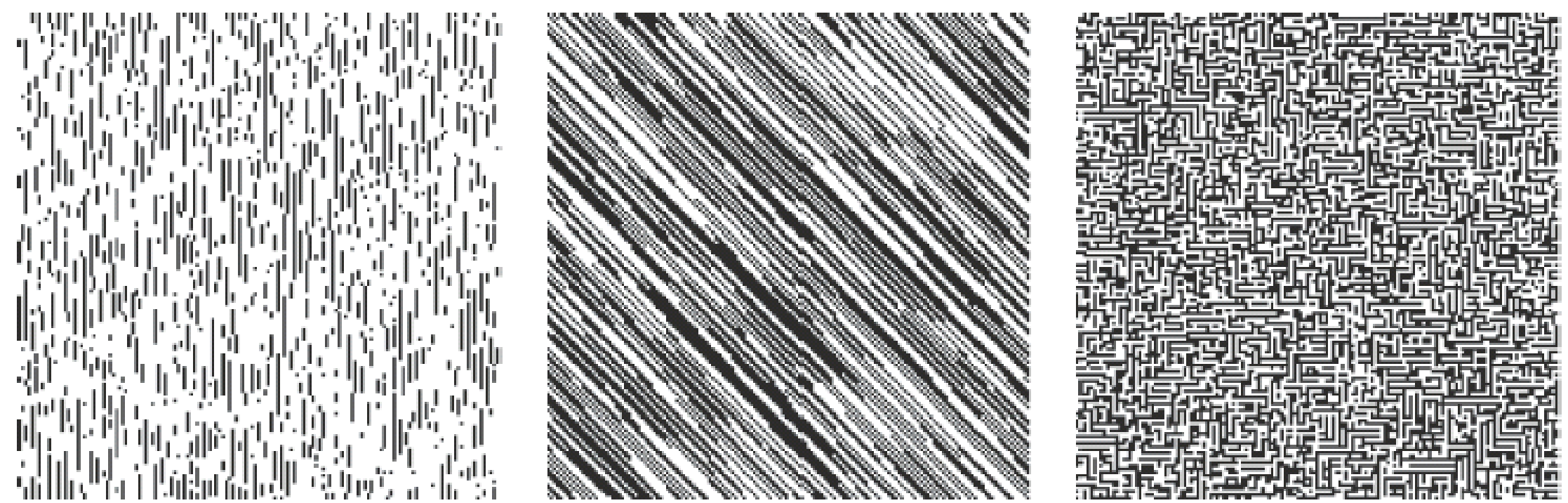

$$
\begin{array}{rrr}
0.0 & 1.0 & 0.0 \\
-1.0 & 2.0 & -1.0 \\
0.0 & 1.0 & 0.0
\end{array}
$$

$$
\begin{array}{rrr}
3.0 & 1.0 & 0.0 \\
-1.0 & 0.0 & -1.0 \\
0.0 & 1.0 & 3.0
\end{array}
$$$$
\begin{array}{lll}
-0.4 & 0.4-0.4
\end{array}
$$$$
\begin{array}{lll}
0.4 & 0.0 & 0.4
\end{array}
$$$$
-0.4 \quad 0.4-0.4
$$ 


\section{Modèle de Gibbs à nombre d'états fini (modèle de Potts)}

- Etats : $E=\{a(1), a(2), \ldots, a(K)\}, K$ états

- Potentiels : singletons et paires de sites voisins

$$
\begin{array}{ll}
\Phi_{\{i\}}(x)=\alpha_{k}, & \text { si } x_{i}=a_{k}, \\
\Phi_{\{i, j\}}(x)=\beta_{k, l}=\beta_{l, k}, & \text { si }\left\{x_{i}, x_{j}\right\}=\left\{a_{k}, a_{l}\right\}
\end{array}
$$

- Energie $: n(k)=n b$ sites modalité $k ; n(k, l)=n b$ de sites voisins de modalités $(k, l)$.

$$
U(x)=\sum_{k} \alpha_{k} n_{k}+\sum_{k<l} \beta_{k l} n_{k l} .
$$




\title{
Modèle de Potts échangeable
}

\author{
Très utile en traitement d'image
}

- Tous les états ont un comportement analogue c'est-à-dire : $\alpha(k)=\alpha$ et $\beta(k, l)=\beta$ pour tout $k, I$

- Dans ce cas il y a un seul paramètre de dépendance spatiale $\beta$ et la loi jointe vaut :

$$
\pi(x)=Z^{-1} \exp \{-\beta n(x)\}
$$




\section{Modèle échangeable à 3 états}

$\beta \uparrow$ augmente la régularité géométrique des plages constantes
(a) $\beta=0.5$
(b) $\beta=0.6$
(c) $\beta=0.7$

(Echantillonneur de Gibbs à 5000 itérations avec AntsInFields)

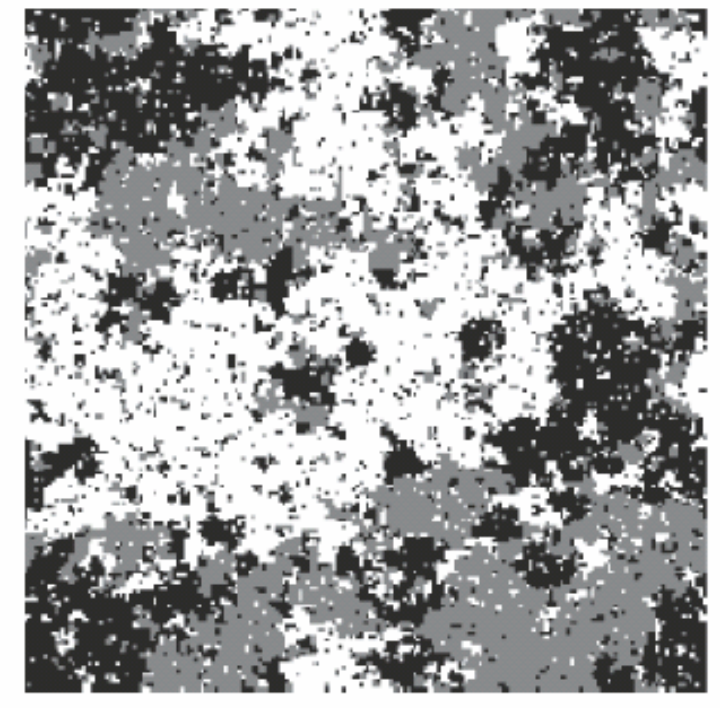

(a)

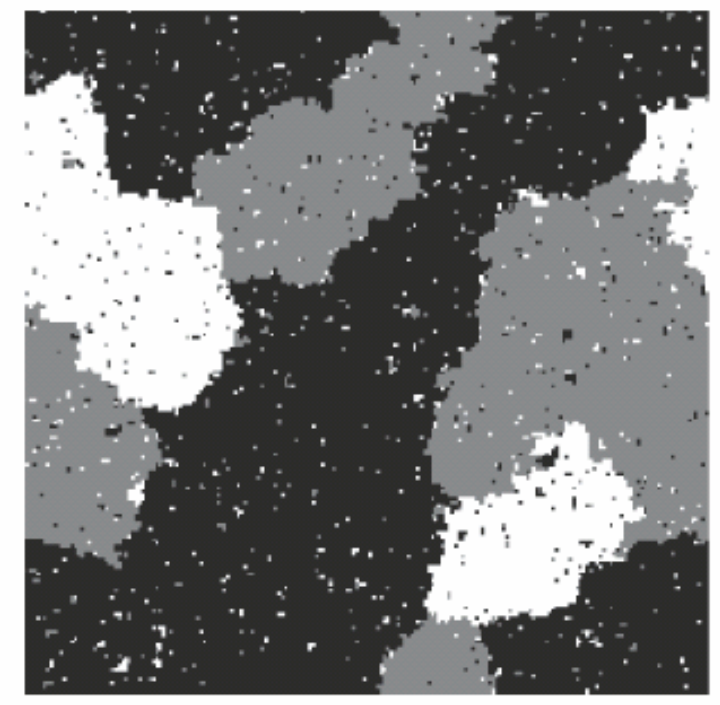

(b)

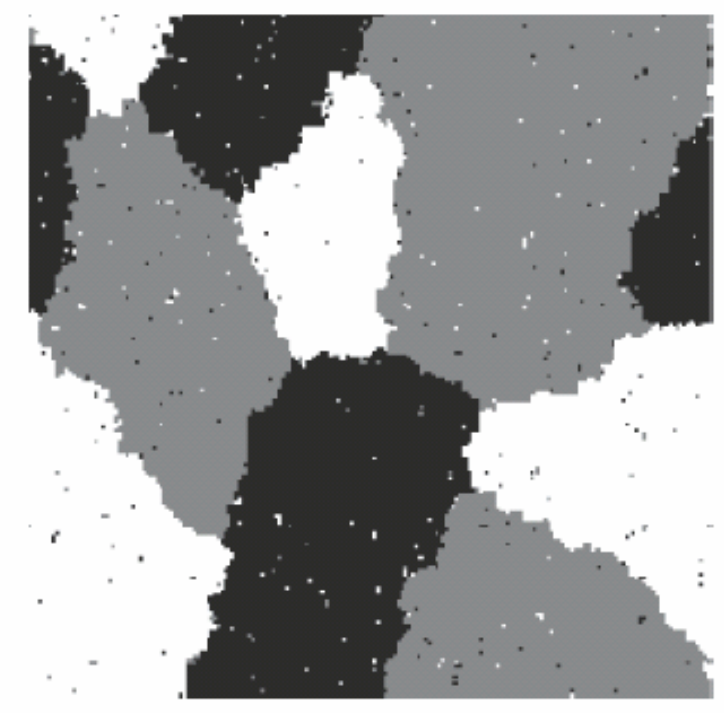

(c) 


\section{Un champ gaussien est un champ de Gibbs}

- Soit $X=N(\mu, \Sigma)$ de moyenne $\mu$ et covariance $\Sigma(Q$ inverse $\Sigma)$

- Alors X est de Gibbs avec :

$\rightarrow$ potentiels de singletons et de paires

$\rightarrow$ énergie $-U$

- Obtention des lois conditionnelles comme champ de Gibbs

$$
U(x)=\frac{1}{2}^{t}(x-\mu) Q(x-\mu),
$$

de potentiels de singletons $\Phi_{\{i\}}$ et de paires $\Phi_{\{i, j\}}$ :

$$
\Phi_{\{i\}}(x)=x_{i} \sum_{j: j \neq i} q_{i j} \mu_{j}-\frac{1}{2} q_{i i} x_{i}^{2} \text { et } \Phi_{\{i, j\}}(x)=-q_{i j} x_{i} x_{j} \text { si } i \neq j .
$$




\section{Simulation d'un champ de Markov}




\section{Simulation par dynamique de chaîne de Markov}

(MCMC pour Monte Carlo Markov Chain)

- Objectif : simuler une loi $\Pi$ sur $E$

- Principe MCMC : construire une chaîne de Markov $(X(n)$, $n>0)$ sur $E$ de transition $P(x, \bullet)$ t.q. :

$1-P$ est irréductible (tous les états communiquent),

$2-P$ est $\Pi$ - invariante $(\Pi P=\Pi)$

$3-P$ est apériodique

Propriété : Si (1-2-3), la loi de $X(n)$ tend vers $\Pi$ 


\section{Irréductible, invariance et apériodicité}

- Irréductible : la chaîne $P$ fait communiquer tous les états de $E$ entre eux

- $\Pi$ - invariante $(\Pi P=\Pi)$ : si la loi de $X(n)$ est $\Pi$, celle de $X(n+1)$ est encore $\Pi$ ( $\Pi$ est la loi invariante de la chaîne)

- Périodique : si il existe une partition $\{E(1), E(2), \ldots, E(k)\}$ de $E$ t.q. la chaîne circule successivement dans $E(1) \rightarrow E(2) \rightarrow \ldots E(k) \rightarrow E(1)$ etc

- Apériodique : si non périodique 


\section{Comment construire une telle chaîne ?}

- Apériodicité et irréductibilité se vérifient au cas par cas

- Pas facile de trouver $P$ qui soit $\Pi$ - invariante : $\Pi$ est vecteur propre de $P$ associée à la valeur propre 1 !

- Condition suffisante assurant la $\Pi$ - invariance de $P$ : la $\Pi$ - réversibilité :

pour tout $x, y: \Pi(x) P(x, y)=\Pi(y) P(y, x)$ 


\section{Deux algorithmes markoviens classiques}

- Echantillonneur de Gibbs sur un espace produit $E^{* *} S$ : $S=\{1,2, \ldots, n\}$ ensemble des sites, état $E$ en chaque site

- L’algorithme de Metropolis (espace E général)

Pour l'un et l'autre, la transition $\mathrm{P}$, est, par construction, $\Pi-$ réversible, donc $\sqcap-$ invariante

$\rightarrow$ simulateur MCMC de $\Pi$ si on vérifie de plus que $P$ est apériodique et irréductible 


\section{Simulation par échantillonneur de Gibbs d'une loi $\pi$ sur $E^{* *} S$}

- Loi $\pi$ sur espace produit $E^{* *} S$

- Connaître les lois conditionnelles en tout $i: \pi_{i}\left(y_{i} \mid x^{i}\right)$

- Relaxation « site par site» sur $S$ suivant la conditionnelle :

$$
P_{i}(x, y)=\pi_{i}\left(y_{i} \mid x^{i}\right) 1\left(x^{i}=y^{i}\right)
$$

- Un «balayage» de $S$ : itérer de $i=1, n \rightarrow$ la transition $P$

- Itérer les balayages

- Propriété : P est $\pi$-réversible.

Donc l'échantillonneur de Gibbs simule approximativement $\pi$ Après un grand nombre de balayages de $S$. 


\section{Transition pour un balayage de $S$}

- Visite séquentielle de $S: 1 \rightarrow 2 \rightarrow 3 \rightarrow \ldots \rightarrow n$

- Au i-ème pas, relaxation au site $i$

- Enchaînement sur un balayage donne la transition :

$$
P_{S}(x, y)=\prod_{i=1}^{n} \pi_{i}\left(y_{i} \mid y_{1}, \ldots, y_{i-1}, x_{i+1}, x_{i+2}, \ldots, x_{n}\right)
$$




\section{La transition $P$ de l'algorithme de Métropolis}

- $\Pi$ loi sur espace d'état $E$ général, $x, y$ deux états

Construction de $P$ en deux étapes :

1 - Proposition de changement $x \rightarrow y$ suivant une transition $Q(x, y)$ symétrique ( $Q$ est la proposition de changement)

2 - Acceptation du changement avec une probabilité $a(x, y)$

Propriété : si $a(x, y)=\min \{1, \Pi(y) / \Pi(x)\}$, alors $P$ est $\Pi-$ réversible (donc $\Pi$ - invariante)

Si de plus $\mathrm{P}$ est irréductibilité et apériodique, on a un autre algorithme de simulation de $\Pi$ 


\section{Algorithme de Métropolis}

1 - x état initial. Changement $x \rightarrow y$ suivant $Q(x, y)$

2 - Si $\Pi(y) \geq \Pi(x)$, garder $y$.

3 - Sinon, tirer $U$ uniforme sur $[0,1]$ :

(a) si $U>p=\Pi(y) / \Pi(x)$, garder $x$.

(b) Sinon, garder $y$.

4 - Revenir en (1)

L'algorithme de Métropolis - Hastings correspond à une proposition de changement $Q$ non symétrique.

Remarque importante : il suffit de connaître $\Pi$ à un facteur près pour construire cet algorithme (le cas pour $\Pi$ de Gibbs). 


\section{Exemple : simulation d'un Ising isotropique aux 4-ppv}

(I) - Par échantillonneur de Gibbs

Les lois conditionnelles en chaque site $i$ sont explicites en terme de $v(i)$, la somme aux 4-ppv

$$
\begin{aligned}
& \pi(x)=Z^{-1} \exp \left\{\alpha \sum_{i} x_{i}+\beta \sum_{<i, j>} x_{i} x_{j}\right\}, \\
& \pi_{i}\left(x_{i} \mid x^{i}\right)=\frac{\exp x_{i}\left(\alpha+\beta v_{i}\right)}{2 \operatorname{ch}\left(\alpha+\beta v_{i}\right)} .
\end{aligned}
$$




\section{AntsInFields}

«Boite noire » illustrant le livre de G. Winkler (Springer, 2002) :

"Image Analysis, random fields and dynamic MC Methods »

Largement buggée et non ouverte à la programmation.

Outil de démonstration, sur des thèmes d'analyse d'image et de statistique de champ de Gibbs.

\section{Exemple : $\S 3-2$}

$\rightarrow$ simulation Ising isotropique aux 4-ppv par échantillonneur de Gibbs $h=0, b=0,0.2$ et 0.4 . Voir petit à petit se former des plages

$\rightarrow$ simulation d'un modèle de Potts à 4 niveaux de gris, paramètres : nombres de classes, $h$ et $b$ et distance retenue entre les configurations voisines 


\section{Simulation d'un Ising (suite)}

\section{(II) - Métropolis par échange de spins}

1 - on tire au hasard 2 sites $i$ et $j$ et on permute les spins $x$ (i) et $x(j)$ : on passe ainsi de $x \rightarrow y$ avec une probabilité de transition $Q(x, y)$

2 - le quotient $\Pi(x) / \Pi(y)$ s'explicite facilement en fonction de $x(i), x(j), v(i)$ et $v(j)$ (cf. poly)

3 - Mettre en œuvre Métropolis.

$$
Q(x, y)= \begin{cases}\frac{2}{n^{2}\left(n^{2}-1\right)} & \text { pour un tel échange } \\ 0 & \text { sinon }\end{cases}
$$




\section{Champ de Markov et champ de Gibbs}

- $S=\{1,2 ; 3, \ldots, n\}$ et $G$ graphe symétrique sur $S$

- $\quad<i, j>$ : $i$ et $j$ voisins pour $G$.

- $\partial A=$ voisinage de $A$ pour $G$

Clique de $G$ : singletons + parties $A$ t.q. les points de $A$ sont tous voisins

- $\quad C(G)=$ toutes les cliques de $G$

Champ de Markov:

$$
\pi_{A}\left(x_{A} \mid x^{A}\right)=\pi_{A}\left(x_{A} \mid x_{\partial A}\right) .
$$




\section{Le théorème de Hammersley - Clifford Gibbs $\equiv$ Markov}

- $\pi$ un champ de Markov pour graphe $G$

- Positivité : pour tout $x, \pi(x)>0$

- Propriété $(\mathrm{H}-\mathrm{C})$ : alors $\pi$ est- un champ de Gibbs dont les potentiels sont limités aux cliques de $G$

- Réciproque : tout champ de Gibbs est un champ de Markov pour le graphe engendré par les potentiels de Gibbs. 


\section{Recollement de lois conditionnelles}

- Objectif : définir un modèle à partir de ses spécifications locales

- En général, des spécifications locales «ne se recollent pas »

- E sous ensemble réel

- Les spécifications de la « famille exponentielle » ci-dessous se recollent.

Résultat : si pour tout $i$

$$
\begin{aligned}
& \text { Recollement des } \pi\left(x_{i} / x_{\partial i}\right) \\
\log \pi\left(x_{i} / x_{\partial i}\right) & =A_{i}\left(x_{\partial i}\right) B\left(x_{i}\right)+C\left({ }_{i}\left(x_{i}\right)+D_{i}\left(x_{\partial i}\right)\right. \\
\text { où } B_{i}(0) & = \\
& C_{i}(0)=0 \text { et } A_{i}\left(x_{\partial i}\right)=\alpha_{i}+\sum_{j \in \partial i} \beta_{i j} x_{j}, \text { et } \beta_{i j}=\beta_{j i}, \\
\text { Alors } & \Longrightarrow \pi \text { est un champ de Gibbs de potentiels : } \\
\Phi_{i}\left(x_{i}\right) & =\alpha_{i} B_{i}\left(x_{i}\right)+C_{i}\left(x_{i}\right) \text { et } \Phi_{i, j}\left(x_{i}, x_{j}\right)=\beta_{i j} B_{i}\left(x_{i}\right) B_{j}\left(x_{j}\right) .
\end{aligned}
$$




\section{Auto - modèle de Besag (1974)}

- E sous ensemble de $R$

- Famille exponentielle du type précédent Alors les lois conditionnelles se recollent en la loi de Gibbs $\pi$ :

$$
\pi(x)=Z^{-1} \exp \left\{\sum_{i \in S} \Phi_{i}\left(x_{i}\right)+\sum_{\langle i ; j\rangle} \beta_{i j} x_{i} x_{j}\right\}
$$




\section{Auto-modèle de Markov : auto - régression pour espace $E$ général}

- $E=\{0,1\}$ : auto-logistique (états binaires)

- $E=\{0,1,2, \ldots, K\}:$ auto-binomial

- $E=N$ : auto-poisson (comptage en épidémiologie)

- $E=R+$ : auto-exponentiel (Gamma), pluviométrie

- $E=R\left(R^{* *} d\right)$ : auto-gaussien

- Possibilité d'ajouter des covariables explicatives 


\section{Modèle Auto-Logistique : $E=\{0,1\}$}

- paramètre $\theta(x(i))$ et loi Logit conditionnel

$$
\begin{aligned}
\theta_{i}\left(x_{i}\right) & =\left\{\alpha_{i}+\sum_{j:\langle i, j\rangle} \beta_{i j} x_{j}\right\}, \\
\pi_{i}\left(x_{i} \mid x^{i}\right) & =\frac{\exp x_{i}\left\{\alpha_{i}+\sum_{j:\langle i, j\rangle} \beta_{i j} x_{j}\right\}}{1+\exp \left\{\alpha_{i}+\sum_{j:\langle i, j\rangle} \beta_{i j} x_{j}\right\}} .
\end{aligned}
$$

- L'énergie jointe du champ de Gibbs est

$$
U(x)=\sum_{i} \alpha_{i} x_{i}+\sum_{\langle i, j\rangle} \beta_{i j} x_{i} x_{j} .
$$

- Généralisation à l'auto-binomial 


\section{Auto - modèle de Poisson : $E=N$ (comptage en épidémiologie, etc)}

- la loi conditionnelle Poisson suit un MLG

- admissibilité : $\beta<0$ (compétition)

- coopération possible en bornant $E$ à $K<\infty$

$$
\begin{aligned}
\text { Si } \forall i & \in S: \pi\left(x_{i} \mid x_{\partial i}\right)=\mathcal{P}\left(\lambda_{i}\left(x_{\partial i}\right),\right. \text { un MLG : } \\
\log \left(\lambda_{i}\left(x_{\partial i}\right)\right. & =\alpha_{i}+\sum_{j \in \partial i} \beta_{i j} x_{j} \text { où } \alpha_{i}+\sum_{j \in \partial i} \beta_{i j} x_{j}=\beta_{j i} .
\end{aligned}
$$

Admissibilité : $\forall i, j, \beta_{i j}<0$ (compétition).

Alors $\pi$ est d'énergie jointe

$$
U(x)=\sum_{i \in S} \alpha_{i} x_{i}+\log \left(x_{i} !\right)+\sum_{\langle i, j\rangle} \beta_{i j} x_{i} x_{j}
$$




\section{Auto - exponentiel : $E=\boldsymbol{R}+$}

(variable $>0$ : pluviométrie, etc)

- loi conditionnelle exponentielle suit un MLG

- admissibilité : $\beta<0$ (compétition)

- coopération possible en bornant $E$ à à $K<\infty$

$$
\begin{array}{rll}
\text { Auto-Exponentiel } & : \text { si } & \forall i \in S: \pi\left(x_{i} \mid x_{\partial i}\right)=\mathcal{E} \operatorname{xp}\left(\mu_{i}\left(x_{\partial i}\right),\right. \text { un MLG: } \\
\log \left(\mu_{i}\left(x_{\partial i}\right)\right. & = & \alpha_{i}+\sum_{j \in \partial i} \beta_{i j} x_{j} \text { où } \alpha_{i}+\sum_{j \in \partial i} \beta_{i j} x_{j}=\beta_{j i} . \\
\text { Admissibilité : } \quad \forall i, j, \alpha_{i}> & 0 \text { et } \beta_{i j} \geq 0 \text { (compétition). } \\
& \text { Alors } \pi \text { est d'énergie jointe } \\
U(x) \quad=\quad & -\left\{\sum_{i \in S} \alpha_{i} x_{i}+\sum_{\langle i, j\rangle} \beta_{i j} x_{i} x_{j}\right\}
\end{array}
$$




\section{Auto - modèle avec covariables $z$}

- Il y a trop de paramètres $\alpha$ et $\beta$ si le modèle est non stationnaire

- Modéliser les $\alpha, \beta$ à partir de covariables $z$ : Exemple :

$\begin{aligned} \text { Poids connus } & :\left(a_{i}\right),\left(w_{i j}\right) \text { symétrique } \\ \text { Covariable } & : \quad z=\left(z_{i}\right), z_{i} \in \mathbb{R}^{p} \text { observable sur } S \\ \beta_{i j}= & \delta w_{i j} \text { et } \alpha_{i}=a_{i} \times{ }^{t} \gamma z_{i} \\ & \Longrightarrow \text { modèle à }(p+1) \text { paramètres. }\end{aligned}$ 


\title{
Estimation d'un champ de Markov
}

\author{
3 procédures
}

- Max de Vraisemblance : efficace, difficile (cste de normalisation) $\rightarrow$ méthodes numériques MCMC

- Pseudo Vraisemblance Conditionnelle (PVC) : facile à mettre en place, bonnes propriétés, proche MV si peu de dépendance spatiale.

- Codage : facile, moins efficace, test de chi2 direct 


\section{Maximum de vraisemblance sur $D(n)$}

Vraisemblance conditionnelle à $x\left(\partial D_{n}\right)$ :

$\pi_{n}\left(x\left(D_{n}\right) / x\left(\partial D_{n}\right) ; \theta\right)=Z^{-1}\left(\partial D_{n} ; \theta\right) \exp U\left(x\left(D_{n}\right) \mid x\left(\partial D_{n}\right) ; \theta\right)$

où $Z$, la constante de normalisation, vaut (cas $E$ fini) :

$Z\left(\partial D_{n} ; \theta\right)=\sum_{y\left(D_{n}\right)} \exp U\left(y\left(D_{n}\right) \mid x\left(\partial D_{n}\right) ; \theta\right)$.

- L'estimation du MV est convergente si $\pi$ appartient à famille exponentielle invariante par translation $\left(S=Z^{* *} 2\right)$

- Difficulté de calcul de la constante de normalisation $Z$ $\rightarrow$ calcul de $Z$ par MCMC ou par algorithme de score 


\section{Pseudo - Vraisemblance Conditionnelle (PVC - Besag : 1974)}

$$
P V C_{n}(X)=\prod_{i \in D_{n}} \pi_{i}\left(x_{i} \mid x_{\partial i} ; \theta\right)
$$

Exemple auto-logistique isotropique aux 4 -ppv:

$$
P V C_{n}(x)=\prod_{i \in D_{n}} \frac{\exp x_{i}\left(\alpha+\beta \sum_{j:\langle i, j\rangle} x_{j}\right)}{1+\exp \left(\alpha+\beta \sum_{j:\langle i, j\rangle} x_{j}\right)}
$$

- $\mathrm{PVC}=$ produit en chaque site $i$ des probabilités conditionnelles

- Bonne fonctionnelle d'estimation (convergence, normalité sous des hypothèses raisonnables)

- Pour un CAR gaussien $\equiv$ MCO sur les résidus

- Obtention estimation via logiciel dédié aux MLG ......

- Attention : calcul spécifique de la variance d'estimation 


\section{Influence du taux de nitrate des eaux sur la mortalité par cancer, Valence - Espagne}

(Ferrandiz et al. Biometrics - 1995)

Auto modèle poissonnien $(\mathrm{Y}(\mathrm{i})$ variable de comptage);

Covariables : $\mathrm{x}(1)=\%$ population $>40$ ans; et $\mathrm{x}(2)=\%$ nitrate dans l'eau. Matrice de poids : fonction des populations $\mathrm{u}(\mathrm{i})$ et des distances $\mathrm{d}(\mathrm{i}, \mathrm{j})$;

$$
\begin{gathered}
\langle i, j\rangle \text { si } a_{i j}=\frac{\sqrt{u_{i} \times u_{j}}}{d_{i j}}>a \text { et } \gamma_{i j}=\gamma \times a_{i j} \\
\log \left(\lambda_{i}\right)=\alpha+\log \left(u_{i}\right)+\beta_{1} x_{i 1}+\beta_{2} x_{i 2}+\gamma \sum_{j:\langle i, j\rangle} a_{i, j} y_{j}
\end{gathered}
$$




\section{Influence du taux de nitrate dans les eaux sur la mortalité par cancer (suite)}

$$
\log \left(\lambda_{i}\right)=\alpha+\log \left(u_{i}\right)+\beta_{1} x_{i 1}+\beta_{2} x_{i 2}+\gamma \sum_{j:\langle i, j\rangle} a_{i, j} y_{j}
$$

\begin{tabular}{|r||l|l|l|l||l}
\hline Modèle & $\alpha$ & $\gamma$ & $\beta_{1}$ & $\beta_{2}$ & $\chi^{2}$ \\
\hline \hline Constant & -7.3 & $* * * * *$ & $* * * * *$ & $* * * * *$ & 483.1 \\
\hline \hline AR-Poisson : PVC & -7.76 & $-6.2810^{-9}$ & $* * * * *$ & $* * * * *$ & 384.2 \\
\hline \hline MV & -7.76 & $-6.5210^{-9}$ & $* * * * *$ & $* * * * *$ & 363.2 \\
\hline \hline Régression & -8.91 & $* * * * *$ & 2.96 & $-1.9610^{-3}$ & 323.2 \\
\hline \hline Complet : PVC & -8.776 & $-2.8310^{-9}$ & 2.69 & $-2.1510^{-3}$ & 333.0 \\
\hline MV & -8771 & $-2.8010^{-3}$ & 2.67 & $-2.1710^{-3}$ & 309.1 \\
\hline
\end{tabular}




\section{Estimation et simulation de $\mathbf{3}$ textures binaires:}

(a) cailloux; (b) liège, (c) rideau (Cross et Jain)

- 3 textures binaires réelles de taille $64 \times 64$

$\rightarrow$

Estimation par PVC de 3 modèle auto-logistique

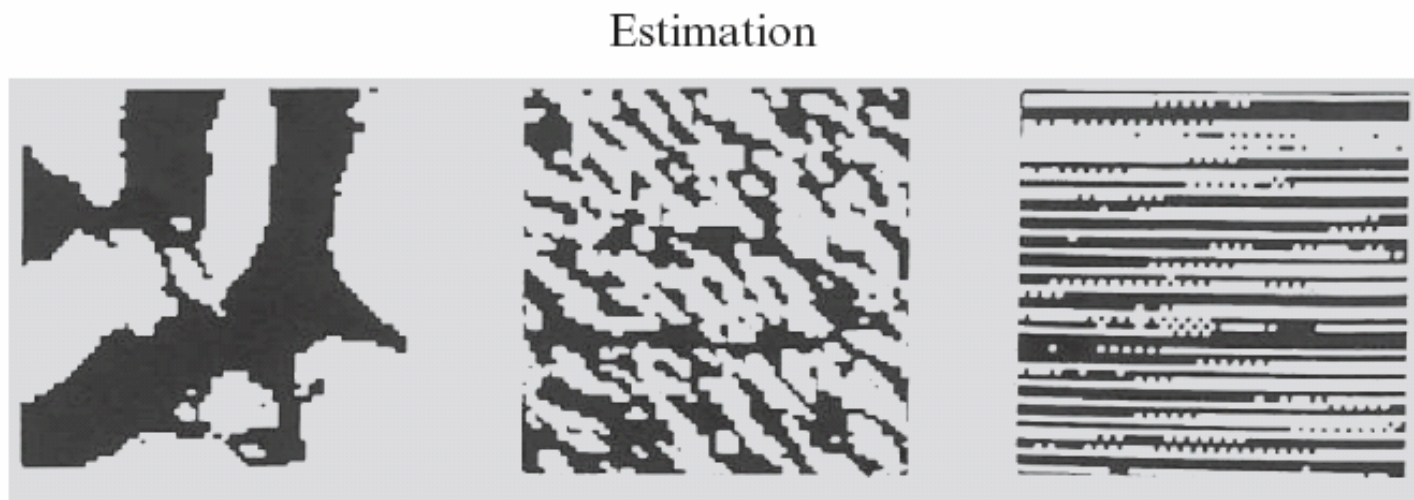

- Simulation des textures $\rightarrow$ estimées (éch. de Gibbs)

- Bilan : bonne adaptation de: (1) la modélisation Markov,

(2) l'estimation par PVC et

(3) la simulation par ech. de

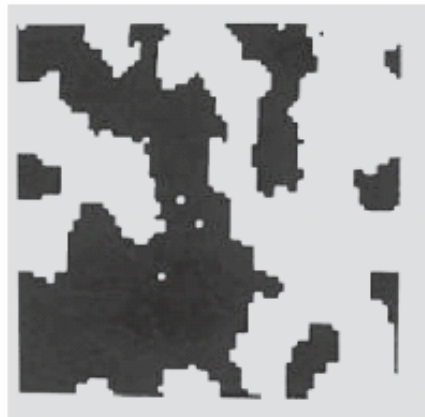

(a)

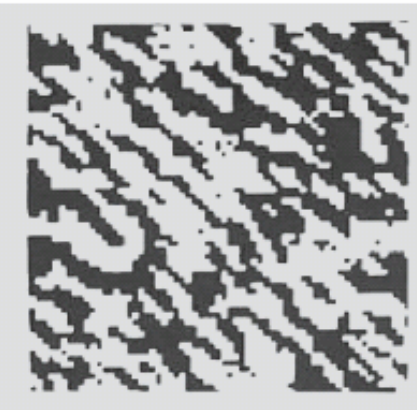

(b)

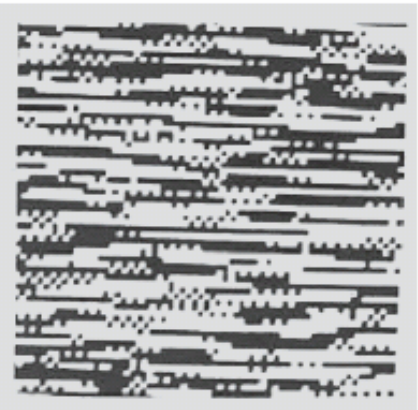

(c) Gibbs. 


\section{$C$ « Codage » de $S$}

Définition : $C$ est un codage de $S$ si 2 sites $s \neq t$ de $C$ ne sont jamais voisins

- Ex 1 : les $x$ rouges pour la relation aux 4 ppv $\rightarrow$

- Ex 2 : les $\square$ verts pour la relation aux 8 ppv $\rightarrow$
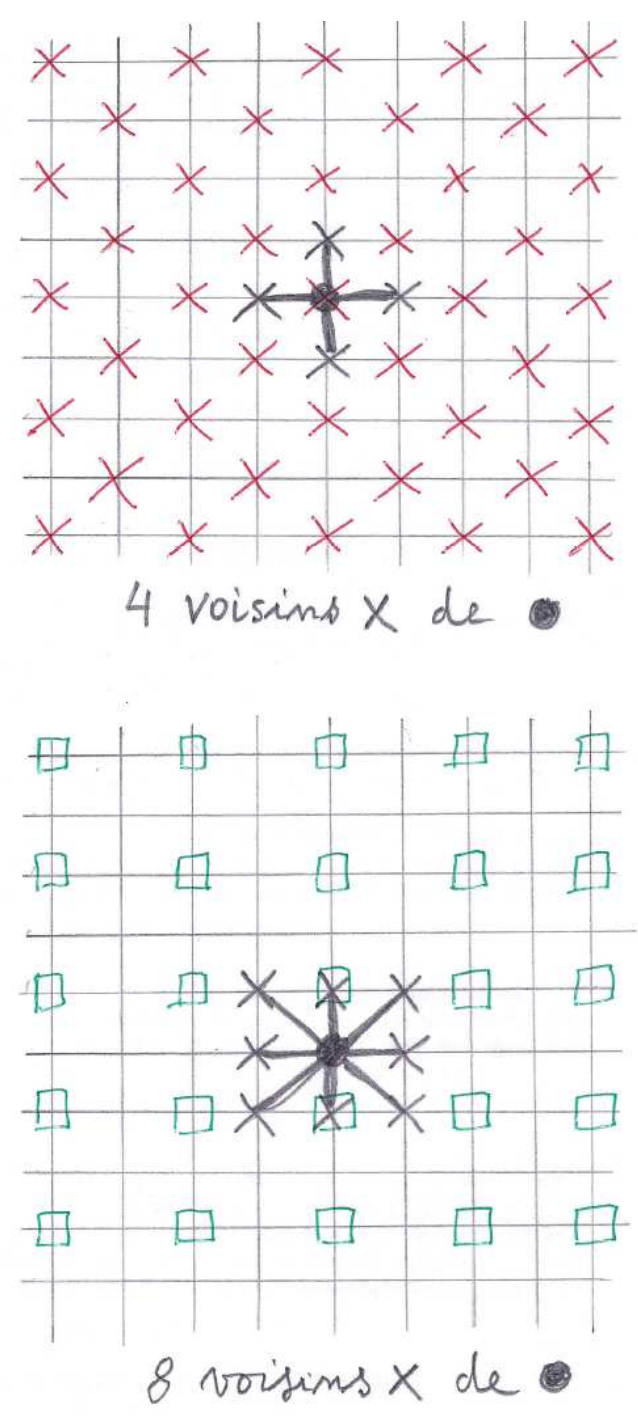


\section{Estimation par $C$ - codage}

- Soit $C$ un ensemble de codage de $S$

- La propriété fondamentale : indépendance des $X(s)$, s dans $C$, conditionnellement aux $x(S \mid C)$ extérieurs

- La vraisemblance sur $C$ conditionnelle aux $x(S \mid C)$ est (exactement) le produit des lois conditionnelles

$$
\begin{aligned}
L_{C}(x(C) \mid x(S \backslash C))= & \prod_{i \in C} \pi_{i}\left(x_{i} \mid x(S \backslash C)\right) \\
& =\prod_{i \in C} \pi_{i}\left(x_{i} \mid x_{\partial i}\right)
\end{aligned}
$$




\section{Conséquences}

- Même propriétés que pour un estimateur du MV de variables indépendantes (i.n.i.d.)

- Normalité, test du chi2 d'une sous hypothèse

- Calcul de l'estimation et de la variance d'estimation avec un logiciel dédié aux MLG

- Plusieurs choix d'ensemble de codage possible, mais les estimateurs associés sont dépendants 


\section{Modèle de répartition spatiale d'une espèce végétale (présence / absence) \\ Variété : la grande laîche}

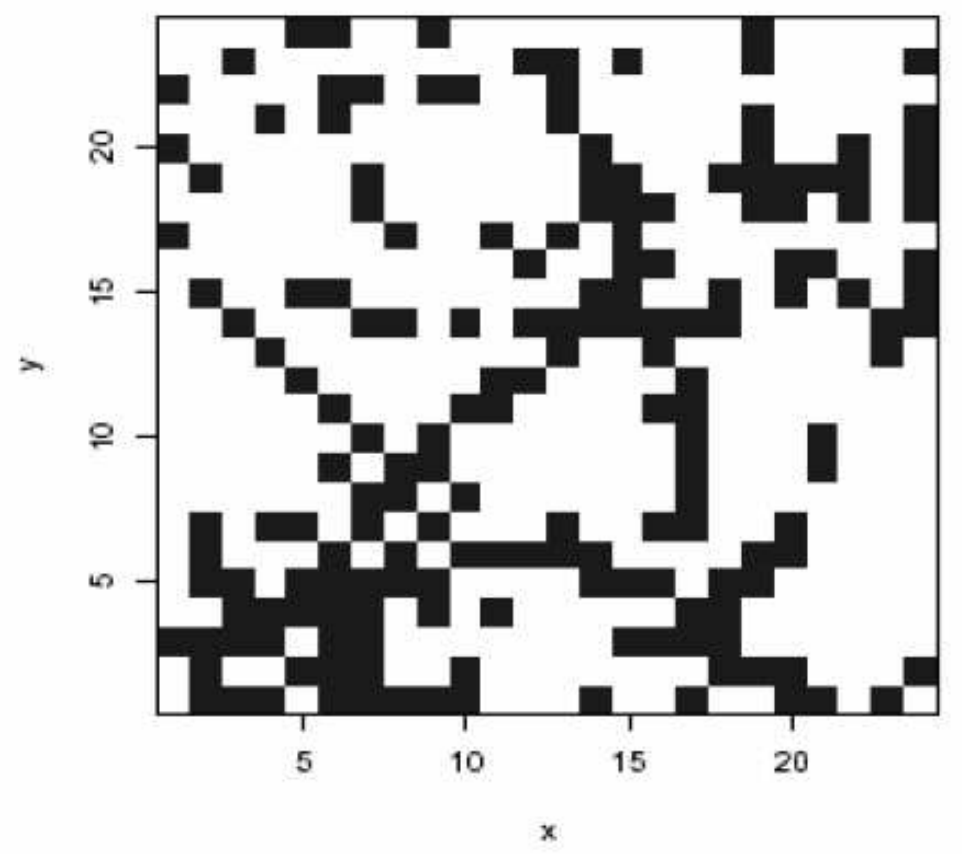

(a) Présence (ם) ou absence ( $\square$ ) de grande laîche. 


\section{2 modèles auto - logistiques :}

(I) aux 4-ppv (v(1)) - (II) aux 8 - ppv (v(2))

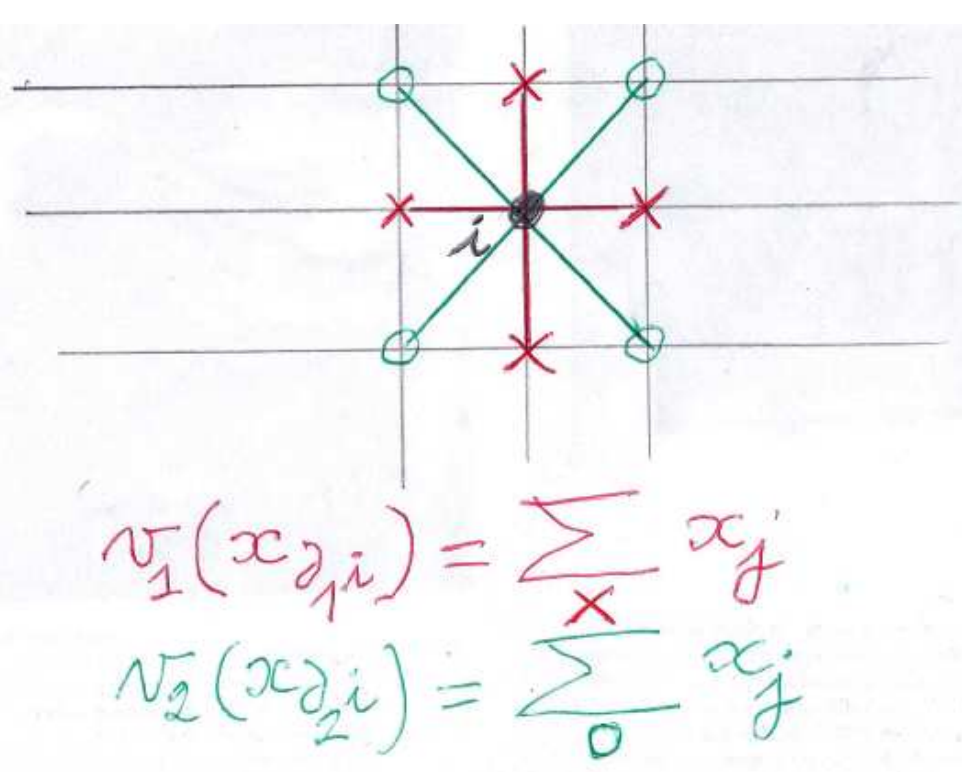

$$
P\left(X_{i}=1 \mid x_{j}, j \neq i\right)=\frac{\exp V_{i}(x)}{1+\exp V_{i}(x)} \text { où }
$$

(I) : $V_{i}(x)=\beta_{0}+\beta_{1} v_{1}\left(x_{\partial_{1} i}\right)$, où $v_{1}\left(x_{\partial_{1} i}\right)$ est la somme des 4 p.p.v.

(II) $: V_{i}(x)=\beta_{0}+\beta_{1} v_{1}\left(x_{\partial_{1} i}\right)+\beta_{2} v_{2}\left(x_{\partial_{2} i}\right)$,

$v_{2}\left(x_{\partial_{2} i}\right)$ est la somme des 4 voisins à distance $\sqrt{2}$ (voisins diagonaux) 


\section{Résultats : estimations MV, PMV et codage}

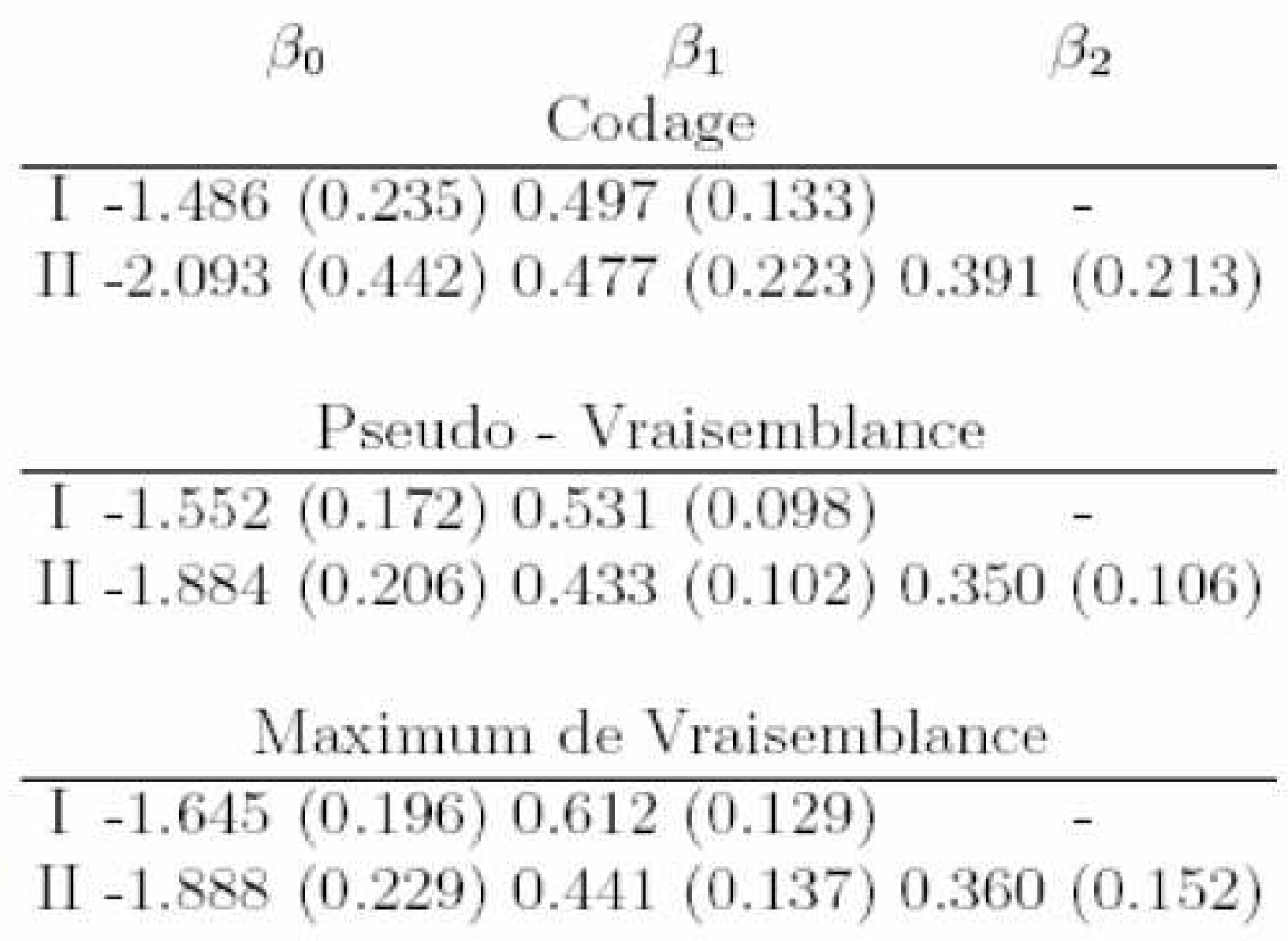




\section{Logiciel pour les champs de Markov}

- $\boldsymbol{R}$ ne fait pas grand-chose en dehors des champs gaussiens.

- Simulation de textures générales : AntsInFields (Winkler), logiciel gratuit téléchargeable.

- Pour l'estimation par PVC ou par codage, on peut utiliser un logiciel dédié aux modèles linéaires généralisés : ils donneront la bonne estimation.

Attention : si la variance donnée est correcte pour l'estimation par codage, elle ne l'est plus pour l'estimation par PVC.

La variance de l'estimation par PVC doit se calculer analytiquement ou par Monte Carlo à partir de la formule adéquate. 


\section{(II) - Modèle AR spatial}




\section{(II) - Modèle AR spatial}

- L'espace d'état est $E=\mathbf{R}$ ou $\mathbf{R}^{* *} \mathbf{p}$

- Le modèle est définit par un ensemble d'équations " spatiales » simultanées (comme en économétrie) en référence à un graphe d'influence

- Le bruit de modèle est blanc (SAR) ou coloré (CAR)

- Souvent le modèle est supposé gaussien 


\section{Ex 1 : la mort subite du nourisson}

(données sids de spdep, Cressie et al)

- $\mathrm{X}(\mathrm{s})=$ taux de sids pour le canton s (sudden infant deaths in north carolina 74-78)

- Régions et graphe de voisinage

Covariables :

- Nombre de naissances,

- Pourcentage de bébés de chaque communauté,

- Variables socio-économiques, etc ...

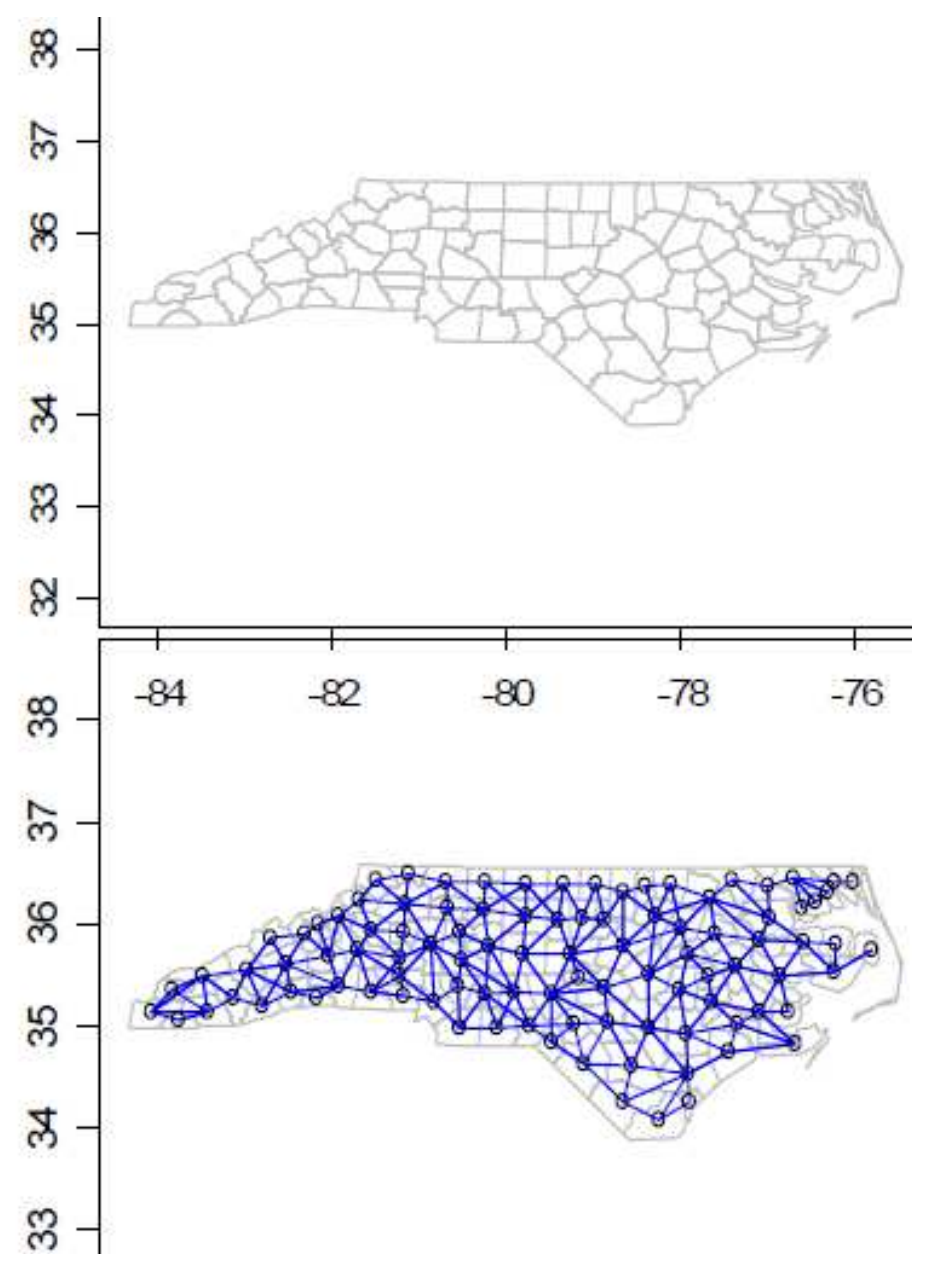




\section{Ex. de système de voisinage}

à gauche : cantons avec frontière commune

à droite : centres sont à moins de 30 miles

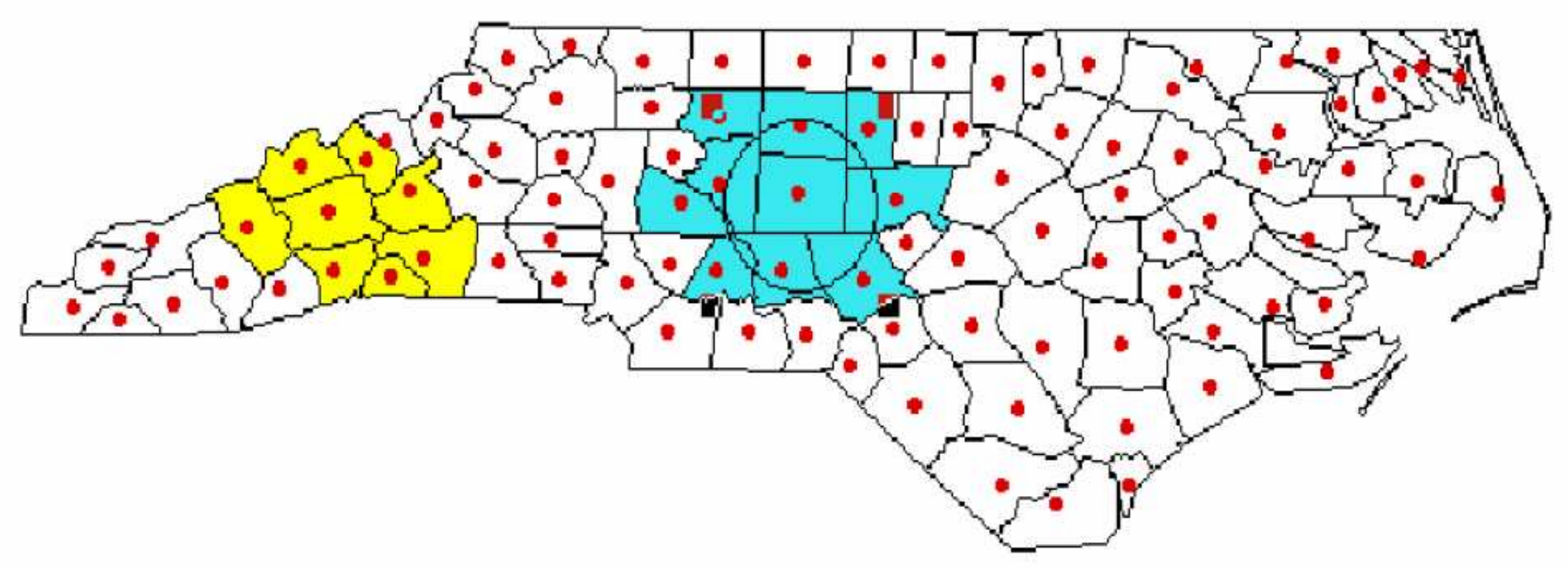


Données latticielles : « la mort subite du nourrisson »

Nombre de cas dans 100 comtés de Caroline du nord entre 19741978 (données sids de $\boldsymbol{R}$; Cressie,1993)

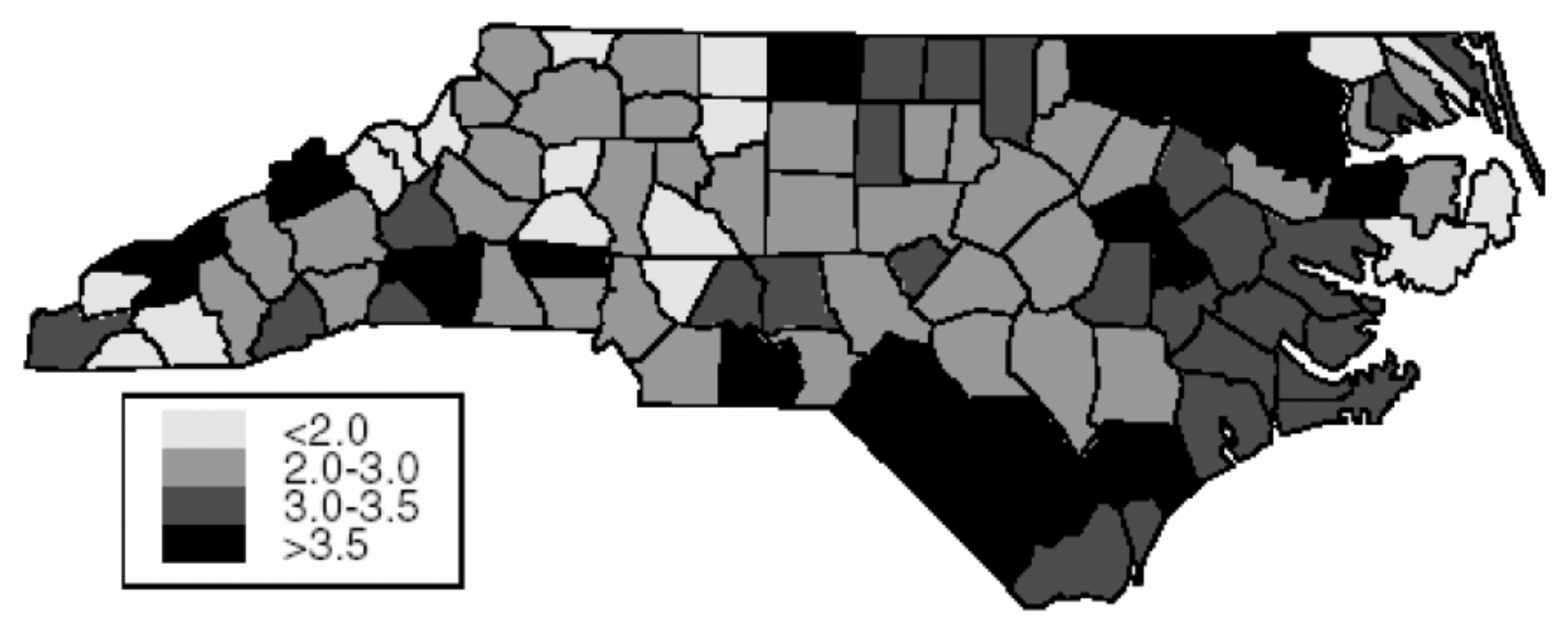




\section{nc.sids dans le package spdep}

- Voir le descriptif de nc.sids

- Représentation des 100 comtés et des 21 variables

- Graphes de voisinage 


\section{Ex. 2 : Taux groupe sanguin A}

36 contés de l'Irlande (données eire)

Percentage with blood group A in Ere

- $\mathrm{X}(\mathrm{s})=\%$ du groupe $\mathrm{A}$ dans le comté s

Covariables :

- Taux d'urbanisation (towns);

- Anciennement sous contrôle anglais (pale);

- Et d'autres ,....

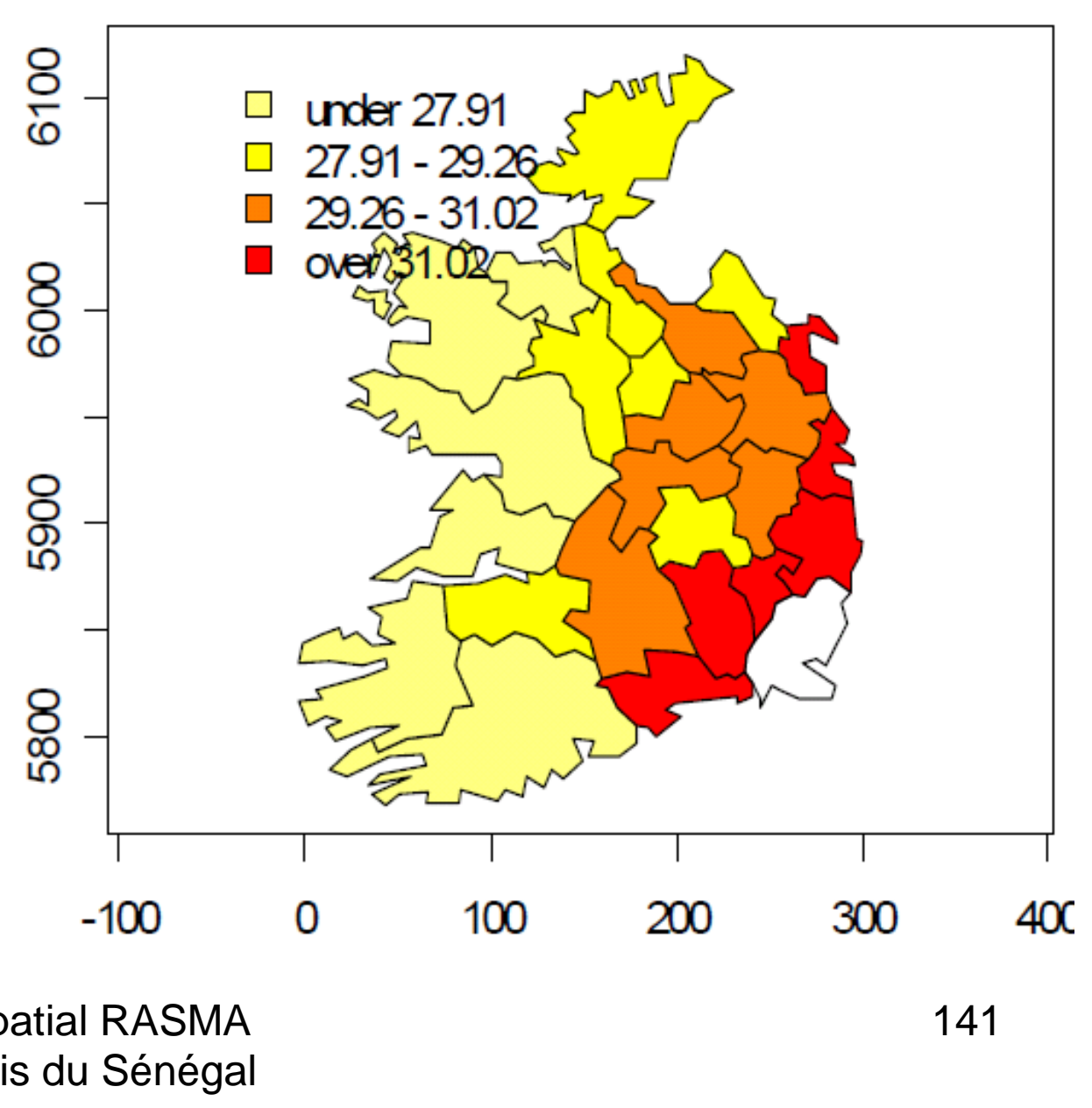




\section{Graphe de voisinage, données eire}

« avoir de la frontière commune »

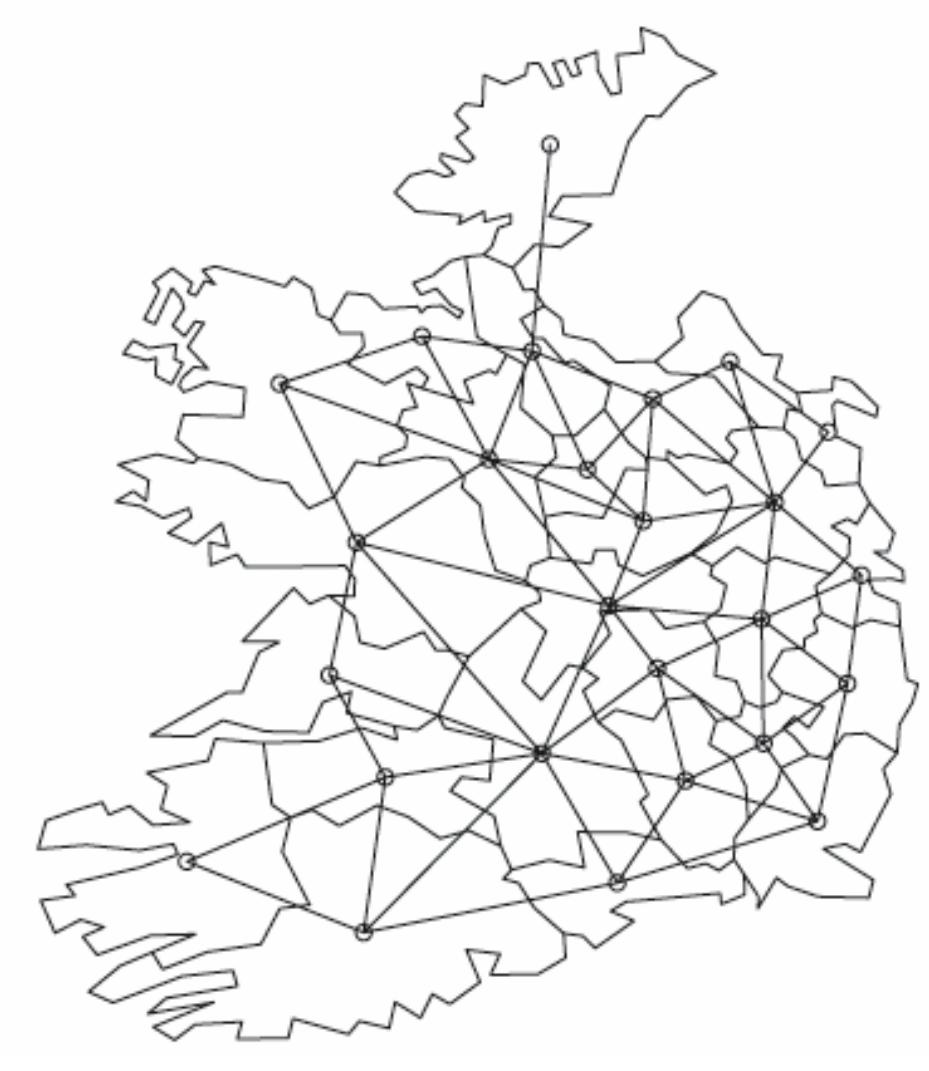




\section{Réseau régulier (agronomie, télédétection) ou non}

Choix du graphe de voisinage reste à faire Exemples de graphes réguliers symétriques:

(a) Lattice carré : voisinage aux 4 plus proches voisins (p.p.v.)

(b) Lattice carré : voisinage aux 8 - ppv

(c) Lattice triangulaire : voisinage aux 6 - ppv

(a)

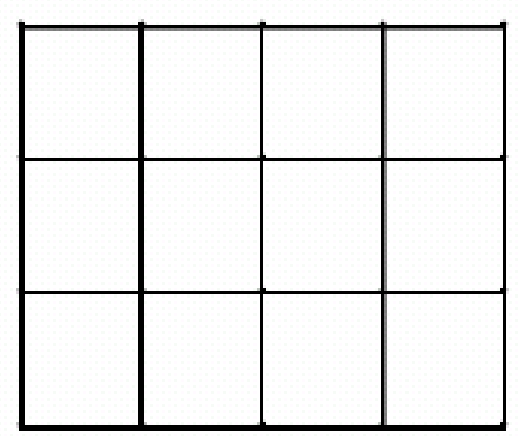

(b)

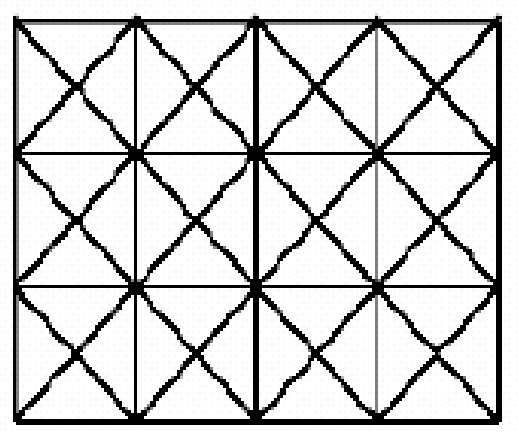

(c)

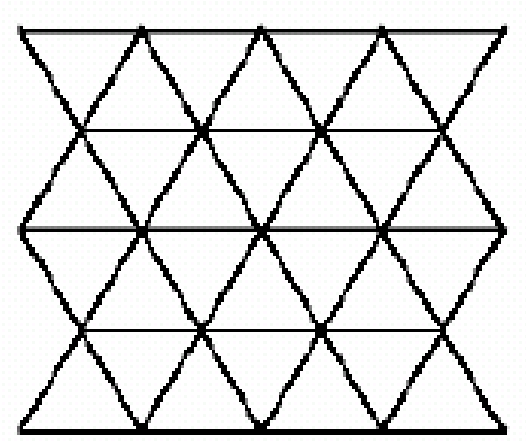




\section{$S A R$ général sur $S=\{1,2, \ldots, n\}$}

- recentrage de $X$

- Graphe et poids d'influence W + Bruit

- $n$ équations simultanées de paramètres $A$ avec $\varepsilon B B$

- X existe si $A$ inversible

- Covariance $\Sigma$ en termes des paramètres $A$

$$
\begin{aligned}
X_{t}-\mu_{t} & =\sum_{s \in S: s \neq t} a_{t, s}\left(X_{s}-\mu_{s}\right)+\varepsilon_{t} \text { ou } \\
A(X-\mu) & =\varepsilon, \text { définie si } A^{-1} \text { existe. } \\
\Sigma^{-1} & =\sigma_{\varepsilon}^{-2}\left\{{ }^{t} A A\right\}
\end{aligned}
$$


SAR général : graphe et poids

$S=$ ensemble des sites

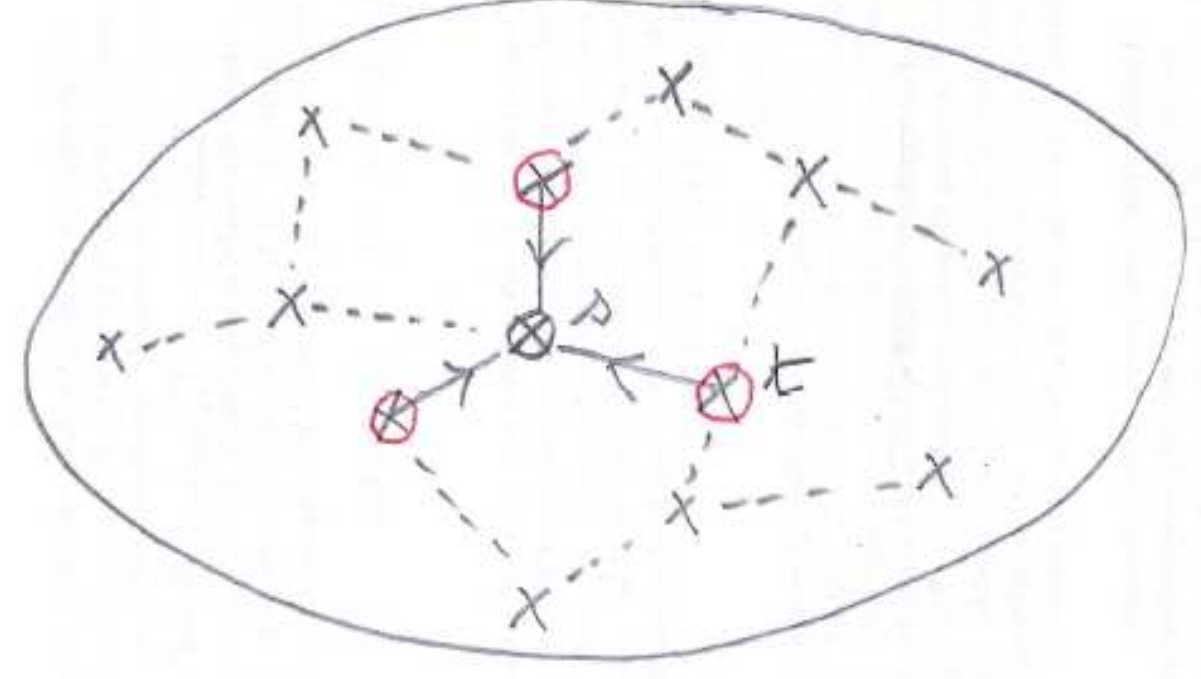

Modèle SAR

$A=$ matrice

d'inference sur tout $S$

$\otimes$ influence $\otimes=s, \quad a(s, t)=$ poids influence $t \rightarrow s$

Atelier Spatial RASMA

145

Saint Louis du Sénégal 


\section{Spécifier une SAR}

- $\partial s=$ voisins de $s$ (symétrique ou non)

- Dépendance « locale »: $X(s)=F(X(\partial s), \theta)+\varepsilon(s)$

- F linéaire via $\theta$ inconnu et $W$ matrice de poids connus $W=\{W(t, s), t$ voisins de $s\}$

- $\varepsilon$ un BB (éventuellement gaussien)

Exemple : $\rho=$ corrélation spatiale, $(I-\rho W)$ inversible

$$
X_{t}=\rho \sum_{s: s \neq t} w_{t, s} X_{s}+\varepsilon_{t}, \text { ou } X=\rho W X+\varepsilon
$$


Choix de la matrice de poids de voisinage W

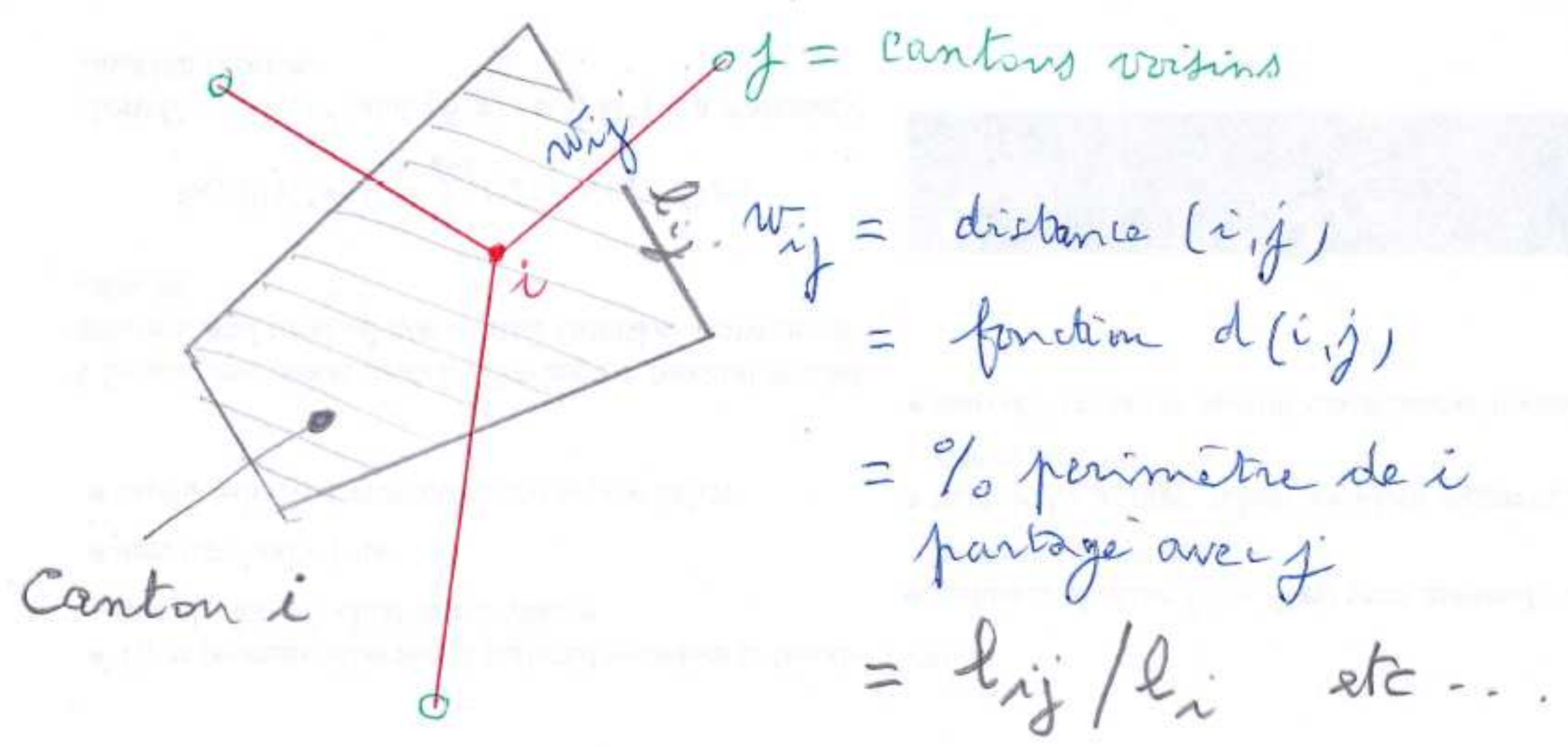

Atelier Spatial RASMA

147

Saint Louis du Sénégal 


\section{Choix ad hoc de W}

- Fonction des distances inter-centres, des (portions) de frontières communes, des réseaux de communications entre deux cellules, etc...

- Paramètres $y$ et $t$ préalablement calibrés

$$
\begin{gathered}
\cdot w_{i j}=\left\|\mathbf{s}_{i}-\mathbf{s}_{j}\right\|^{-\gamma}, \gamma \geq 0 \\
\cdot w_{i j}=\exp \left\{\left\|\mathbf{s}_{i}-\mathbf{s}_{j}\right\|^{-\gamma}\right\} \\
\cdot w_{i j}=\left(l_{i j} / l_{i}\right)^{\gamma} \text { where } l_{i j} \text { is the length } \\
\text { perimeter of the border of area } i
\end{gathered}
$$

$$
\text { - } w_{i j}=\left(l_{i j} / l_{i}\right)^{\tau} /\left\|\mathbf{s}_{i}-\mathbf{s}_{j}\right\|^{-\gamma} \text {. }
$$




\section{SAR stationnaire sur $Z^{* * 2}$}

- bruit blanc : $\eta_{t}$

(gaussien ou non)

- Équations avec variables «spatialement retardées»

$$
X_{t}=\sum_{s \in R} a_{s} X_{t-s}+\eta_{t} .
$$

- $X$ existe si $P \neq 0$ sur le tore :

$$
P\left(e^{i \lambda}\right)=1-\sum_{s \in R} a_{s} e^{i^{t} \lambda s} .
$$

- Graphe $R$ de voisinage orienté (ou non) 
Exemples de SAR

Semi causal Espace $\times$ Tempis

$$
X_{s t}=\alpha X_{s, t-1}+\beta\left(X_{s-1, t}+X_{s+1, t}\right)+\eta_{s t}
$$

Isothopique anx $4 \mathrm{kpr}$

$$
x_{\text {st }}=\alpha(\text { somme } 4 \mathrm{ppv})+\eta \text { st }
$$

Factorisont (cousal) aux 3-ppV

$$
X_{s t}=\alpha X_{s-1, t}+\beta X_{s, t-1}-\alpha \beta X_{s-1, t-1}+\eta_{s t}
$$
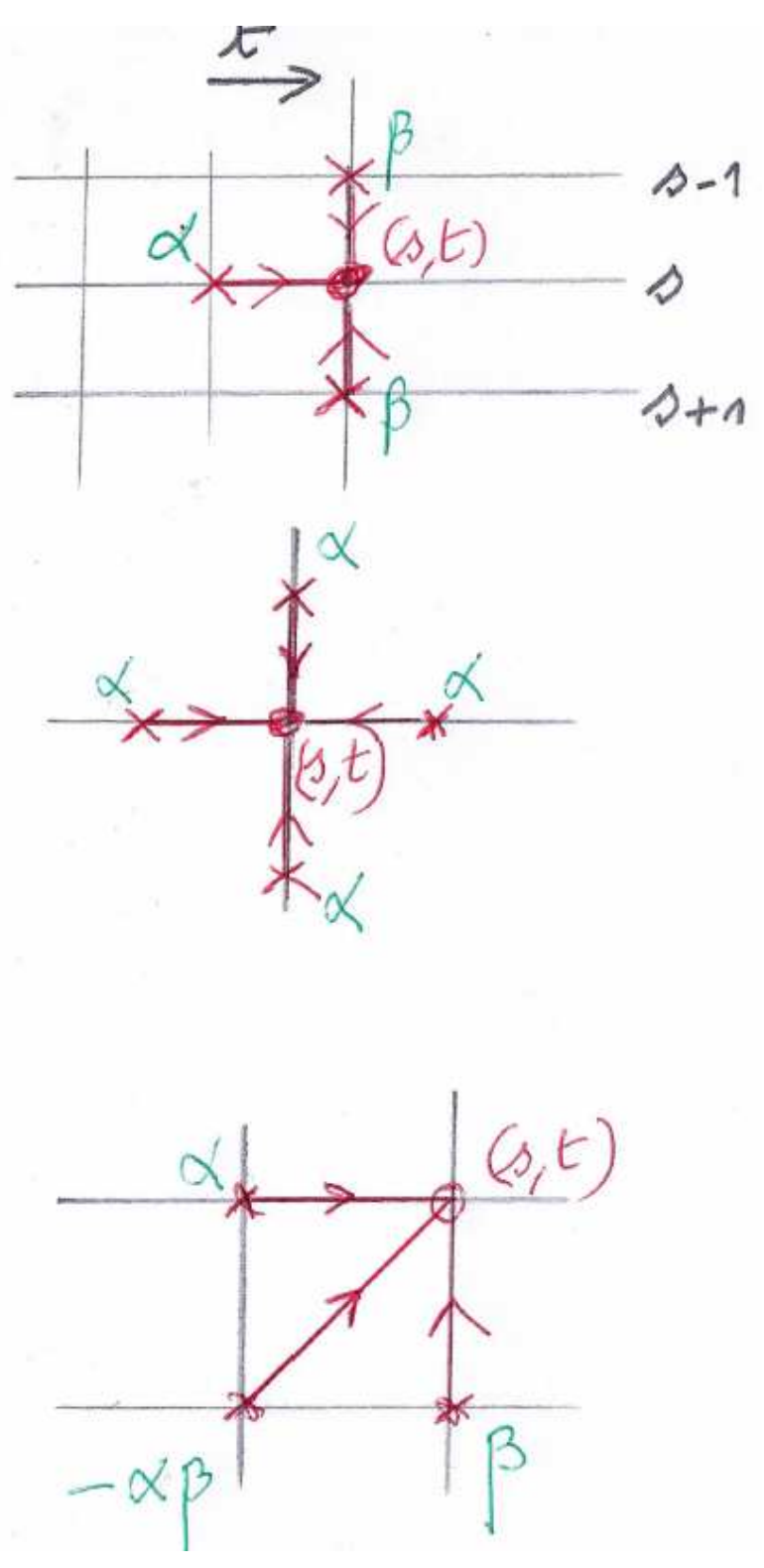

Saint Louis du Sénégal 


\section{Précautions sur un SAR}

- Un SAR bilatéral $\neq>A R$ causal (pour l'ordre lexicographique; cf. exemple dans le polycopié)

- Sans contrainte, un SAR non identifiable

- L'estimation des MCO est non convergente

- Avantage : SAR est parcimonieux en paramètres 


\section{$A R$ conditionnel général $(C A R)$ sur $S$}

- Écrire l'espérance conditionnelle de $X(t)$ sur autres $X$ :

Les résidus e sont corrélés entre euX, décorrélés des $X$ :

$$
\begin{gathered}
X_{t}=\sum_{s \in S: s \neq t} c_{t, s} X_{s}+e_{t}, \quad \forall t \in S \\
\operatorname{Var}\left(e_{t}\right)=\sigma_{t}^{2}>0, \operatorname{Cov}\left(X_{t}, e_{s}\right)=0 \text { si } t \neq s .
\end{gathered}
$$

- Notations : $D$ diagonale des résidus, $\Sigma=\operatorname{Cov}(X)$

$C$ paramètres CAR. On a l'identité :

$$
\Sigma^{-1}=D^{-1}(I-C)
$$

$\rightarrow$ contraintes sur les paramètres du CAR 


\section{Contraintes sur les paramètres d'un $C A R$}

$$
\begin{gathered}
X_{t}=\sum_{s \in S: s \neq t} c_{t, s} X_{s}+e_{t}, \\
(I-C) \Sigma=D . \\
c_{t, s} \sigma_{s}^{2}=c_{s, t} \sigma_{t}^{2}, \quad \forall t \neq s \in S .
\end{gathered}
$$




\section{AR Conditionnelle (CAR) stationnaire}

$$
\begin{aligned}
& X_{t}=\sum_{s \in L} c_{s} X_{t-s}+e_{t} \text { avec, si } s \in L^{+}: c_{s}=c_{-s} \\
& \forall s \neq t: \operatorname{Cov}\left(e_{t}, X_{s}\right)=0 \text { et } E\left(e_{t}\right)=0
\end{aligned}
$$

- L'espérance conditionnelle linéaire de $X(s)$ sur les autres $X(t)$

- $e(t)$ est décorrélé des $X(s)$ pour $s \neq t$

- Le graphe d'un CAR symétrique ainsi que les $c$.

- Le résidu conditionnel e est un bruit coloré (c-à-d corrélé) :

$$
\operatorname{Cov}\left(e_{t}, e_{t+s}\right)=\left\{\begin{array}{c}
\sigma_{e}^{2} \text { si } s=0, \\
-\sigma_{e}^{2} c_{s} \text { si } s \in L
\end{array} \text { et } \operatorname{Cov}\left(e_{t}, e_{t+s}\right)=0\right. \text { sinon }
$$


$C A R$ : espérance conditionnelle linéaire et résidu

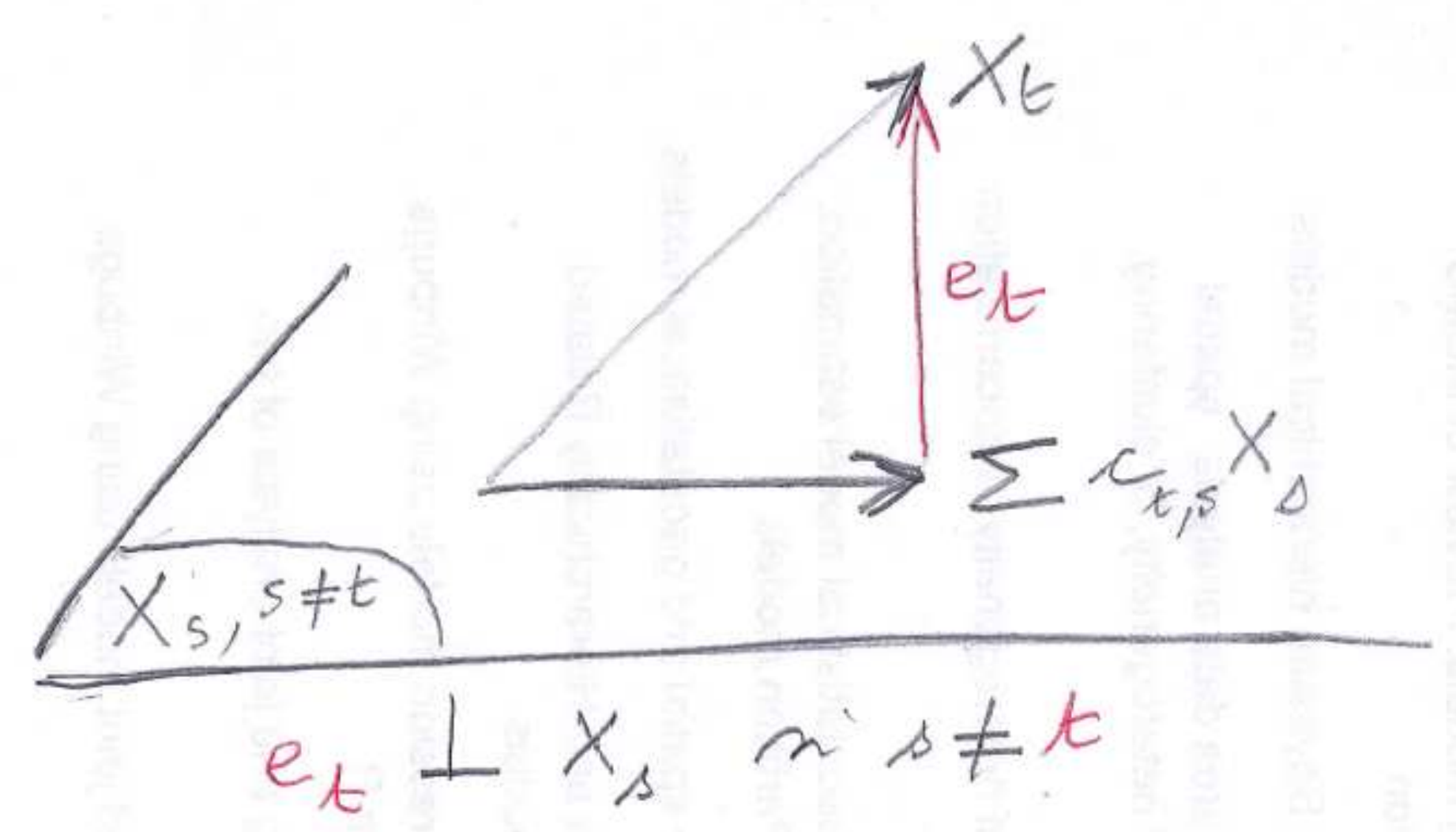

Atelier Spatial RASMA

Saint Louis du Sénégal

155 


\section{SAR ou CAR : pour résumer}

- Un SAR est spécifié par $n$ équations simultanées à résidus $B B$

- Un CAR est spécifié par ses « espérances conditionnelles linéaires ». Le bruit résiduel est coloré 


\section{CAR ou SAR ?}

- Tout SAR est un CAR

- Si S fini, CAR $\equiv$ SAR ( $\neq$ sinon)

- Écriture CAR intrinsèque, celle d'un SAR non

- Estimation MCO

$$
\begin{aligned}
& \text { d'un CAR convergente } \\
& \text { d'un SAR non }
\end{aligned}
$$

- SAR : plus parcimonieux en nombre de paramètres

- CAR : contraintes sur les paramètres 


\section{Correspondances des graphes : $R$ d'un SAR $\leftarrow$ et $\rightarrow$ G d'un CAR}

- $\boldsymbol{R}$ graphe du SAR : orienté

- G du CAR : non orienté, le « double » de $\boldsymbol{R}$ :

Le graphe $\mathcal{G}$ de la représentation markovienne $C A R$ de $X$ est

$$
\langle t, s\rangle_{\mathcal{G}} \Longleftrightarrow\left\{\begin{array}{l}
\text { soit }\langle t, s\rangle_{\mathcal{R}}, \\
\text { soit }\langle s, t\rangle_{\mathcal{R}} \\
\text { soit } \exists l \in S \text { t.q. }\langle l, t\rangle_{\mathcal{R}} \text { et }\langle l, s\rangle_{\mathcal{R}}
\end{array}\right.
$$




\section{Exemple de correspondance}

(a) SAR et $R$

(b) CAR associé et $G$

en pointillé : voisinage CAR de •

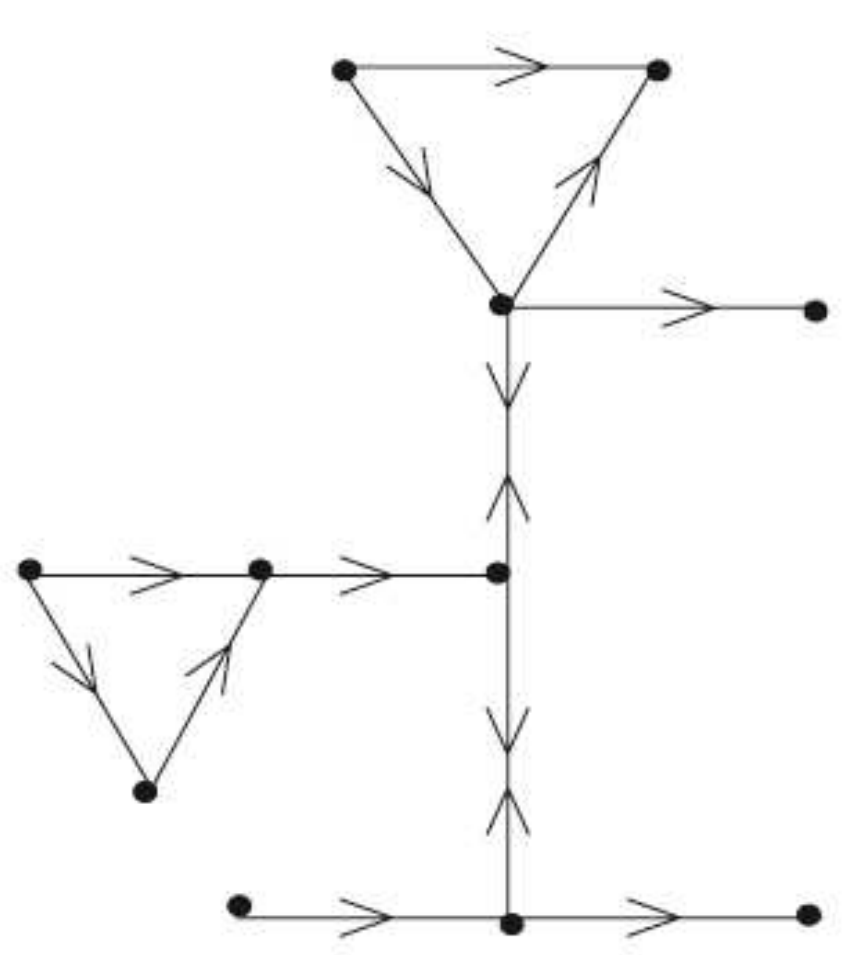

(a)

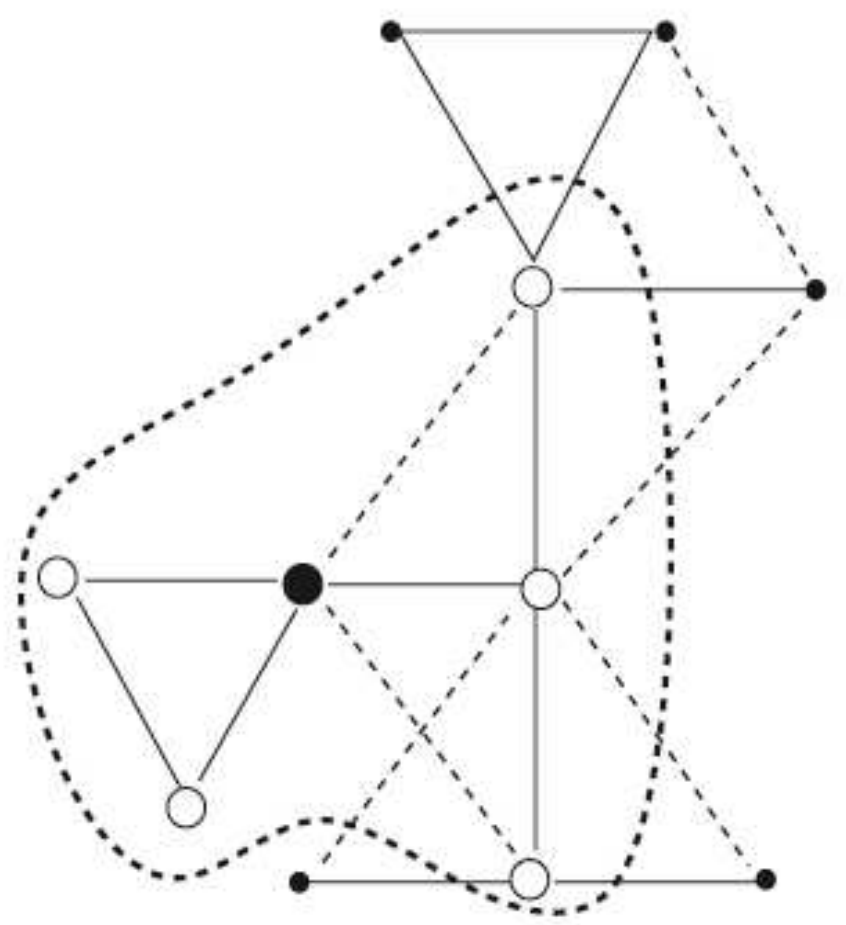

(b) 


\section{SAR aux 4 ppv et $C A R$ associé}

- $S A R$ aux 4-ppv avec $R=\{(1,0),(-1,0),(0,1),(0,-1)\}$

$X_{s, t}=a\left(X_{s-1, t}+X_{s+1, t}\right)+b\left(X_{s, t-1}+X_{s, t+1}\right)+\varepsilon_{s, t}$

$\rightarrow C A R$ aux 12-ppv (cf. poly. pour les coeff. $c(s)$ ) avec

$$
L_{+}=\{(1,0),(2,0),(1,1),(0,1),(0,2),(1,-1)\}
$$

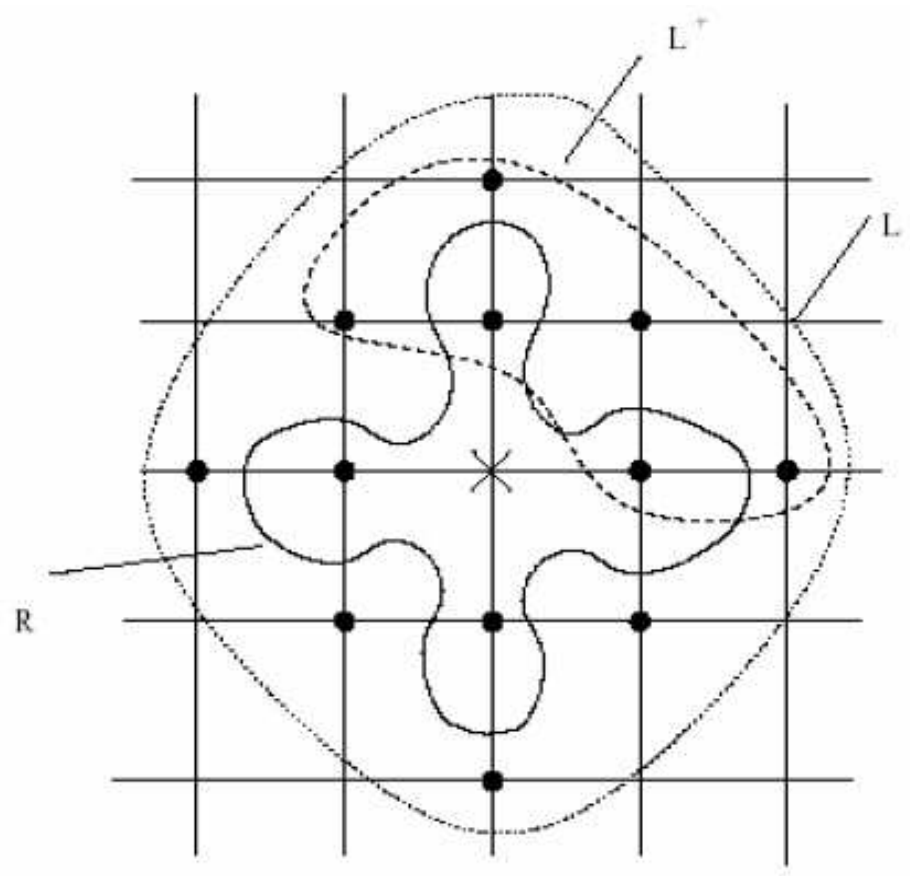

avec un gain de prédiction

$$
k^{* * 2}={ }^{\left(1+2 a^{2}+2 b^{2}\right)^{-1}}
$$




\section{SAR factorisant aux $3-\mathrm{ppv}$ et CAR aux 8-ppv associé}

$R=$ support du $S A R$ et $L=$ support du CAR (8 voisins) $X_{s, t}=\alpha X_{s-1, t}+\beta X_{s, t-1}-\alpha \beta X_{s-1, t-1}+\varepsilon_{s, t}$

$\kappa^{2}=\left(1+\alpha^{2}\right)^{-1}\left(1+\beta^{2}\right)^{-1}$

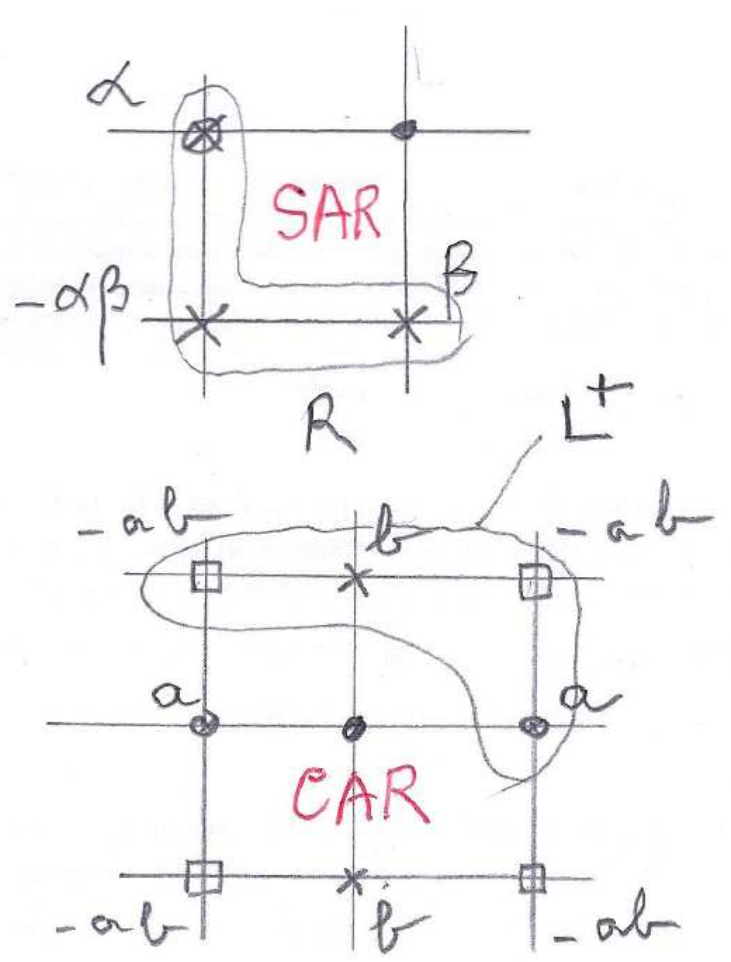

$$
\begin{aligned}
& a=\frac{\alpha}{1+\alpha^{2}} \\
& b=\frac{\beta}{1+\beta^{2}}
\end{aligned}
$$




\section{SARX avec exogènes}

- $\quad X$ endogène, $Z$ matrice des exogènes

- Matrice de retard $W$ sur endogène et exogène

- 3 types de variables expliquent $X(t)$ :

(1) endogène retardée $W X$,

(2) exogène $Z$ et

(3) exogène retardée WZ.

- On obtient facilement $E(X)$ et $\operatorname{Cov}(X)$

$$
X=\rho W X+Z \beta+W Z \gamma+\varepsilon, \rho \in \mathbb{R}, \beta \text { et } \gamma \in \mathbb{R}^{p}
$$




\section{Deux modèles avec exogènes}

- Modèle de Durbin spatial $(X-Z \beta \sim S A R(\rho, W))$

$$
(I-\rho W) X=(I-\rho W) Z \beta+\varepsilon
$$

- Modèle à décalage spatial $(\gamma=0)$

$$
X=\rho W X+Z \beta+\varepsilon
$$




\section{Auto - corrélation de Moran}

- $X$ sur $S=\{1,2, \ldots, n\}$ centré (adaptation si modèle de régression sur $E(X))$

- $W(i, j)$ matrice de poids $i \rightarrow j, i \neq j(W(i, i)=0)$ donnée

- W-auto-corrélation de Moran :

$$
\begin{aligned}
& : \quad I_{M}=\frac{n \sum_{i, j} w_{i, j}\left(X_{i}-\bar{X}\right)\left(X_{j}-\bar{X}\right)}{s_{0} \times \sum_{i}\left(X_{i}-\bar{X}\right)^{2}} \\
& s_{0}=\sum_{i, j} w_{i, j}=\text { somme des poids } W
\end{aligned}
$$




\section{Test de non corrélation spatiale $(H(0))$}

- I(M) petit de variance identifiée

$$
\begin{aligned}
\text { Sous }\left(H_{0}\right) & : E\left(I_{M}\right)=o(1) \text { et } \operatorname{Var}\left(I_{M}\right) \simeq \frac{s_{1}}{s_{0}^{2}} \\
\text { où } s_{1} & =\sum_{i, j}\left(w_{i, j}^{2}+w_{i, j} w_{j, i}\right) .
\end{aligned}
$$

- Si X gaussien, plus précisions sur E() et $\operatorname{Var}()$.

- En général, sous $(H O)$, normalité : $\frac{s_{0 n}}{\sqrt{s_{1 n}}} I_{n}^{M} \stackrel{l o i}{\longrightarrow} \mathcal{N}(0,1)$. 


\section{Indice de Geary}

Mesure la dépendance spatiale comme le fait un variogramme :

I(G) est petit si les valeurs voisines sont proches

$$
I_{n}^{G}=\frac{(n-1) \sum_{i, j \in D_{n}} w_{i j}\left(X_{i}-X_{j}\right)^{2}}{2 s_{0 n} \sum_{i \in D_{n}}\left(X_{i}-\bar{X}\right)^{2}} .
$$




\section{Loi permutationnelle d'une statistique $I(X)$}

- $X=\{X(i), i=1, n\}$ et $I(X)$ une statistique réelle

- Distribution empirique des $\{I(X(\sigma)), \sigma$ permutation $\}$

- Intervalle de confiance associé à la statistique d'ordre

- Mais $n$ ! est trop grand $\rightarrow$ le faire pour $m$ permutations choisies au hasard

$$
\mathcal{I}=\left[I_{(\alpha \sigma)}, I_{((1-\alpha) \sigma}\right]
$$




\section{Application : test de permutation de $(H O)$ : indépendance des $\{X(i), i=1, n\}$}

- Choix de I(X), l'indice de Moran : sous (HO),

$$
\left(X_{i}, i=1, \ldots, n\right) \sim\left(X_{\sigma(i)}, i=1, \ldots, n\right) .
$$

- Calcul des $I(\sigma, X)$ pour $m$ permutations au hasard (i.e. $m=1000$ ) et de l'intervalle de confiance empirique $I C(1-\alpha)$

- Calcul de I(x) pour l'observation $x$

- Si $I(x)$ n'est pas dans $I C(1-\alpha)$, rejet de $(H O)$

- Avantage : non - asymptotique, libre du modèle sur $X$

- Inconvénient : le niveau est approximatif 


\section{Données eire: groupe sanguin A}

$1-G=$ graphe de voisinage de contiguité des 26 contés

$2-w(i, j)=1 /(\mathrm{nb}$ voisins de i) si $j$ est voisin de $i$
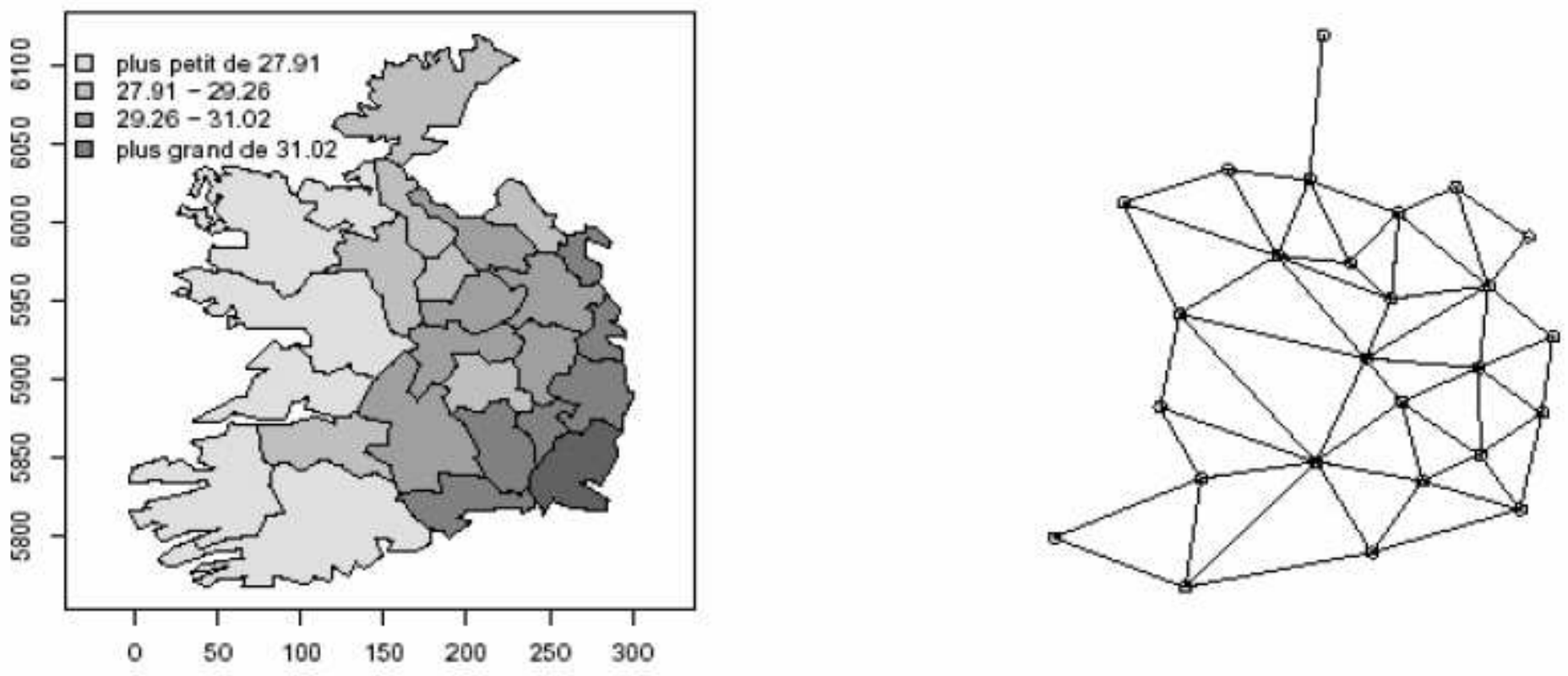


\section{Indice de Moran (Geary) + indices réduits $t(a)$ probabilités $p(a)$ de dépassement}

$1-I=$ index, $t$ (a) index réduit, asymptotique gaussienne $2-p(a)=$ proba de dépassement de $t(a)$

3 - pmc(a) pour le test de permutation MC $(m=1000$ permutations)

\begin{tabular}{|l|c|c|c|c|}
\hline & Index & $t^{a}$ & $p^{a}$ & $p_{M C}^{a}$ \\
\hline Moran & 0.554 & 4.663 & 0 & 0.001 \\
Geary & 0.380 & -4.547 & 0 & 0.001 \\
\hline
\end{tabular}




\section{Le package spdep: étude sur l'exemple des données eire}

- Données i.e. : eire groupe sanguin en Irlande

- Tester bloc par bloc l'exemple de traitement de ces données

1 - représentation des données (valeur, graphe de voisinage)

2 - indice de Moran et test de non corrélation spatiale

3 - régression sur town et pale, analyse des résidus (indice de Moran, SAR sur les résidus)

- Autres données sur la consommation intérieure 


\section{Quelques programmes de spdep}

- moran, moran.test et moran.mc

- lagsarlm : estimation du MV d'un SAR avec covariables

$$
y=\text { rho } W y+X \text { beta }+e
$$

- knearneigh : matrice des k-ppv pour un choix de distance

- Im.morantest : test de Moran pour l'auto-corrélation spatiale des résidus d'un modèle linéaire

- lm.morantest.exact : test exact de non corrélation

- sp.correlogram : corrélogramme spatial pour l'indice de moran et d'autres programmes .... 


\section{Estimation d'une régression spatiale}

\section{$X=Z \delta+\varepsilon$ où $\operatorname{Cov}(\varepsilon)=\Sigma$}

- Estimation MCO de $\delta$ :

$$
\widetilde{\delta}=\left({ }^{t} Z Z\right)^{-1{ }^{t}} Z X .
$$

- Sous bonnes conditions, consistance des MCO

$\rightarrow$ MCO bonne estimation initiale dans une procédure itérative type MCQG 


\section{Moindres Carrés Généralisés (MCG)}

- Si $\Sigma=\operatorname{cov}(\varepsilon)$ est connue, le BLUE vaut

$$
\begin{gathered}
\hat{\delta}_{M C G}=\left({ }^{t} Z \Sigma^{-1} Z\right)^{-1 t} Z \Sigma^{-1} X ; \\
\operatorname{Var}\left(\hat{\delta}_{M C G}\right)=\left({ }^{t} Z \Sigma^{-1} Z\right)^{-1} .
\end{gathered}
$$

- Si $X$ gaussien, c'est l'EMV, efficace

- En général $\Sigma$ inconnue $\rightarrow$ MC Quasi G (MCQG) 


\section{MCQG : $\Sigma=\Sigma(\theta), \theta$ inconnu}

1. estimer $\delta$ par MCO : $\tilde{\delta}=\left({ }^{t} Z Z\right)^{-1 t} Z X$.

2. calculer les résidus des $M C O: \tilde{\varepsilon}=X-Z \tilde{\delta}$

3. $\operatorname{sur}$ la base de $\tilde{\varepsilon}$, estimer $\tilde{\theta}$ (pour $\Sigma(\theta)$ ou $2 \gamma(\theta)$ ) par MC.

4. estimer $\Sigma$ par $\tilde{\Sigma}=\Sigma(\tilde{\theta})$ puis $\delta$ par $M C G(\tilde{\Sigma})$. Itérer. 


\section{Régression Gaussienne : MV}

- Régression à covariance non sphérique :

$$
X \sim \mathcal{N}_{n}(Z \delta, \Sigma(\theta))
$$

- Mardia-Marshall donnent le comportement limite de l'EMV de $(\theta, \partial)$ (cf. poly)

- Log-vraisemblance est explicite :

$2 l(\delta, \theta)=\log |\Sigma(\theta)|+{ }^{t}(X-Z \delta) \Sigma^{-1}(\theta)(X-Z \delta)$ 


\section{Données eire : 2 modèles de régression avec 2 covariables}

- towns (densité urbaine) et

- pale (binaire, 1 si colonisation anglaise, 0 sinon)

(R1) : cste, towns et pale + résidus i.i.d.

(R2) : cste, pale + résidus SAR aux ppv : $r=(\rho W) r+e$

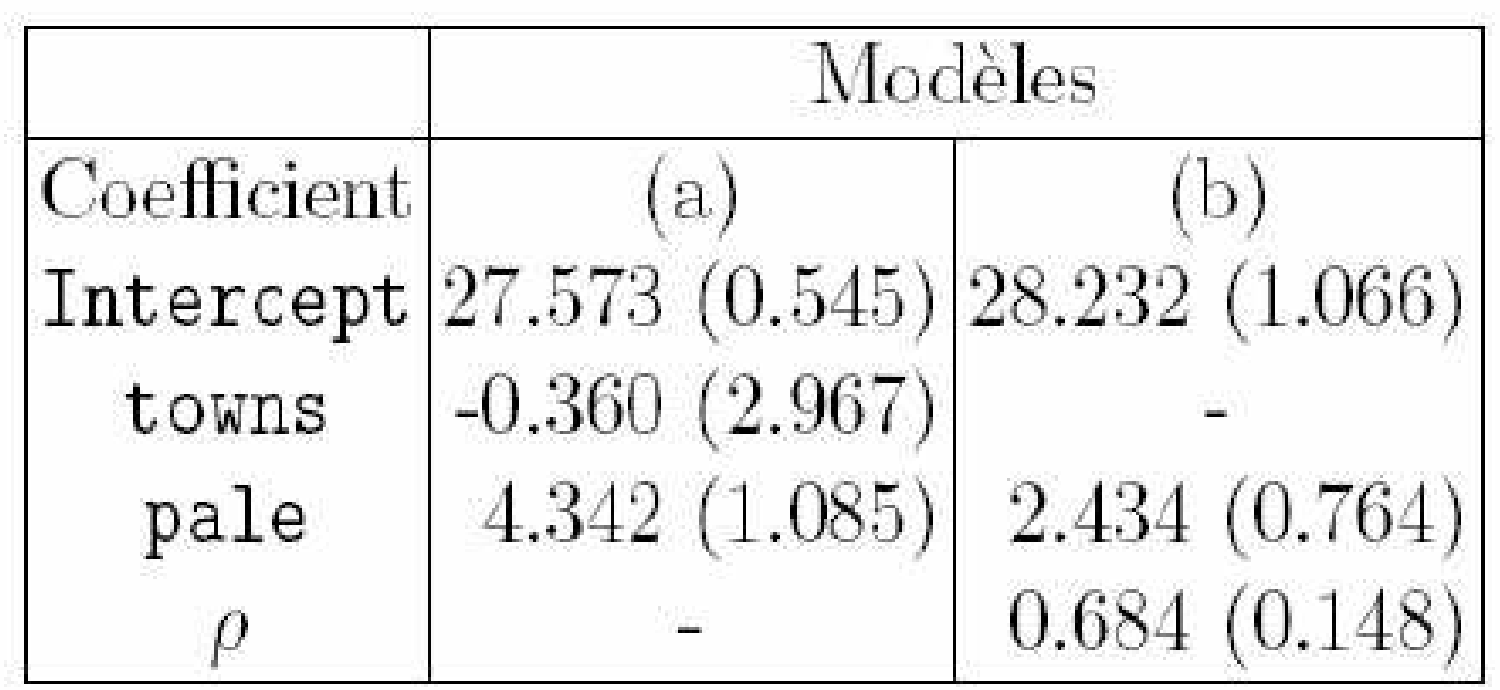




\section{Spdep, le package de $\boldsymbol{R}$ pour les SAR, SARX, Indice de Moran, test Monte Carlo, ...}

- spdep : spatial dependence, weighting schemes, statistics, models.

anova.sarlm : compare des SAR deviance.sarlm

errorsarlm : MV du modèle de

Durbin spatial, $Y=X b+u$ ou

$$
u=r W u+e
$$

Données : sids, eire, getisord

(télédétection)

moran, moran.mc test de

permutation), moran.test

(gaussien)

plot.spcor : corrélogramme spatial, etc.......
- Autres packages :

nlme : linear and non linear (mixed effect) models

$\boldsymbol{g l s}$ (et $\boldsymbol{g n} \boldsymbol{l} \boldsymbol{s})$ : GLS pour modèle linéaire (non linéaire), avec ou sans effet aléatoire.

logLik.g(n)ls : MV pour ces modèles

- RandomFields : simulation et analyse des RF. 


\section{Processus Ponctuels Spatiaux}

- Ici, c'est la répartition spatiale des points où ont lieu les observations qui est aléatoire.

- Hypothèse de base a tester : la répartition est homogène et au hasard (CSR pour Complete Spatial Randomness), celle d'un PP de Poisson.

- D’autres répartitions sont plus régulières (noyau dur), d'autres moins (agrégats). 


\section{Répartition de 97 fourmilières}

(données ants du package spatstat)

Question : la répartition s'est elle faite au hasard?

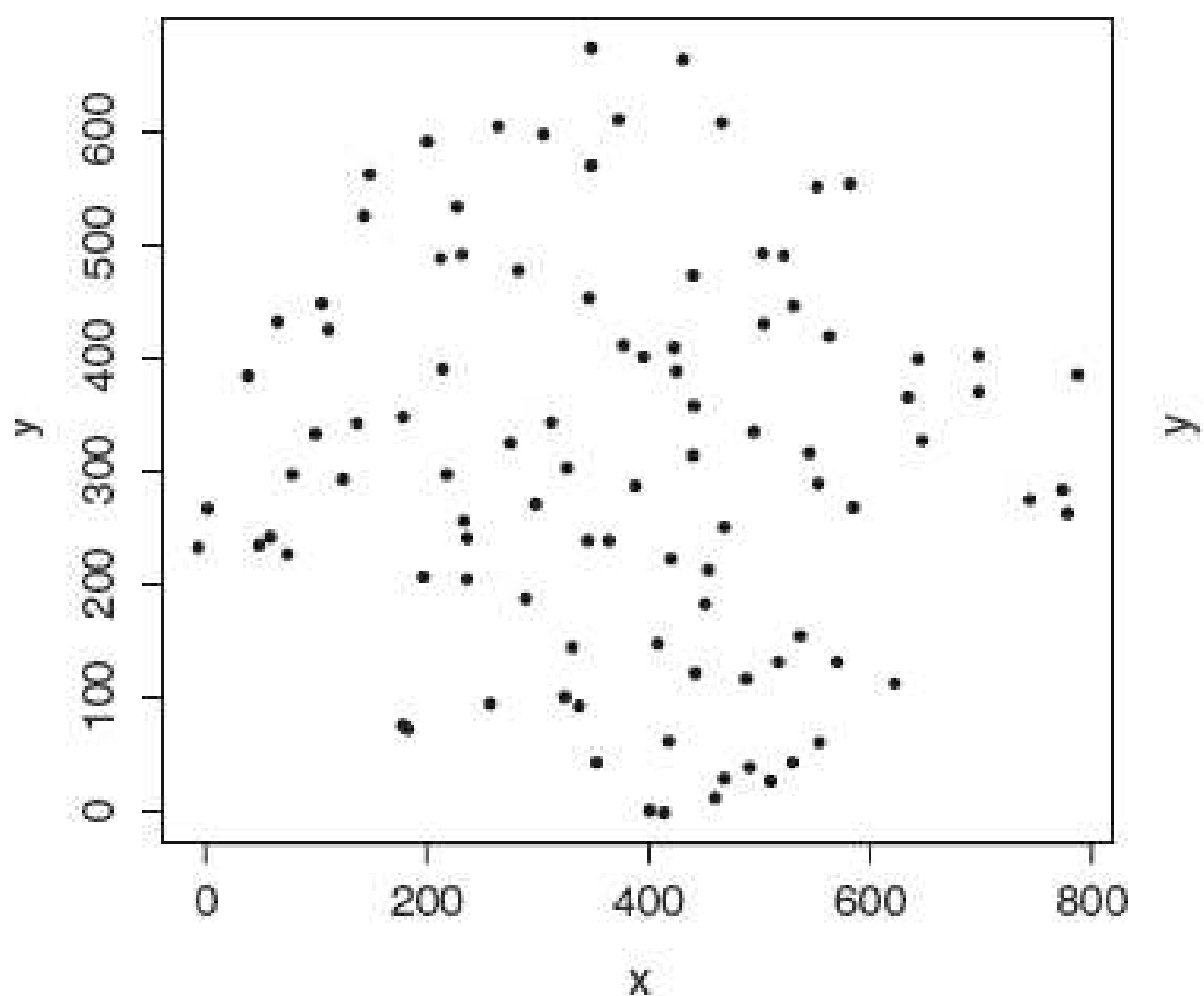

(a) 


\section{2 centres de cellules d'une coupe histologique}

(données cells de spatstat)

1 - La répartition est - elle au hasard ?

2 - Sinon (+ de régularité), quel modèle proposer ?

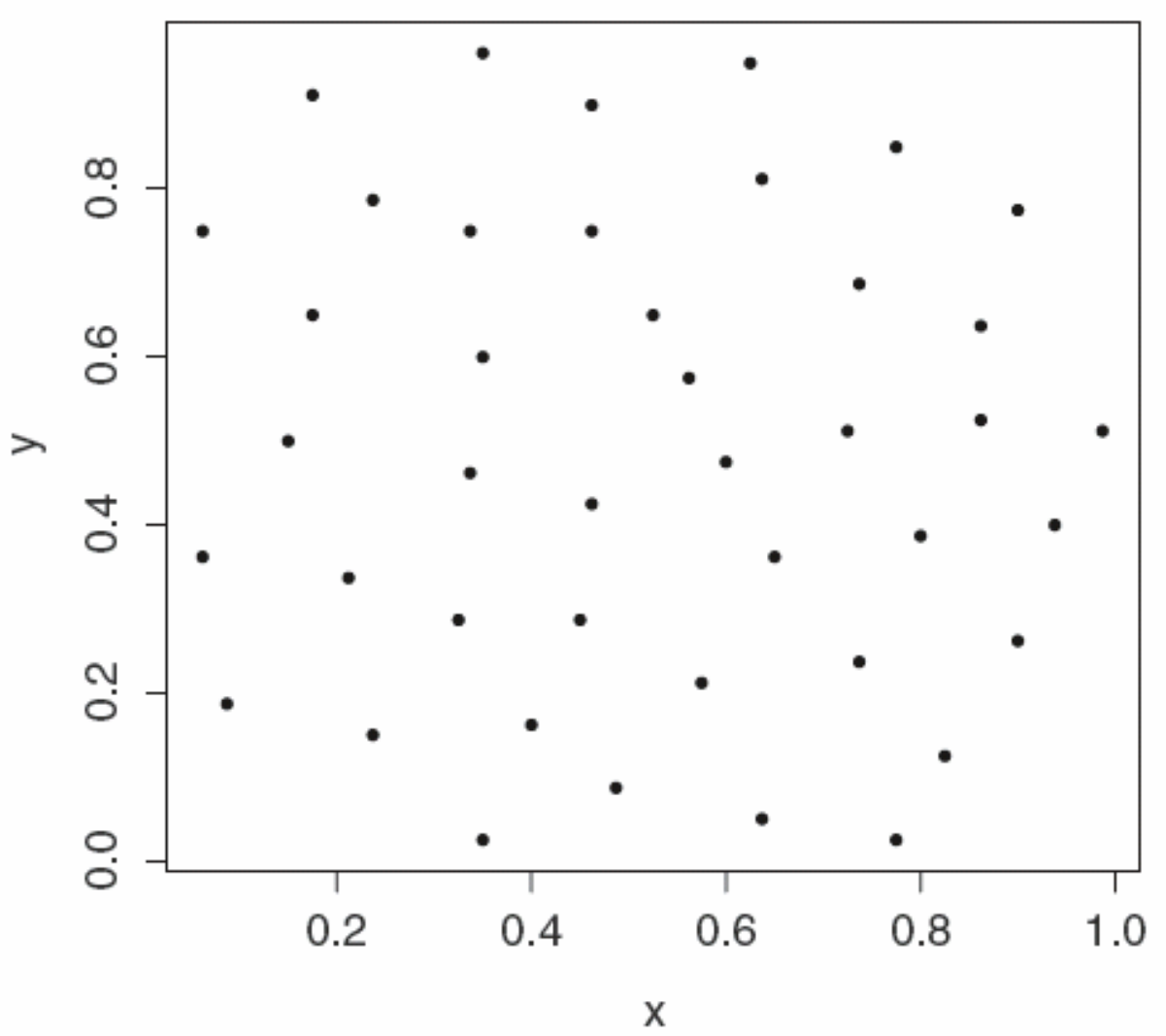

Atelier Spatial RASMA 


\section{6 pins d'une forêt finlandaise}

(données finpines de spatstat)

1 - La répartition est elle au hasard?

2 - Sinon (des aglomérats ?), quel modèle proposer?

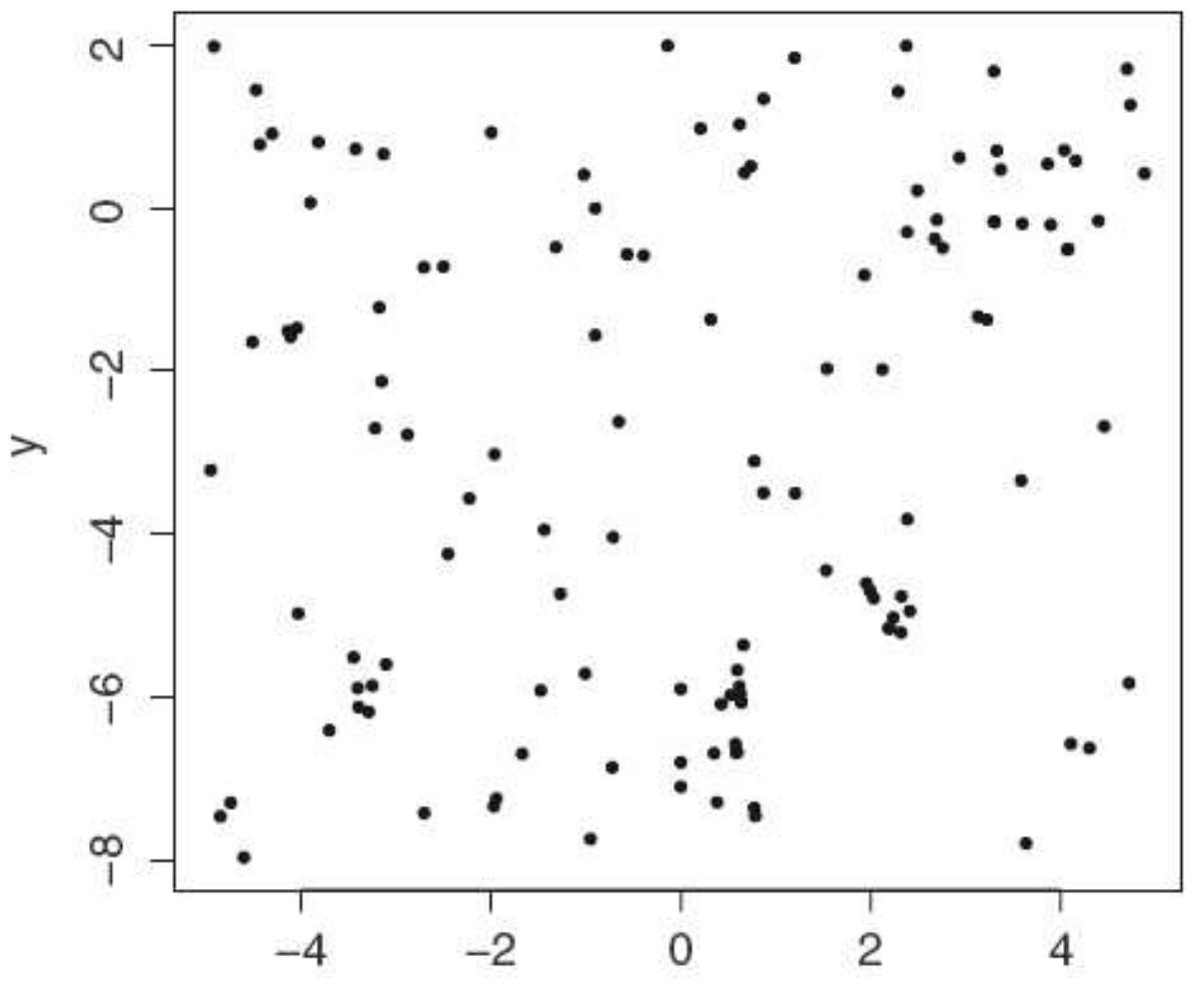

Atelier Spatial RASMA 


\section{Modèle de Processus Ponctuel X(PP)}

- Configuration $x$ : ensemble fini de points de la fenêtre d'observation $S$

- Configuration à $n$ points : $x=\{x(1), x(2), \ldots, x(n)\}$

- $E(n)=$ espace des configurations à $n$ points

- $E=U E(n)$ : l'espace exponentiel de toutes les configurations, réunion des $E(n)$

- $N(A)$ : le nombre de points de $X$ dans $A$

- Loi de $X$ : loi jointe de toutes les variables de comptage $N(A), A$ partie de $S$ 


\section{PP de Poisson homogène d'intensité $\lambda$}

$P P P(\lambda)$ : répartition spatiale homogène et au hasard

(1) $N(A)$ suit une loi de Poisson de paramètre $\lambda|A|$

(2) La répartition sur $A$ est uniforme

$(1-2) \equiv\left(1-2^{*}\right)$ où

$\left(2^{*}\right)$ : si $A$ et $B$ sont disjoints, $N(A)$ et $N(B)$ sont indépendants 


\section{Deux répartitions spatiales homogènes à 70 points}

- (a) une répartition de Poisson homogène

- (b) une répartition «à $r$ - noyau dur» homogène (couples de points à distance $<r=3.5$ sont interdits)

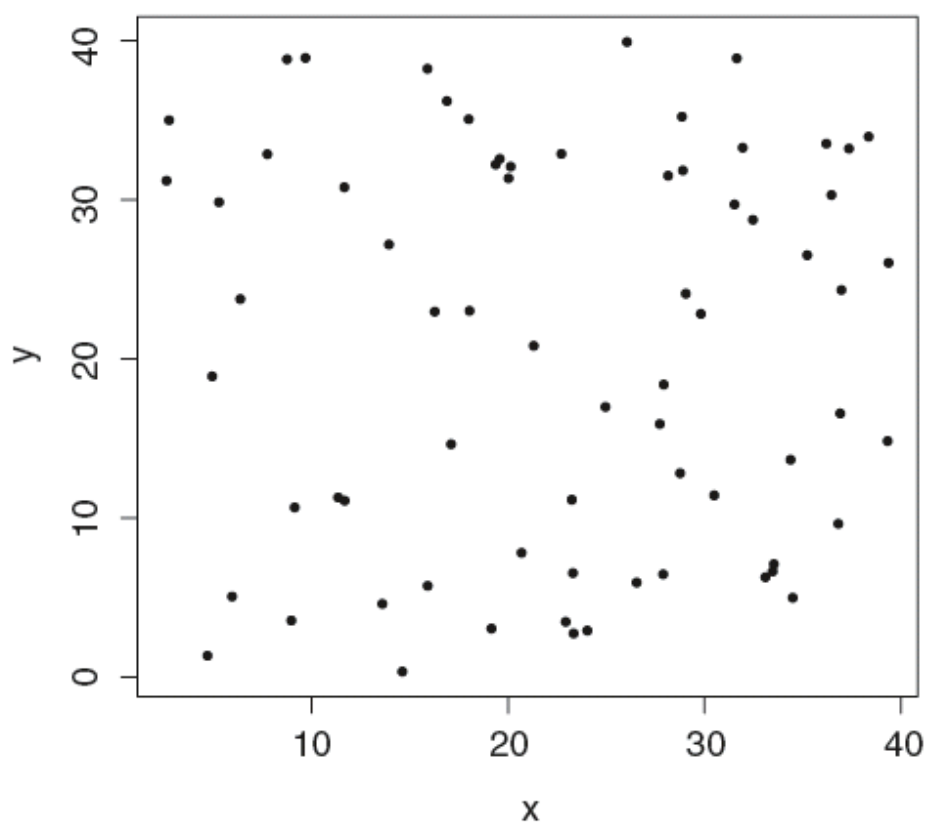

(a)

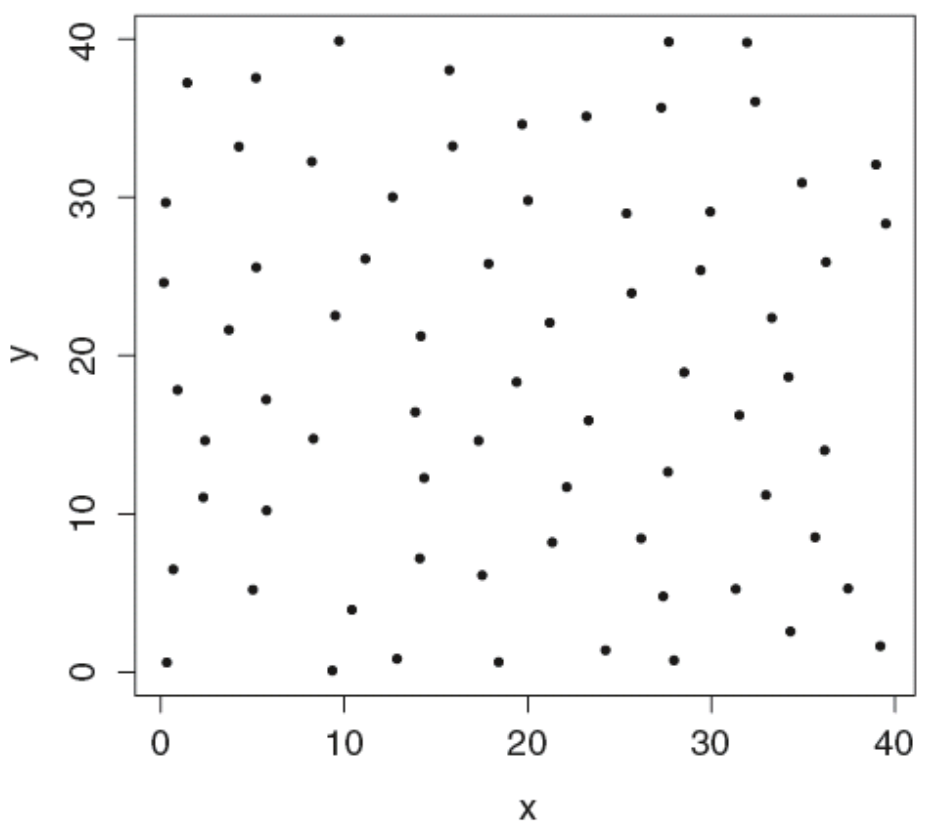

(b) 


\section{PPP inhomogène d'intensité $\lambda(\bullet)$}

Soit $\lambda(\bullet)$ une mesure sur la fenêtre d'observation $S$

$X$ est un $\operatorname{PPP}(\lambda(\bullet))$ si :

(1) $N(A)$ suit une loi de Poisson de paramètre $\lambda(A)$

(2) si $A$ et $B$ sont disjoints, $N(A)$ et $N(B)$ sont indépendants 


\section{Simulation d'un $\operatorname{PPP}(\lambda(\bullet))$}

Supposons que pour tout $x: \lambda(x) \leq c<\infty$

La méthode par effacement de points est la méthode de simulationpar rejet suivante :

1. Simuler $\boldsymbol{X}^{*}$, un PPP homogène d'intensité $c$;

2. Effacer indépendamment un $x$ (i) de $x^{*}$ avec la probabilité $p(x(i))=\{1-\lambda(x(i)) / c\}$. 


\section{Simulation de 2 PPP inhomogènes}

( $\lambda$ plus grand $\rightarrow$ fond plus clair)

Deux intensités inhomogènes

(a) : $\lambda(x, y)=400 e^{-3 x}$ et (b) $\lambda(x, y)=800 \times|0.5-x| \times|0.5-y|$

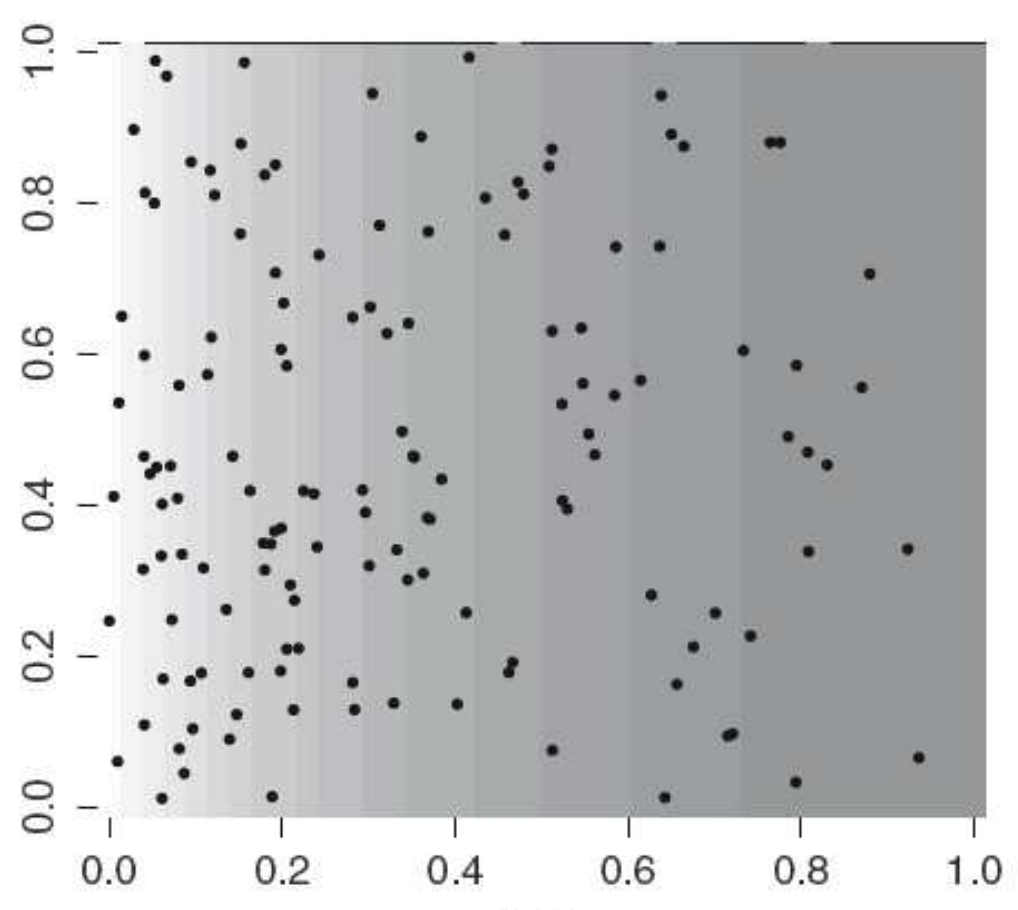

(a)

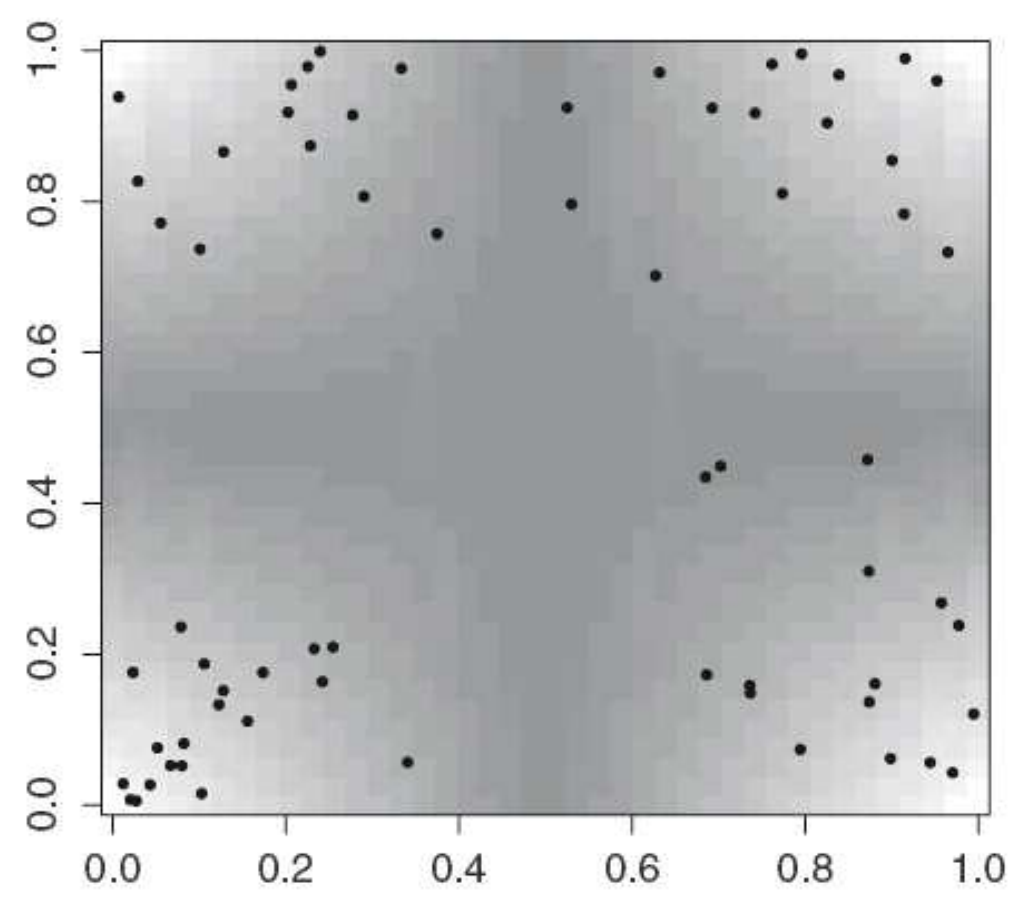

(b) 


\section{Répartition plus régulière : modèle à noyau dur (ou hardcore)}

La règle : interdire les points trop proches

- Exemples :

- répartition spatiale d'animaux (compétition)

- boulangeries dans une ville

- centres de cellules

- arbres dans une forêt (??)

- en physique, centres d'«atomes impénétrables»

- Ces modèles vont être défini par leur densité de Gibbs 


\section{Répartition moins régulière : formation d'agrégats (clusters)}

Exemple : le PP de Neymann - Scott

1. Un processus $P$ « parent »: $P P P$ homogène $\lambda$

2. Chaque parents $P(i)$ engendre des enfants en nombre $N$ et en positions $\mathrm{D}$ centrées autour de $P(i)$, aléatoires, $N$ et $D$ indépendantes

Paramètres : $\lambda$, les lois $N$ et $D$ 


\section{Simulation de PP spatiaux avec stat spat}

- owin : crée la fenêtre d'observation (si nécessaire)

- runifpoint : n points uniformes.

- runifpoint 3 : idem mais dans $\mathrm{R}^{\star \star} 3$ (installer le package scatterplot $3 d$ pour la représentation $3 d$ ).

$>\mathrm{X}=$ runifpoint3(5000)

$>\operatorname{plot}(\mathrm{X})$

- rpoispp : simulation d'un PPP (homogène ou non)

- rNeymanScott : PP de N-S avec agrégat

- rThomas....

- rmh : simulation d'un PP à partir de son modèle de densité (Strauss, noyau dur, etc) 


\section{Ajustement $f$ inpines sur un Neymann - Scott}

- (a) Données réelles : modèle de NS à 3 paramètres $\theta=\left(\lambda, \mu, \sigma^{* *} 2\right)$

1 - parents Poisson $\lambda$

2 - nombre de descendants d'un père Poisson $\mu$

3 - répartition des fils autour d'un père Gaussienne sphérique $\sigma^{* *} 2$ $\rightarrow$ Ajustement par MCO (cf. poly) puis

- (b) Simulation du NS estimé ( $\Delta$ parents et $\bullet$ descendants)
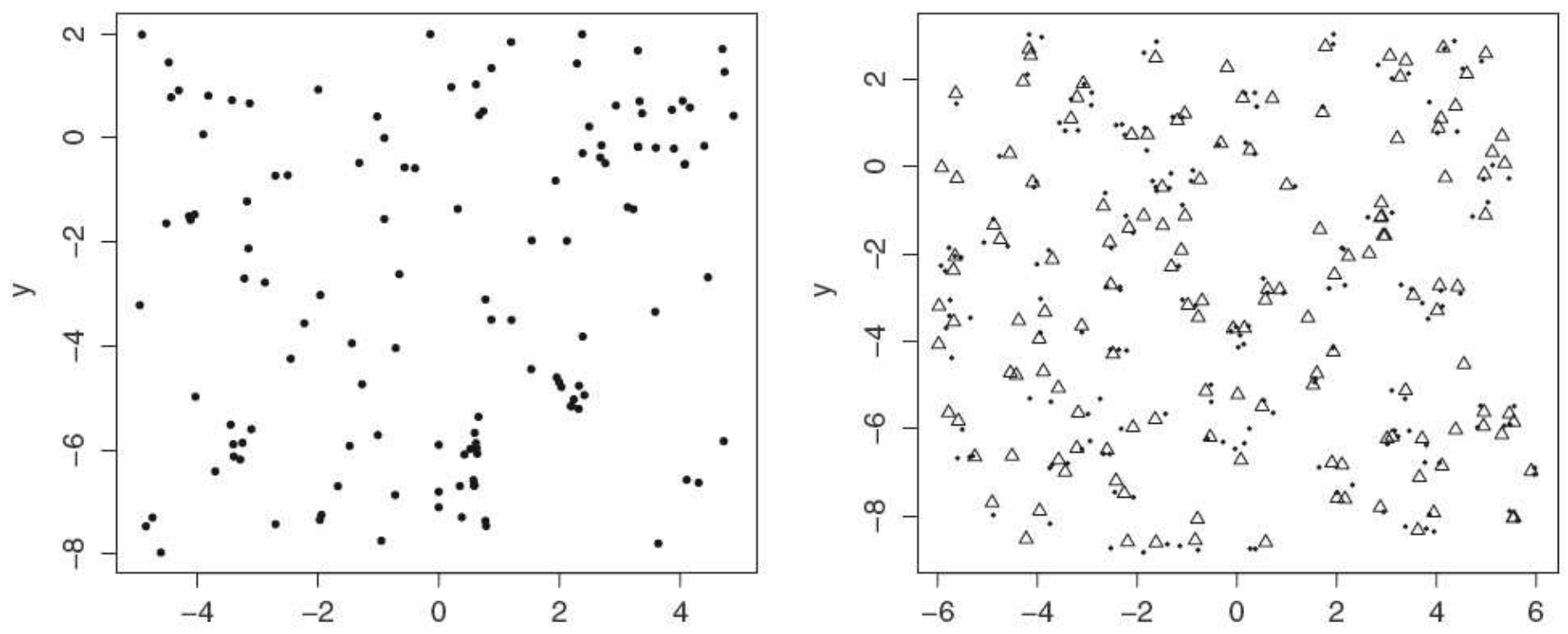

Atelier Spatial RASMA 


\section{PP doublement Poissonien}

- PPP à intensité aléatoire $\{\Lambda(s), s$ dans $S\}$

- Exemple : PP de Cox log-gaussien $X$

- $\wedge$ suit le modèle log-linéaire à effet aléatoire

$$
\log \Lambda(\xi)={ }^{t} z(\xi) \beta+\Psi(\xi) .
$$

- $\psi$ un champ Gaussien centré de covariance $c$

- c contrôle la corrélation spatiale de $X$

(Moller - Waagepertersen) 


\section{Deux exemples de PP de Cox}

- intensité $\Lambda$ en fond grisé $(\Lambda(s)$ grand, fond clair)

- Modèle log-Linéaire : $\beta=z \equiv 1$ partout

- Deux covariances $c$ pour l'intensité aléatoire $\Lambda$

(a) $c(\xi, \eta)=3 \exp \{-\|\xi-\eta\| / 10\} ;$ (b) $c(\xi, \eta)=3 \exp \left\{-\|\xi-\eta\|^{2} / 10\right\}$.
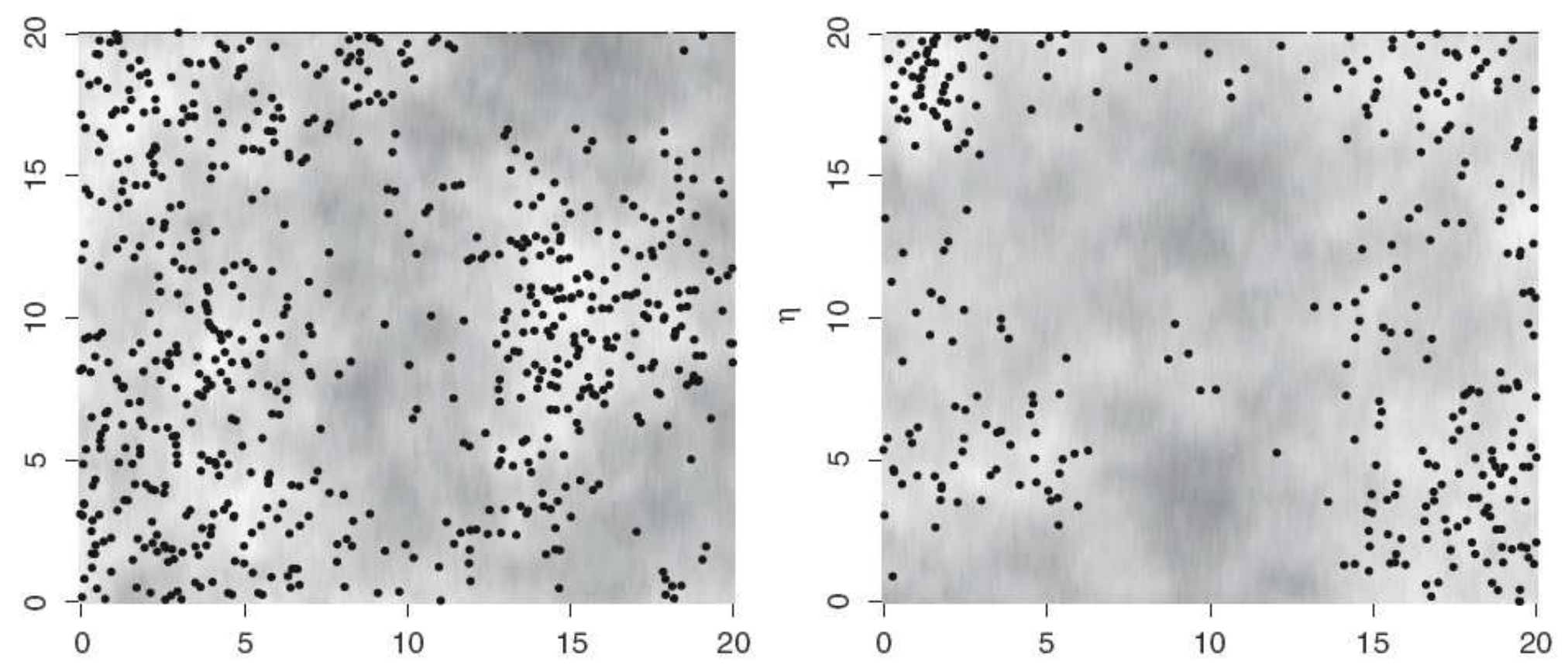

Atelier Spatial RASMA

Saint Louis du Sénégal 


\section{PP marqué (PPM)}

- Une marque $m(x(i))$ s'ajoute en chaque $x(i)$ de $X$

- Exemples :

- marque « disque » centré en x(i) (diamètre arbre)

- rayon $r$ du disque est fixé ou aléatoire $R$

- marques fibres curvilignes attachées à x(i) (système racinaire d'une plante, segment de longueur et orientation aléatoires)

- nombre fini $K$ de marques : $K$ états possibles, une couleur est associée à chaque $x$ (i) (i.e. $K=2$ deux états « sain » ou « malade ») 


\section{Deux exemples de PPM}

(a) Marques binaires : localisation des 62 cas $(\bullet)$ de Leucémie d'un canton et de 141 résidences $(x)$ d'enfants sains $(K=2$, données humberside)

(b) Marques continues : positions et tailles des 584 aiguilles de pin d'un sous bois (données longleaf)

Question sur (a) : effet spatial influençant la maladie?
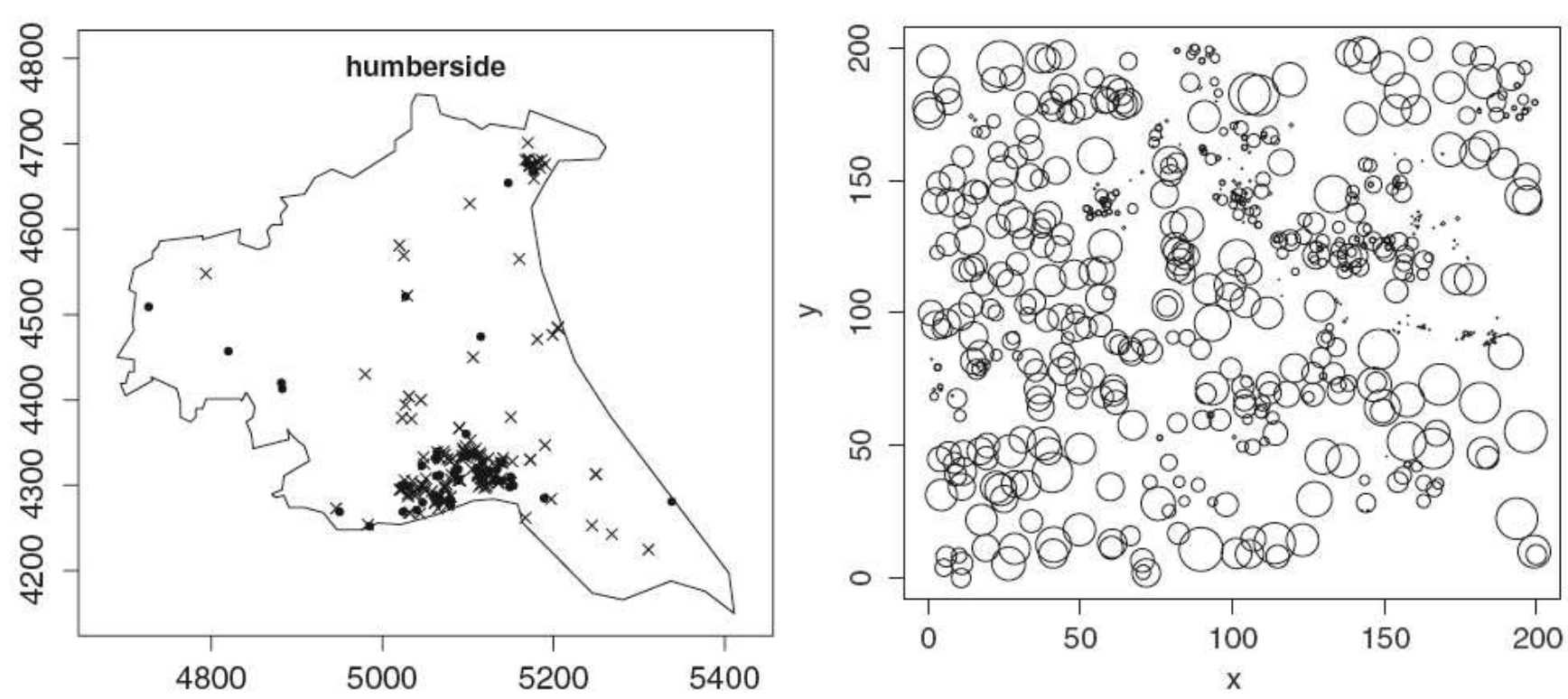

Atelier Spatial RASMA

Saint Louis du Sénégal 


\section{Densité $f$ d'un PP}

- $f$ : densité de probabilité par rapport à un $P P P(1)$

- $f: E \rightarrow R, E=$ espace exponentiel de toute les configurations $x$

- $f$ admissible si intégrable, d'intégrale 1

- En général on définit $f$ à une constante près :

$f(x)=c g(x)$ où $g$ explicite (mais pas $c !$ )

- Inutile connaître c pour la simulation (Metropolis)

- Mais il faut connaître $c=c(\theta)$ pour l'estimation du MV de $\theta$ 


\section{Exemple de PP à densité : PP de Gibbs}

- $U(x) \leftarrow$ potentiel de Gibbs : $U(x)=\Sigma \Phi(A)(x)$

- Admissibilité de $\exp \{U(x)\}$

- Exemple : famille exponentielle

$$
\boldsymbol{f}(x)=c(\theta) \exp \{<\theta, T(x)>\}
$$




\section{PP de Strauss}

- $U(x)$ dérive de 2 statistiques issues de $x$ :

$1-n(x)=$ nombre de points de $x$

$2-s(x)=$ nombre de couples de $x$ à distances $<r$

- Energie : $U(x)=a n(x)+b s(x) \quad($ ou $a=\log \beta$ et $b=\log \gamma)$

$$
f_{\theta}(x)=c(\theta) \beta^{n(x)} \gamma^{s(x)}, \quad \theta={ }^{t}(\beta, \gamma) .
$$

$\beta$ (ou a) règle l'intensité de $x ; y$ règle la régularité spatiale :

$-\gamma<1$ : d'autant plus régulière que $Y$ petit

$-\gamma=1: P P$ de Poisson homogène d'intensité $b=\log (Y)$

$-\gamma>1$ : formation d'agrégats

- $P P$ à noyau dur : $\gamma=0$, interdit les couples à distance $<r$ 


\section{Simulation Metropolis d'un PP de Gibbs}

On circule dans les espaces $E(n)$ en autorisant à une itération :

$\rightarrow$ soit une naissance (proba $1 / 2$ )

$\rightarrow$ soit une mort (proba $1 / 2$ )

suivant la règle suivante :

$$
f_{\theta}(x)=c(\theta) \beta^{n(x)} \gamma^{s(x)}, \quad r(x, x \cup \xi)=\frac{\nu(S) f(x \cup \xi)}{n(x) f(x)}
$$

- Naissance $\xi$ retenue avec la proba $\inf \{1, r(x, x \cup \xi\}$;

- sinon rester en $x$.

- Mort $\eta$ retenue avec la probabilité $\inf \left\{1, r(x \backslash \eta, x)^{-1}\right\}$;

- sinon rester en $x$.

Cet algorithme simule le PP de densité $f$. 


\section{Quelques outils statistiques}

- Moments d'ordre 1 ou intensité

(modèle sur la moyenne)

- Moments d'ordre 2, corrélation repondérée

(indépendance spatiale ou non)

- Moment réduit $K$ d'ordre 2 de Ripley

- Distances aux plus proches voisins 


\section{Moments (intensités) d'ordre 1 et 2 d'un PP}

Moment d'ordre 1

si $B$ borélien borné : $\lambda(B)=E(N(B))$

Intensité $\rho$ d'ordre $1: \lambda(d x)=\rho(x) d x$

Intensité d'ordre 2

$$
\rho_{2}(x, y)=\frac{P(N(d x)=1 \text { et } N(d y)=1)}{d x d y}
$$

Corrélation de paires repondérée

$$
g(x, y)=1+\frac{\operatorname{Cov}(N(d x), N(d y))}{\rho(x) \rho(y) d x d y}
$$

1. $g(\xi, \eta)=1$ si les points apparaissent indépendamment

2. $g(\xi, \eta)>1$ traduit une attraction entre les points (c positive).

3. $g(\xi, \eta)<1$ traduit une répulsion entre les points (c 


\section{Moment K de Ripley (cas isotropique)}

Soit $X$ un PP isotropique de densité $\rho$.

Soit $B(x, h)$ la boule de centre $x$ et de rayon $h$.

Soit $x$ un point de la réalisation $X$. Alors :

$$
\begin{gathered}
\rho \times K(h)=E(\text { nb points } X \text { dans } B(x, h)) \\
K \text { est liée à̀ } \rho_{2}: \text { si } d=2, \rho^{2}(h) K(h)=2 \pi \int_{0}^{h} u \rho_{2}(u) d u
\end{gathered}
$$




\section{K et régularité spatiale?}

Soit $X$ un PP de densité fixé sur $\mathbb{R}^{2}$

et la fonctionnelle $L(h)=\sqrt{\frac{K(h)}{\pi}}$. On a :

$h \mapsto L(h)$ est $\left\{\begin{array}{l}\equiv h \text { si } X \text { est un PP de Poisson } \\ \text { convexe si } X \text { est plus régulier (noyau dur) } \\ \text { concave si } X \text { présente des aggrégats (NS) }\end{array}\right.$ 


\section{Distances aux plus proche voisins (ppv)}

1 - d'un point $\bullet$ de $X$

2 - d'un point o de la fenêtre d'observation

Deux distributions de distance au ppv :

1 - d'un point $x=\bullet$ de $X$ :

$G_{x}(r)=G(r)=P(d(x, X \backslash\{x\}) \leq r) ;$

2 - d'un point $x=\circ$ de $S(x \notin X)$ :

$F(r)=P(d(x, X \backslash\{x\}) \leq r)$. 


\section{Distances aux ppv et régularité spatiale?}

Soit la fonctionnelle associée aux 2 distances:

$$
J(r)=\frac{1-G(r)}{1-F(r)}
$$

Si $X$ est stationnaire :

Alors si $J\left\{\begin{array}{l}=1, X \text { est un } \mathrm{PP} \text { de Poisson } \\ <1 \text { indique plus de régularité (noyau dur) } \\ >1 \text { indique moins de régularité (NS) }\end{array}\right.$

$r \mapsto J(r)$ bonne statistique pour évaluer la dépendance spatiale 


\section{Questions de base pour un PP}

- La répartition spatiale $X$ est-elle due uniquement au hasard ? (CSR = Complete Spatial Randomness) ou non ?

- Si non, y a t’il compétition ? coopération ?

- Quel modèle de PP spatial proposer pour $X$ ?

- Comment estimer le modèle ? Comment le valider ?

$\rightarrow$ Méthodes paramétriques

$\rightarrow$ ou méthodes de Monte Carlo 


\section{Test de «CSR » : X est un PPP}

Utilisation des distances aux PPV et fonctionnelle $J$ Estimation de $J \leftarrow$ estimations empiriques de $G$ et $F$

Indicateur de régularité spatiale : $J(r)=\frac{1-G(r)}{1-F(r)}$,

$\Rightarrow$\begin{tabular}{|c||c|c|c|}
\hline Valeur de $J$ & $J<1$ & $J=1$ & $J>1$ \\
\hline \hline répartition & + régulière & au hasard (PPP) & - régulière \\
\hline
\end{tabular}

$X \sim P P P(\lambda)$ homogène sur $\mathbb{R}^{2}: G(r)=F(r)=1-\exp \left\{-\lambda \pi r^{2}\right\}$. 


\section{Test de « CSR » : utilisation de K}

Autre indicateur : $L(h)=\sqrt{\frac{K(h)}{\pi}}$

$\Rightarrow$\begin{tabular}{|l||l|l|l|}
\hline Type de $L$ & convexe & linéaire & concave \\
\hline \hline répartition & + régulière & au hasard (PPP) & - régulière \\
\hline
\end{tabular}

L'étimation de $L$ découle naturellement de celle de $K$. 


\section{Test de «CSR » : comptages par quadrats}

Chi 2 d'ajustement sur une loi de Poisson ( inpines)

- On forme (par exemple) $4 \times 4=16$ quadrats même surface

- Comptage des effectifs $N(i)$ pour chaque quadrat $i$

- Distribution empirique des $N$ (i) (à droite)

- Distance à une distribution de Poisson : $\mathrm{D}=46.7>>X^{2}(15 ; 5 \%)$
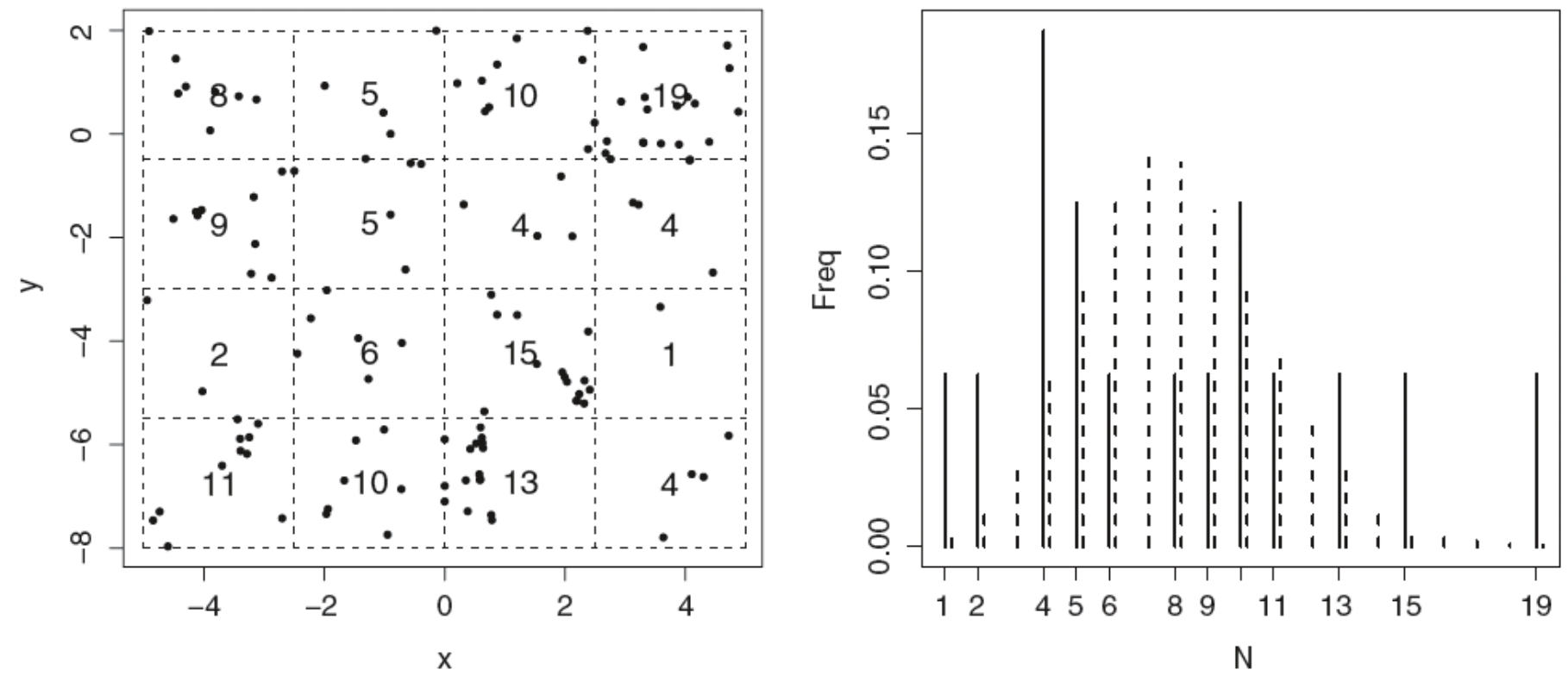


\section{Estimation de l'intensité du PP}

- Méthodes non paramétriques classiques : fenêtrage et noyau de convolution

- Modèle paramétrique : définir le modèle et estimer $\rightarrow$ par MV en supposant CSR (c'est une PV si CSR non vérifiée)

$\rightarrow$ bonne propriétés asymptotiques si X est ergodique 


\section{Estimation NP d'une densité d'un PP}

\section{(a) données érables (Iansing) et}

3 estimations (b-c-d) avec noyaux de + en + régularisant

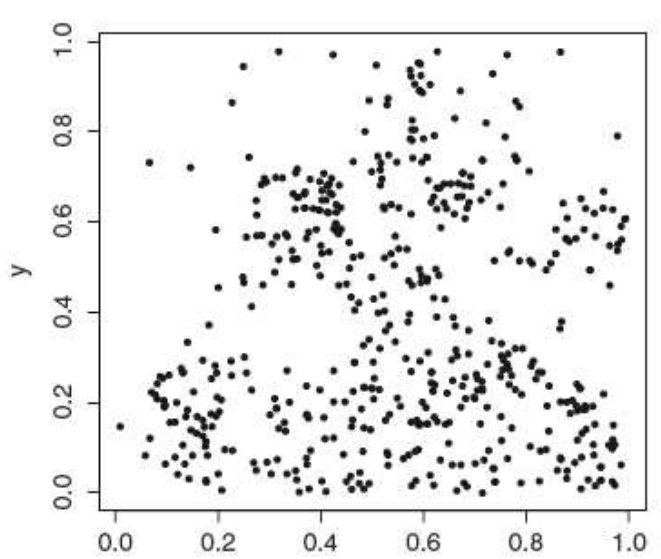

(a)

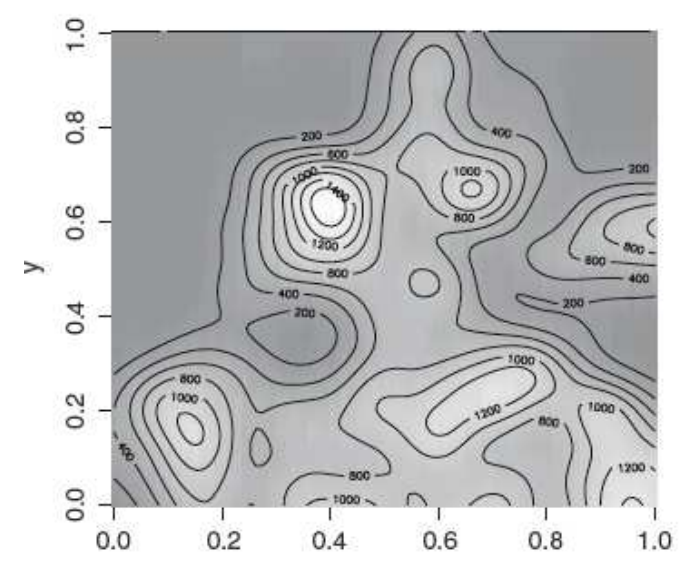

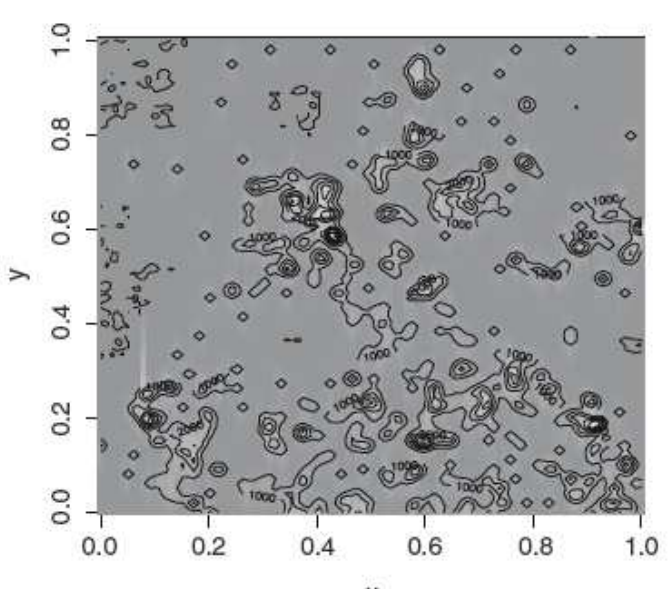

(b)

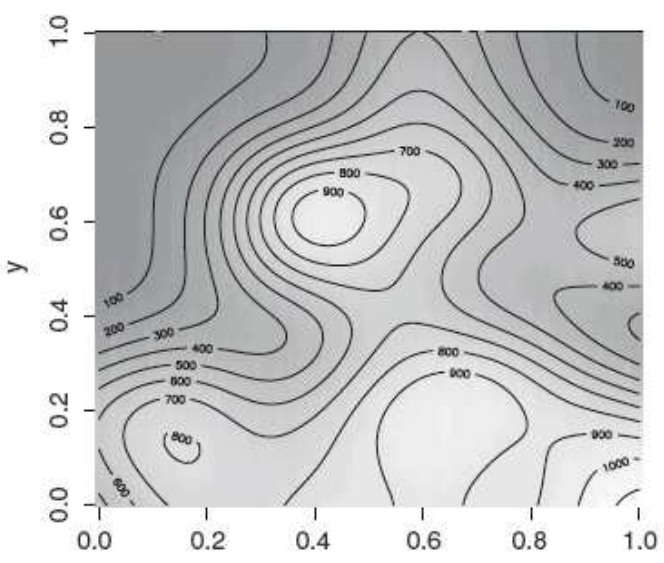

Atelier Spatial RASMA 


\section{Estimations NP des distances aux ppv et $K$}

1 - à partir de statistiques empiriques de comptage

2 -- lissage postérieur éventuel

Moment $\mathrm{K}$ de Ripley : sur le disque $B$ centré en $O$ de rayon $h$ si $X$ est de densité $T$ :

$$
\tau^{2} \widehat{\mathcal{K}}(B)=\frac{1}{\nu(A)} \sum_{\xi, \eta \in X \cap A}^{\neq} 1_{B}(\xi-\eta)
$$

Distribution G aux ppv : $x$ un point de $X, h(i)$ les $n$ distances $d(X, X(i))$ pour les $n$ points de $X$ :

$$
\widehat{G}(h)=\frac{1}{n} \sum_{i=1}^{n} 1_{\left(0, h_{i}\right]}(h)
$$




\section{Estimation d'un modèle d'intensité $\rho(\bullet, \theta)$}

- Si $X$ est PPP d'intensité $\rho(\bullet, \theta)$, la log-vraisemblance de $\{x(1), x(2), \ldots, x(n)\}$ sur $A$ est donnée ci-dessous si $\rho(\bullet, \theta)$ suit un MLG, maximisation via un logiciel dédié

- Si $X$ est $P P$ de densité $\rho(\bullet)$, on maximise encore cette Pseudo-Vraisemblance

Bonne propriété limite si $X$ ergodique et $A \rightarrow R^{* * 2}$.

$$
l_{A}(\theta)=\sum_{\xi \in x \cap A} \log \rho(\xi ; \theta)+\int_{A}\{1-\rho(\eta ; \theta)\} d \eta .
$$




\section{Moindres carrés pour un modèle $K(,, \theta)$}

1 - choix d'une famille $H$ de distances identifiant $\theta \rightarrow K(., \theta)$

2 - MCO sur une puissance $c$ de $K$

Choix (Diggle) : $c=0.5$ si $X$ régulier, 0.25 si $X$ avec agrégats

$$
\begin{aligned}
& D^{*}(\theta)=\sum_{i=1, k} w_{i}\left\{\hat{K}\left(h_{i}\right)^{c}-K\left(h_{i} ; \theta\right)^{c}\right\}^{2} ; \\
& k \text { distances } \mathcal{H}=\left\{h_{1}, h_{2}, \cdots, h_{k}\right\} .
\end{aligned}
$$




\section{Autres méthodes paramétriques}

1 - Pour un PP, on sait définir une densité conditionnelle comme pour un champ de Markov sur un réseau (Jensen-Moller) $\rightarrow$ PVC pour un PP

2 - On maximise cette PVC : sous conditions d'ergodicité et de faible dépendance, « bons résultats asymptotiques

3 - Estimation par MV : la difficulté est le calcul de la constante de normalisation $\rightarrow$ l'approcher par Monte Carlo 


\section{Estimation d'un PP avec spat stat}

Déclarer le modèle paramétrique : Poisson, Strauss, Hardcore, StraussHard, Geyer, N-S, Thomas....

- ppm : ajuste le modèle aux données via la pseudo vraisemblance conditionnelle (si PPP, c'est la vraisemblance); consulter les exemples, i.e :

$>\operatorname{data}$ (nztrees) puis > plot (nztrees)

$>$ ppm(nztrees, $\mathrm{x}$, Strauss (13), correction="periodic")

- rmh : simule le modèle estimé $\rightarrow$ procédure « visuelle » de validation. 


\section{Estimation d'un PP avec spat stat (suite)}

- fitin : donne l'interaction (0 pour un PPP) du PP estimé

- lgcp . estK : ajuste un PP de Cox log-gaussien à covariance exponentielle par MC sur la base de la statistique $\mathrm{K}$

- thomas . est $K$ : idem pour un PP de Thomas

- log . link: donne la log-vraisemblance de l'ajustement pour un PPP; on en déduit le critère AIC.

- density : lissage de densité par convolution (noyau $\mathrm{k}$ à choisir) 


\section{Estimation d'un PP avec spatstat: statistique et bande de confiance $G$, $J$ et $K$}

- Gest : estimation de la densité cumulée de la distance d'un point de la configuration $X$ à son $p p v$ dans $X$ ( $F$ si distance d'un point courrant à son ppv dans $X)$

- Jest : $J(r)=(1-G(r)) /(1-F(r))$. $\mathrm{J}>1,=1$ et $<1$ indique plus régulier, Poisson, moins régulier

- Kest : estime le moment d'ordre $2 \mathrm{~K}$ de Ripley.

- Kinhom : pour un PP inhomogène

- envelope : calcule les bandes de confiances par simulation des statistiques de base $(K, G, J \ldots)$

- Quadrat . test : test du chi2 de l'hypothèse CSR d'indépendance spatiale. 


\section{Test de Monte Carlo de $\mathrm{H}(0)$ : « X est CSR »}

\section{Test basé sur un intervalle de confiance sur $\mathrm{K}$}

On observe $x$. Choisir $L$ distances $\{h(1), h(2), h(L)\}$.

- Estimer (empiriquement) $K$ par $K^{*}(h(l))$ à ces $L$ distances

- Sous $\mathrm{H}(0)$, estimation $\rho^{*}$ de l'intensité $\rho$ d'un PPP

- Simulation de $m$ réalisations $x^{*}(I), l=1, m$, d'un $\operatorname{PPP}\left(\rho^{*}\right)$ (i.e. $m=20$ )

- $\rightarrow m$ estimations $K^{*}(i, h(I))$ associées à chaque $x^{*}(i)$

- Enveloppes inf et sup de ces $m$ estimations

- Si $K^{*}$ se trouve entre ces 2 enveloppes, accepter $\mathrm{H}(0)$ (ici, niveau 10\%)

On peut aussi comparer les $K^{*}$ à $\pi h^{2}$, valeur théorique de $K$ pour un PPP. Cette méthode décrit le principe du Bootstrap paramétrique. 


\section{Les 156 pins d'une forêt finlandaise}

(finpines)

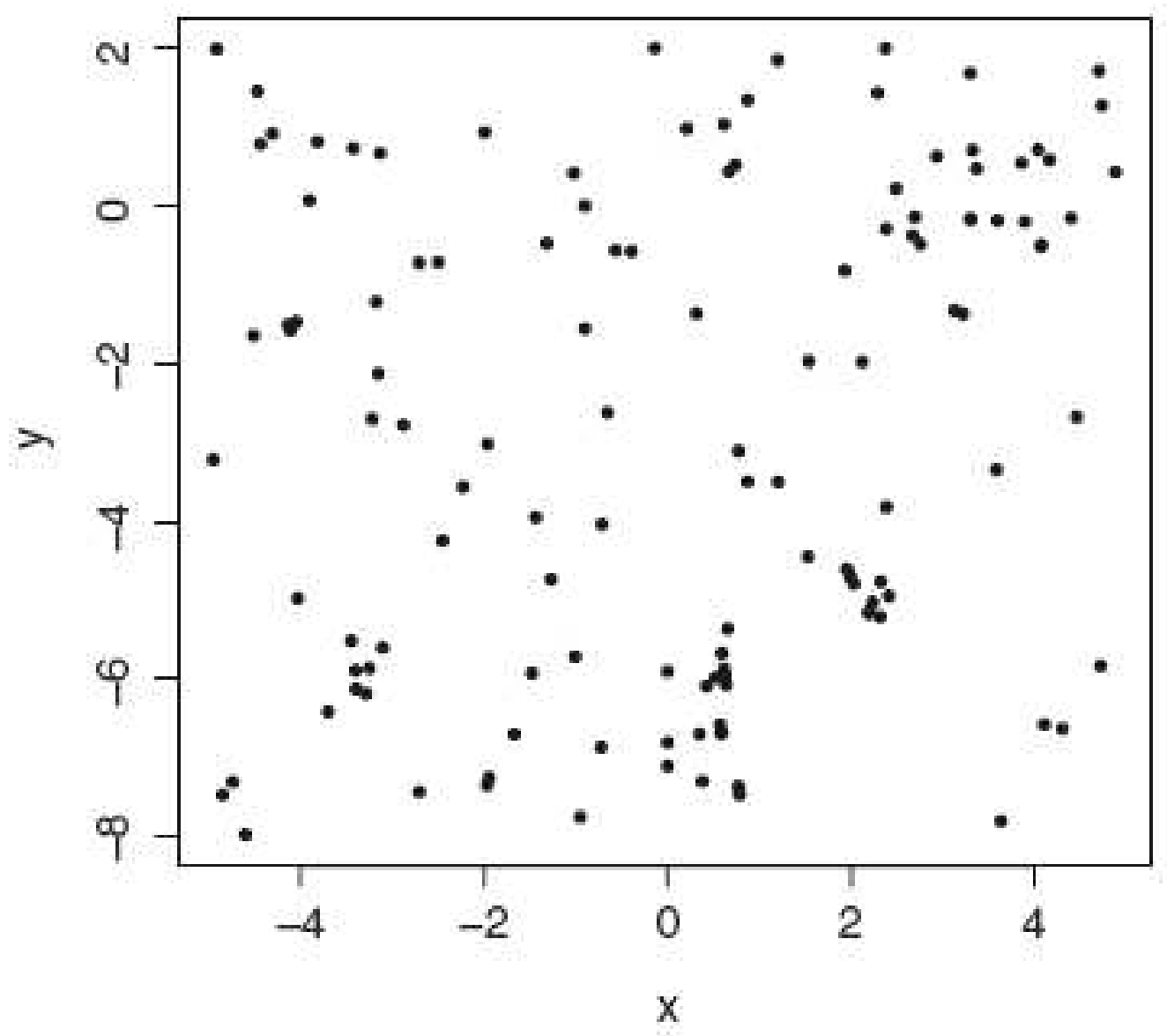




\section{«Indépendance $H(0)$ » contre « Neyman-Scott »}

(données finpines)

- En continu, le graphe estimé de $h \rightarrow L(h)-h(=0$ si $(H(0))$ pour $x$

- À gauche : bande de confiance pour $m=40$ simulations $x^{*}$ sous $\mathrm{H}(0)$

- À droite : bande sous l'alternative $\mathrm{N}-\mathrm{S}$, avec en pointillé la courbe $h \rightarrow K(h)$ théorique pour les paramètres de $\mathrm{N}-\mathrm{S}$ estimés.
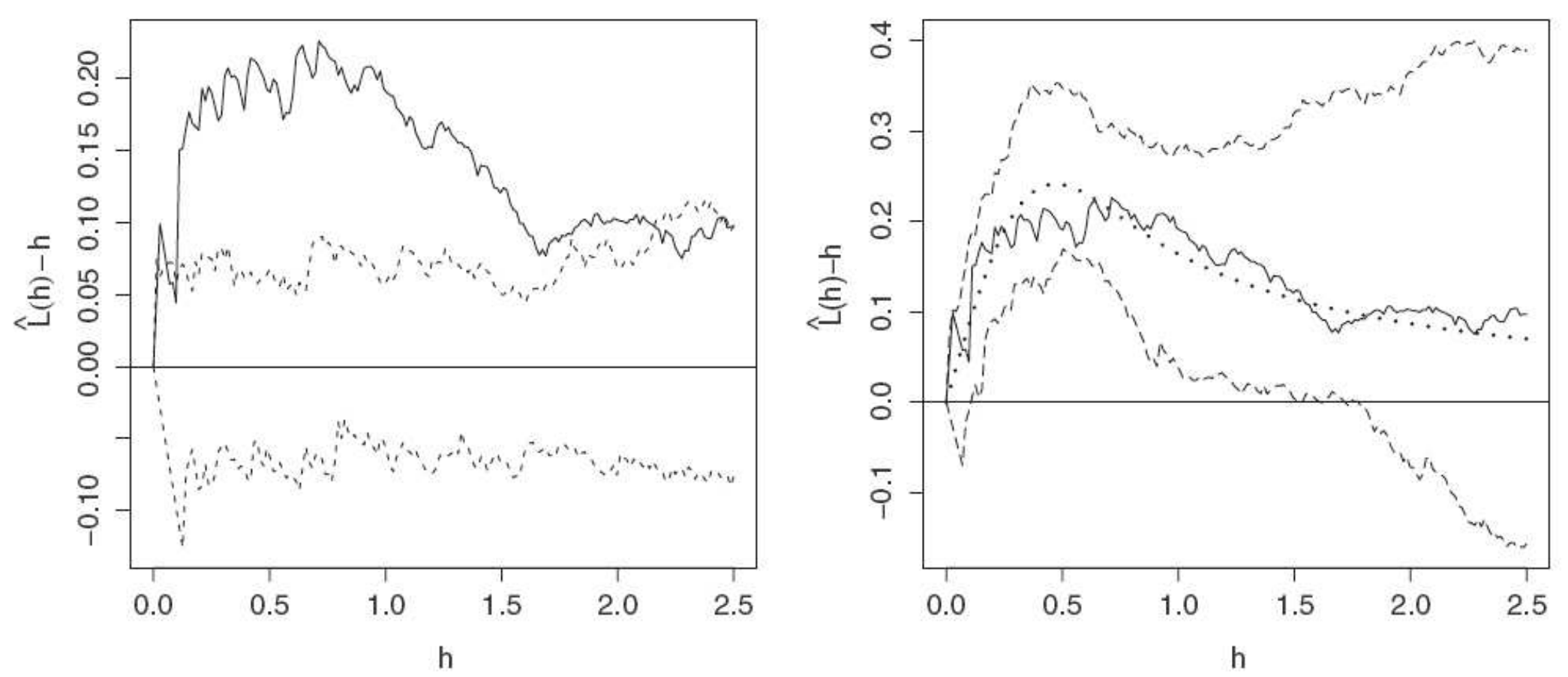

Atelier Spatial RASMA 


\section{spatstat: le package $\boldsymbol{R}$ pour les PP spatiaux}

(spatial PP analysis, model fitting, simulation, ...)

- Anova.ppm: déviance (s) entre deux ou plus que deux modèles

- Jest : estimation (empirique) de l'indicateur J du caractère (J=1) PPP ou non

- Kest : idem pour la fonction moment réduit d'ordre $2, \mathrm{~K}$

- nearest.neighbour: distances aux ppv
- Ppm : ajuste un model de PP à des données,

- $\boldsymbol{m}$ h.ppm : simulation par Metropolis-Hastings ( $\mathrm{mh}$ ) d'un modèle de PP,

- rpoispp : simulation d'un PPP hogène ou non)

- Données : cells, finpines, 